\title{
First order analytic difference equations and integrable quantum systems
}

S. N. M. Ruijsenaars

Centre for Mathematics and Computer Science, P.O. Box 4079,

1009 AB Amsterdam, The Netherlands

(Received 3 January 1996; accepted for publication 26 July 1996)

We present a new solution method for a class of first order analytic difference equations. The method yields explicit "minimal" solutions that are essentially unique. Special difference equations give rise to minimal solutions that may be viewed as generalized gamma functions of hyperbolic, trigonometric and elliptic type-Euler's gamma function being of rational type. We study these generalized gamma functions in considerable detail. The scattering and weight functions $(u$ and $w$-functions) associated to various integrable quantum systems can be expressed in terms of our generalized gamma functions. We obtain detailed information on these $u$ - and $w$-functions, exploiting the difference equations they satisfy.

(C) 1997 American Institute of Physics. [S0022-2488(97)01701-5]

\section{TABLE OF CONTENTS}

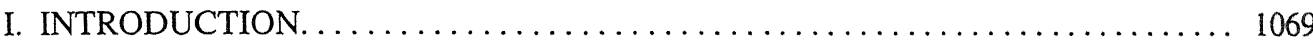

II. GENERAL RESULTS ON ANALYTIC DIFFERENCE EQUATIONS. . .......... 1076

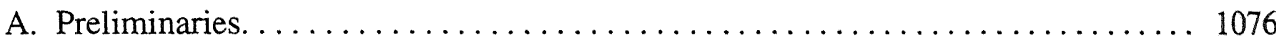

B. Fourier Transform Solutions. . . . . . . . . . . . . . . . . . . . . . . . 1079

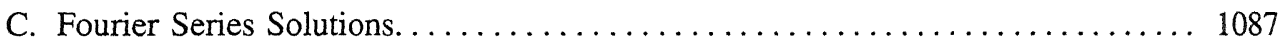

III. GENERALIZED GAMMA FUNCTIONS. . . . . . . . . . . . . . . . . . . . . 1091

A. The Hyperbolic Case. . . . . . . . . . . . . . . . . . . . . . . . . . . . . . . . . . . . . 1091

B. The Elliptic Case. . . . . . . . . . . . . . . . . . . . . . . . . . . . 1101

C. The Trigonometric Case .................................. 1107

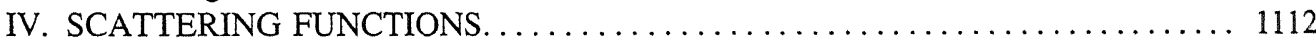

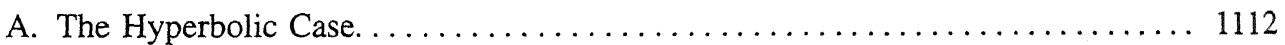

B. The Elliptic Case . . . . . . . . . . . . . . . . . . . . . . . . . 1121

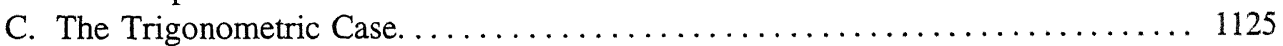

V. WEIGHT FUNCTIONS. . . . . . . . . . . . . . . . . . . . . . . . 1128

A. The Hyperbolic Case . . . . . . . . . . . . . . . . . . . . . . 1128

B. The Elliptic Case. . . . . . . . . . . . . . . . . . . . . . . . . 1132

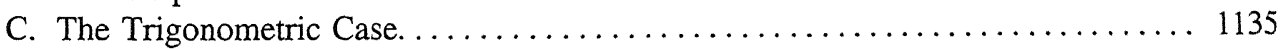

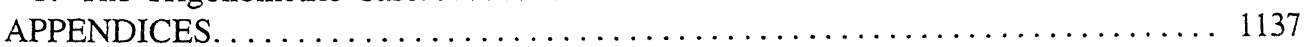

A. THE GAMMA FUNCTION AND RELATED INTEGRALS. . . . . . . . . . 1137

B. UNIFORM ESTIMATES. . . . . . . . . . . . . . . . . . . . . . . . . 1142

\section{INTRODUCTION}

This paper is concerned both with the general theory of first order analytic difference equations (from now on $\mathrm{A} \Delta \mathrm{Es}$ ) and with certain special functions that arise as solutions to $\mathrm{A} \Delta \mathrm{Es}$ of a quite restricted type. As announced and partly detailed in our survey ${ }^{1}$ and lectures, ${ }^{2}$ among these special functions are the weight functions and scattering amplitudes associated with relativistic quantum integrable systems of Calogero-Moser type-which, in turn, for special parameter 
choices reduce to functions occurring in various well-known infinite-dimensional integrable systems, such as the sine-Gordon theory, the XYZ chain and the eight-vertex model.

The first part of the paper (Sections II and III) does not involve integrable systems. To describe the scope of the results obtained therein, we start from two quite elementary first order $\mathrm{A} \Delta \mathrm{Es}$, namely,

$$
\begin{gathered}
M(w+1)=c M(w), \quad w \in \mathbb{C}, \quad c \in \mathbb{C}^{*}, \\
M(w+1)=w M(w), \quad w \in \mathbb{C} .
\end{gathered}
$$

Obviously, the first one is solved by the function $\exp (w \ln c)$ and the second one by Euler's gamma function $\Gamma(w)$. These functions can be used as building blocks for solving $\mathrm{A} \Delta \mathrm{Es}$ of the form

$$
M(w+1)=Q(w) M(w), \quad w \in \mathbb{C},
$$

where $Q(w)$ is a rational function of $w$. Indeed, any function of the form

$$
M(w)=e^{a w} \frac{\prod_{j=1}^{M} \Gamma\left(w-b_{j}\right)}{\prod_{k=1}^{N} \Gamma\left(w-c_{k}\right)}, \quad a, b_{j}, c_{k} \in \mathbb{C},
$$

satisfies (1.3) with $Q(w)$ rational, and varying the parameters $a, M, N, b_{j}, c_{k}$, yields all rational functions.

Suppose now that one can find meromorphic solutions to the $\mathrm{A} \Delta \mathrm{E}(1.3)$ for $Q(w)$ equal to the Weierstrass $\sigma$-function $\sigma\left(w ; \omega, \omega^{\prime}\right)$ with $\omega,-i \omega^{\prime} \in(0, \infty)$, and its trigonometric $\left(-i \omega^{\prime}=\infty\right)$ and hyperbolic $(\omega=\infty)$ degenerations-the sine and sinh-functions. (The additional factor $c \exp \left(a w^{2}\right)$ in the degenerate $\sigma$-functions is easily taken into account-one need only include a factor $\exp (P(w))$ with $P(w)$ a third order polynomial.) Then the respective solutions $M_{\text {ell }}(w), M_{\text {trig }}(w)$ and $M_{\text {hyp }}(w)$ can be used as building blocks to solve the A $\Delta \mathrm{E}$ (1.3) with $Q(w)$ any elliptic function with periods $2 \omega, 2 \omega^{\prime}$ or its trigonometric and hyperbolic counterparts, resp. Indeed, any elliptic function $Q(w)$ can be written in the form (1.4), with the exponential replaced by a constant and $\Gamma(w)$ by $\sigma(w)$, so a corresponding meromorphic solution $M(w)$ to (1.3) is obtained by taking $\Gamma \rightarrow M_{\text {ell }}$ in (1.4).

Among other things, this paper presents and studies special functions generalizing the gamma function, which can be used as building blocks to solve $A \Delta E$ s of the three types just described. In one case the pertinent function is not really new-up to a constant and an exponential it amounts to Thomae's q-gamma function. ${ }^{3,4}$ For the other two cases, however, the corresponding generalized gamma functions are new, and turn out to have some quite remarkable properties. The comprehensive study of these functions (to be found in Section III) constitutes one of the principal results of this paper.

In order to sketch the setting from which our generalized gamma functions emerge, we begin by pointing out that even when one restricts attention to functions $Q(w)$ and solutions $M(w)$ that are meromorphic (as we do), there is an enormous ambiguity in the solution. Indeed, assuming $M(w)$ is a solution and $m(w)$ any meromorphic function with period 1 , it is obvious that the function $m(w) M(w)$ is a solution as well. The importance of singling out solutions with special properties is therefore evident.

In previous literature, the class of $\mathrm{A} \Delta \mathrm{Es}$ to be studied-that is, the class of meromorphic functions $Q(w)$-has been narrowed down by insisting that $Q(w)$ have a special asymptotics for $\operatorname{Re} w \rightarrow \infty$. In particular, Nörlund in his comprehensive monograph ${ }^{5}$ uses this prescribed asymptotics to construct the uniquely determined solution he refers to as the "Hauptlösung" (see also Refs. 6-8). 
By contrast, the key requirement on $Q(w)$ and $M(w)$ we impose is a special asymptotics for $|\operatorname{Im} w| \rightarrow \infty$, satisfied in particular for functions $Q(w)$ that are periodic in the imaginary direction. As will transpire below, this leads to essentially the same solutions only for rational and hyperbolic $Q(w)$, whereas Nörlund's methods do not apply to the trigonometric and elliptic cases.

As a matter of fact, we have opted for a shift in the imaginary direction-in contrast to the shift by 1 in the $A \Delta E$ (1.3). This corresponds to the applications to integrable systems, and is also convenient in view of our different requirements concerning asymptotics. Moreover, we shall treat the step size as a variable, and we do not single out the positive or negative imaginary direction. Thus our starting point is the $\mathrm{A} \Delta \mathrm{E}$

$$
F(z+i a / 2)=\Phi(z) F(z-i a / 2)
$$

where $\Phi(z)$ is meromorphic, and where the step size $a$ is an arbitrary positive number. Of course, this $\mathrm{A} \Delta \mathrm{E}$ is related by a scaling and a shift over half the step size to the $\mathrm{A} \Delta \mathrm{E}(1.3)$, so all results can be rephrased for (1.3)-at the expense, however, of cumbersome notation, which moreover hides some symmetries that naturally emerge when the second convention is used.

We are now prepared to describe the organization and results of the paper in more detail. Section II contains our general results on first order A $\Delta$ Es. In Subsection II A we set the stage by delineating the class of functions $\Phi(z)$ allowed in (1.5). As a first requirement, we insist on $\Phi(z)$ being free of zeros and poles in a strip $|\operatorname{Im} z|<s, s>0$. We denote such A $\Delta$ Es as regular $\mathrm{A} \Delta \mathrm{Es}$, and refer to solutions that are free of zeros and poles in the strip $|\operatorname{Im} z|<s+a / 2$ as regular solutions. The poles and zeros of a regular solution $F(z)$ outside $|\operatorname{Im} z|<s+a / 2$ are completely determined by the poles and zeros of $\Phi(z)$ outside $|\operatorname{Im} z|<s$, as easily follows from (1.5).

Regular $\mathrm{A} \Delta \mathrm{Es}$ can be rewritten in the additive form

$$
f(z+i a / 2)-f(z-i a / 2)=\phi(z), \quad|\operatorname{Im} z|<s,
$$

where $\phi(z)$ denotes (a suitable branch of) $\ln \Phi(z)$. Thus the search for regular solutions to (1.5) is reduced to finding solutions $f(z)$ to (1.6) that are analytic for $|\operatorname{Im} z|<s+a / 2$. Using well-known properties of the partial differential operator $\partial / \partial \bar{z}=\partial_{x}+i \partial_{y}$ and Runge's approximation theorem, it can be proved that such solutions exist. We shall not detail this, however, since the existence arguments yield no information on the solution thus obtained. (An existence proof can be assembled from Ref. 9, for example.)

By contrast, the extra requirements we impose on $\Phi(z)$ (or equivalently $\phi(z)$ ) enable us to construct explicit solutions, with special properties that render them essentially unique. Roughly speaking, we require that $\phi(z)$ have at worst polynomial increase as $|\operatorname{Re} z| \rightarrow \infty$, and construct solutions $f(z)$ with the same property, which are moreover regular (i.e., analytic for $|\operatorname{Im} z|<s+a / 2)$. We refer to such solutions as minimal solutions: both their singularities and their asymptotics for $|\operatorname{Re} z| \rightarrow \infty$ are "best possible" - being enforced by the singularities and asymptotics of $\phi(z)$. Among other things, Theorem II.1 entails the uniqueness up to a constant of minimal solutions to the additive $\mathrm{A} \Delta \mathrm{E}$ (1.6)-assuming they exist.

In Subsections II B and II C we study two classes of $A \Delta E s$ that do admit minimal solutions-as is shown by exhibiting a minimal solution via explicit formulas involving $\phi(x), x$ $\in \mathbb{R}$. The key results are Theorem II.2 and II.5, resp. Theorem II.2 presupposes that $\phi(x)$ is an $L^{1}(\mathbb{R})$-function, whose Fourier transform $\hat{\phi}(y)$ is in $L^{1}(\mathbb{R})$, too, and satisfies $\hat{\phi}(y)=O(y)$ for $y \rightarrow 0$; its corollary Theorem II. 3 handles functions that have these properties after taking a certain number of $x$-derivatives. In Theorem II.5 it is assumed that $\phi(x)$ has period $\pi / r, r>0$, and its zeroth Fourier coefficient vanishes; then Theorem II.6 handles functions $\phi(x)$ for which $\phi^{(k)}$ $(x), k \in \mathbb{N}^{*}$, has these properties.

The arbitrary additive constant in a minimal solution to the $\mathrm{A} \Delta \mathrm{E}$ (1.6) can and will be fixed in the Fourier transform setting of Theorem II. 2 by requiring that the solution go to 0 for $x$ to $\infty$; in the Fourier series setting of Theorem II.5 it is fixed by requiring that the minimal solution 
(which is shown to be $\pi / r$-periodic) have vanishing zeroth Fourier coefficient. The unique solution $f(a ; z)$ thus obtained is given by (2.26) and (2.106), resp. From the identity (2.38) it then follows that $f(a ; z)$ satisfies the addition formula (2.28) in both settings.

The solution $f(a ; z)$ has another illuminating feature: In both cases it satisfies

$$
\lim _{a \backslash 0} \operatorname{iaf}(a ; z)=\psi(z), \quad|\operatorname{Im} z|<s,
$$

where $\psi(z)$ is a primitive of $\phi(z)$. Therefore, iaf $(a ; z)$ may be viewed as a "generalized primitive" of $\phi(z)$. It should be noted that this feature is obviously compatible with the $A \Delta \mathrm{E}(1.6)$, but not $a$ priori implied by it: In view of the huge multiplier ambiguity already discussed, the pertinent limit typically does not exist for more general solutions.

Theorems II.4 and II.7 are concerned with the $a \downarrow 0$ limit of minimal solutions to the $\mathrm{A} \Delta \mathrm{E}$ (1.6) when $\phi$ is allowed to have a suitable $a$-dependence. At first sight, the assumptions may appear very restrictive, but they can in fact be verified for the applications occurring in Section III. The limit (1.7) may be viewed as a quite special consequence of these zero step size limit theorems.

In Appendix A we derive various results that involve Euler's gamma function, not only as a concrete illustration of the theory developed in Subsections II A and II B, but also to prepare the ground for Section III, which is devoted to a study of generalized gamma functions. Below (1.4) we have already delineated the three cases that will be considered in Section III. Since we employ the $\mathrm{A} \Delta \mathrm{E}$ (1.5) and not the $\mathrm{A} \Delta \mathrm{E}$ (1.3), however, the trigonometric case turns into the hyperbolic case and vice versa. Moreover, the Weierstrass $\sigma$-function and its degenerations are traded for close relatives, to which the theory of Section II applies. The resulting minimal solutions (rendered unique in obvious ways) will be dubbed $G$-functions.

More specifically, Subsection III A deals with the hyperbolic $G$-function-the unique minimal solution to the $\mathrm{A} \Delta \mathrm{E}$

$$
G(z+i a / 2)=2 \operatorname{ch}(\pi z / b) G(z-i a / 2), \quad b>0,
$$

that satisfies $G(0)=1$ and $|G(x)|=1$ for real $x$. Now it is evident that any solution $G(z)$ to $(1.8)$ has the property that the quotient $G(z+i b / 2) / G(z-i b / 2)$ is an $i a$-antiperiodic function. It is not at all obvious, though, that a solution exists for which this quotient equals $2 \operatorname{ch}(\pi z / a)$. The hyperbolic $G$-function does have this striking property: It is given by

$$
G_{\text {hyp }}(a, b ; z)=\exp \left(i \int_{0}^{\infty} \frac{d y}{y}\left(\frac{\sin 2 y z}{2 \operatorname{sh} a y \operatorname{sh} b y}-\frac{z}{a b y}\right)\right), \quad|\operatorname{Im} 2 z|<a+b,
$$

and hence is manifestly symmetric under $a \leftrightarrow b$.

We present our results on the hyperbolic $G$-function in seven propositions. Proposition III.1 deals with the three elementary A $\Delta$ Es to which $G$ is a minimal solution, and Prop. III.2 details various automorphy properties. As already noted above, the poles and zeros of a regular solution to (1.5) readily follow from those of $\Phi(z)$; similarly, residues at simple poles can be determined in terms of $\Phi(z)$. This is worked out for $G_{\text {hyp }}$ in Prop. III.3. An important dichotomy first emerges here: When $a / b$ is an irrational number, all poles and zeros are simple, whereas for rational $a / b$ this is not the case.

Since $G_{\text {hyp }}(z)$ is a minimal solution, its logarithm is polynomially bounded for $|\operatorname{Re} z| \rightarrow \infty$ and $|\operatorname{Im} z| \leqslant a / 2$. For the case at hand, the precise asymptotics can be explicitly determined by comparison to the case $a=b$. (This case has special features that render it more accessible.) Proposition III.4 presents the details; the restriction on $|\operatorname{Im} z|$ is readily lifted by exploiting the $\mathrm{A} \Delta \mathrm{E}(1.8)$.

From the representation (1.9) it is already clear that for fixed $z$ in the strip $|\operatorname{Im} 2 z|<a+b$ the $G$-function is real-analytic on $(0, \infty)$ in the parameters $a$ and $b$. In Prop. III.5 we prove that $G$ 
actually extends to a function that is meromorphic in $a, b$ and $z$, as long as the quotient $b / a$ stays away from the negative real axis. This readily follows from a representation for the $G$-function in terms of an infinite product of gamma functions. To control the convergence of this product, some estimates on Laplace transforms assembled in Appendix B are crucial.

The latter estimates are also exploited in proving that a renormalized version of the hyperbolic $G$-function converges to the gamma function when one takes $a=1$ and $|b| \rightarrow 0$ in any sector $|\operatorname{Arg} b| \leqslant \chi, \chi \in[0, \pi)$. This is detailed in Prop. III.6. Two more zero step size limits are obtained in Prop. III.7. In the latter context the limit has branch cuts on the imaginary axis that arise from a confluence of zeros and poles.

Before turning to a sketch of Subsection III B, we would like to mention that $G_{\text {hyp }}$ is not only the key building block for the hyperbolic scattering and weight functions of Subsections IVA and VA, but also for our recent generalization of Gauss' hypergeometric function ${ }_{2} F_{1}$. In this context $G_{\text {hyp }}$ plays the role of the gamma function in the Barnes representation for ${ }_{2} F_{1}$-except that the generalization is far more symmetric. For ${ }_{2} F_{1}$ the symmetry is broken, since a step size is taken to zero that leads to the two quite different limiting functions of Propositions III.6 and III.7 (cf. Ref. 2, Subsection 6.3, and papers to appear).

In Subsection III B we study the elliptic $G$-function, which is given by

$$
G_{\mathrm{ell}}(r, a, b ; z)=\exp \left(i \sum_{n=1}^{\infty} \frac{\sin 2 n r z}{2 n \operatorname{sh} n r a \operatorname{sh} n r b}\right), \quad|\operatorname{Im} 2 z|<a+b
$$

along the same lines as its hyperbolic counterpart (1.9). It is not obvious, but true that $G_{\text {ell }}$ is a minimal solution to an $\mathrm{A} \Delta \mathrm{E}$ of the form

$$
\frac{G(z+i a / 2)}{G(z-i a / 2)}=\exp \left(c_{0}+c_{1} z+c_{2} z^{2}\right) \sigma(z+i b / 2 ; \pi / 2 r, i b / 2)
$$

where $\sigma$ denotes the Weierstrass $\sigma$-function. Thus it can be used as a building block to solve the $\mathrm{A} \Delta \mathrm{E}(1.5)$ with $\Phi(z)$ an elliptic function-as already discussed above.

As it turns out, it is quite convenient to trade the $\sigma$-function $\sigma(z ; \pi / 2 r, i a / 2)$ for a closely related function $s(r, a ; z)(2.89)$. The latter function is odd and $\pi / r$-antiperiodic in $z$, and has limits $r^{-1} \sin r z$ and $\pi a^{-1} \operatorname{sh} \pi a^{-1} z$ for $a \uparrow \infty$ and $r \downarrow 0$, cf. (2.90) and (2.92), resp. Similarly, the function arising on the rhs of (1.11) will be denoted $R(r, b ; z)$. In view of (1.10) it is given explicitly by

$$
R(r, b ; z)=\exp \left(-\sum_{n=1}^{\infty} \frac{\cos 2 n r z}{n \operatorname{sh} n r b}\right), \quad|\operatorname{Im} 2 z|<b,
$$

so it is even and $\pi / r$-periodic in $z$. Most of the propositions in Subsection III B may be viewed as generalizations of hyperbolic counterparts, since one has

$$
\lim _{r \downarrow 0} \exp \left(\pi^{2} / 6 r b\right) R(r, b ; z)=2 \operatorname{ch}(\pi z / b)
$$

and

$$
\lim _{r \downarrow 0} \exp \left(\pi^{2} z / 6 i r a b\right) G_{\mathrm{ell}}(r, a, b ; z)=G_{\mathrm{hyp}}(a, b ; z),
$$

cf. Prop. III.12.

Subsection III C concerns the trigonometric case. Our trigonometric $G$-function is given by 


$$
G_{\text {trig }}(r, a ; z)=\exp \left(\sum_{n=1}^{\infty} \frac{e^{2 i n r z}}{2 n \operatorname{sh} n r a}\right), \quad \operatorname{Im} 2 z>-a,
$$

and can be obtained as a limit of the elliptic $G$-function, viz.,

$$
G_{\text {trig }}(r, a ; z)=\lim _{b \uparrow \infty} G_{\text {ell }}(r, a, b ; z-i b / 2) .
$$

In this case the elementary $\mathrm{A} \Delta \mathrm{E}$ satisfied by the $G$-function reads

$$
\frac{G(z+i a / 2)}{G(z-i a / 2)}=1-e^{2 i r z}
$$

Since the rhs has zeros on the real axis, this is not a regular $\mathrm{A} \Delta \mathrm{E}$. However, any shift $z \rightarrow z+i p, p>0$, yields a regular $\mathrm{A} \Delta \mathrm{E}$, to which the (shifted) $G$-function is a minimal solution.

Propositions III.14-III.19 concern various properties of the $G$-function that are quite easily obtained from the series representation (1.15) or the product representation

$$
G_{\text {trig }}(r, a ; z)=\prod_{m=1}^{\infty} \frac{1}{1-\exp (2 i r z-(2 m-1) a r)} .
$$

Proposition III.20, however, involves more work. Here, we prove that a renormalized version of $G_{\text {trig }}$ converges to the gamma function for $r \downarrow 0$.

Fixing $a>0$, it is clear from (1.18) that $G_{\text {trig }}(r, a ; z)$ extends to a meromorphic function of $r$ and $z$, as long as $r$ stays in the right half plane. But one cannot solve the hyperbolic $\mathrm{A} \Delta \mathrm{E}$, obtained from (1.17) upon taking $r \rightarrow i \pi / b, b>0$, by making use of the trigonometric $G$-function. By contrast, one is allowed to take $b \rightarrow i \pi / r, r>0$, in the hyperbolic $G$-function, yielding the trigonometric function $2 \cos r z$ on the rhs of (1.8). Accordingly, the quotient of the renormalized versions of $G_{\text {hyp }}(1, i \pi / r ; z)$ and $G_{\text {trig }}(r, 1 ; z)$ (both of which converge to the gamma function as $r \downarrow 0)$ is a quite nontrivial $i$-periodic function, cf. (3.171)-(3.173).

Just as in Subsections III A and III B, the last proposition of Subsection III C deals with two zero step size limits; once again, a confluence of zeros and poles gives rise to branch cuts. The subsection is concluded by detailing the relation of our trigonometric $G$-function to the $q$-gamma function.

We continue by sketching the physical setting in which the scattering and weight functions $u(z)$ and $w(z)$ of Sections IV and V, resp., arise. These functions are associated to relativistically invariant integrable generalizations ${ }^{10,11}$ of the nonrelativistic Calogero-Moser $N$-particle quantum systems. ${ }^{12}$ The dynamics of these relativistic systems belongs to a commutative algebra generated by $N$ independent commuting analytic difference operators. The step size in these difference operators is inversely proportional to the speed of light $c$, and for $c \rightarrow \infty$ the commuting difference operators converge to commuting differential operators.

Now a factorized product of $u$-functions is expected to encode the asymptotics of the diagonalizing joint eigenfunction transform, whereas a factorized product of $w$-functions can be used to transform the difference operators and eigenfunctions to an especially convenient form. In particular, in the trigonometric case the transformed eigenfunctions amount to Macdonald's $q$-Jacobi multivariable $A_{N-1}$ polynomials, and the product of weight functions yields the function with respect to which the polynomials are orthogonal (cf. Ref. 2, Subsection 6.2 and references given there). (This is why $w(z)$ is referred to as a "weight function.")

The key point is now that $u(z)$ and $w(z)$ solve first order $\mathrm{A} \Delta \mathrm{Es}$ to which the theory developed in Sections II and III applies. In fact, in suitable parameter regimes $u(z)$ can be characterized as the unique minimal solution satisfying $u(0)=1$ and $|u(x)|=1$ for real $x$, whereas a reduced weight function $w_{r}(z)$ (closely related to $w(z)$ ) can be characterized in a similar way. It would 
take us too far afield to explain here how these A $\Delta$ Es (which are specified in Sections IV and V) emerge from the difference operators and their eigenfunctions. Instead, we refer to Ref. 1, p. 187, and Ref. 2, Subsection 4.3, for a derivation of the A $\Delta$ Es satisfied by $u(z)$ and $w(z)$, resp. (See also our forthcoming paper. ${ }^{13}$ )

From the viewpoint of special function theory, the $u$ - and $w$-functions are just simple combinations of the $G$-functions from Section III: Both functions are of the form $G(\cdots) G(\cdots) / G(\cdots) G(\cdots)$. The pertinent combinations, however, turn out to have quite remarkable properties, which reflect their origins in the context of analytic difference operators and eigenfunction transforms.

We study the functions $u(z)$ and $w(z)$ along similar lines, once more handling the hyperbolic, elliptic and trigonometric cases successively. In each case we first define the relevant function in terms of $G$-functions, read off some automorphy properties, and introduce some associated functions and/or parameter regimes. Then we study the functions in relation to the elementary A $\Delta E s$ they obey. As it happens, there is an additional elementary $\mathrm{A} \Delta \mathrm{E}$ pertaining to a parameter (essentially the coupling constant in the integrable system picture), which makes it possible to express $u(z)$ and $w(z)$ in terms of products of $s$-functions (i.e., $\operatorname{sh}(\cdot), s(\cdot)$ and $\sin (\cdot)$, resp.) for certain parameter values. In the hyperbolic and elliptic cases, these values are in fact dense in the parameter space.

After obtaining these elementary representations for special parameters, we return to the general case and derive various representations of a different character. At the end of each subsection we obtain a number of limits, whose existence is suggested by the formal limiting behavior of the difference Hamiltonians. Quite a few of these limits may be physically interpreted as nonrelativistic limits. For the scattering functions we also derive limits that may be viewed as classical limits. The zero step size results of Sections II and III are the main tools in controlling most of the limits-in particular the classical limits.

To conclude this introduction, we would like to point out that our results entail a great many nontrivial identities. As a rule, these identities are not spelled out: they follow from different representations for the same function. To be sure, quite a few of these formulas can be assembled via elementary identities-one may even assert that this is precisely what we have done in this paper. But this hindsight wisdom obscures what we view as the basic reason underlying most of the identities, namely, the uniqueness of minimal solutions to first order A $\Delta E s$ that admit such solutions.

To render the previous paragraph more concrete, we add an example. The sine-Gordon specialization of the $u$-function from Subsection IV A has been known in terms of the integral (4.30) for almost two decades (cf. Ref. 14 and references given there). Specifically, using our conventions, this $S$-matrix element reads

$$
u(\pi, \alpha, \pi / 2 ; z)=\exp \left(i \int_{0}^{\infty} \frac{d y}{y} \frac{\operatorname{sh}(\alpha-\pi / 2) y}{\operatorname{ch}(\pi y / 2) \operatorname{sh} \alpha y} \sin 2 y z\right), \quad|\operatorname{Im} 2 z|<d,
$$

with $d$ given by (4.32). (In point of fact, the integral occurred even earlier as a partition function of the six-vertex model, cf. Ref. 15.) Nevertheless, the result (4.28), expressing (1.19) as an elementary function for the dense set (4.27) of $\alpha$-values, is new. For $\alpha<\pi$ the resulting identity can be verified directly by noting that the rhs of (4.28) is a minimal solution to the $A \Delta E$ (4.6) with $\delta=-, a_{+}=\pi$ and $a_{-}=\alpha$, which moreover has value 1 and modulus 1 for $z=0$ and $z$ real, resp., just as (1.19). 


\section{GENERAL RESULTS ON ANALYTIC DIFFERENCE EQUATIONS}

\section{A. Preliminaries}

As announced in the Introduction, we are concerned with $\mathrm{A} \Delta \mathrm{Es}$ of the form

$$
\frac{F(z+i a / 2)}{F(z-i a / 2)}=\Phi(z), \quad a>0
$$

where $\Phi(z)$ is a function that is meromorphic in C (briefly: meromorphic). We shall call a function $F(z)$ a solution to (2.1) if and only if $F(z)$ is meromorphic in a strip $|\operatorname{Im} z|<s+a / 2, s$ $\in(0, \infty)$, and $F(z)$ satisfies $(2.1)$ for $|\operatorname{Im} z|<s$.

The first thing to note is that any solution thus defined extends to a meromorphic function. Indeed, one can extend $F(z)$ upwards strip by strip via

$$
F(z+i k a) \equiv \prod_{j=1}^{k} \Phi(z+(j-1 / 2) i a) \cdot F(z), \quad|\operatorname{Im} z| \leqslant a / 2,
$$

and downwards via

$$
F(z-i k a) \equiv \prod_{j=1}^{k} \frac{1}{\Phi(z-(j-1 / 2) i a)} \cdot F(z), \quad|\operatorname{Im} z| \leqslant a / 2 .
$$

Clearly, the quotient of two solutions to (2.1) is an $i a$-periodic meromorphic function.

Whenever $\Phi(x+i y), x, y \in \mathbb{R}$, converges to 1 for $y \rightarrow \infty$, uniformly for $x$ varying over arbitrary compact subsets of $\mathbb{R}$ and sufficiently fast, the infinite product

$$
F_{+}(z) \equiv \prod_{j=1}^{\infty} \frac{1}{\Phi(z+(j-1 / 2) i a)}
$$

defines a solution to (2.1). We shall refer to $F_{+}$as the upward iteration solution. It is readily seen that it is the only solution satisfying $F(x+i y) \rightarrow 1$ for $y \rightarrow \infty$. Similarly, the downward iteration solution

$$
F_{-}(z) \equiv \prod_{j=1}^{\infty} \Phi(z-(j-1 / 2) i a)
$$

exists provided $\Phi(x+i y) \rightarrow 1$ for $y \rightarrow-\infty$ (uniformly on $x$-compacts and sufficiently fast), and is the unique solution satisfying $F(x+i y) \rightarrow 1$ for $y \rightarrow-\infty$.

Consider, for example, the $\mathrm{A} \Delta \mathrm{Es}$ with right-hand sides

$$
\Phi_{1}(z)=\operatorname{ch} z, \quad \Phi_{2}(z)=1-\exp (i z-s), \quad \Phi_{3}(z)=1-\exp (i z+s), \quad s>0 .
$$

In the first case no iteration solution exists, whereas in the second and third cases $F_{+}$exists, but $F_{-}$does not.

Our main interest is in $\mathrm{A} \Delta \mathrm{Es}$ (or, equivalently, meromorphic functions $\Phi(z)$ ) that admit solutions with special properties in the strip $|\operatorname{Im} z| \leqslant a / 2$. Specifically, we shall restrict attention from now on to meromorphic functions $\Phi(z)$ that have no poles and zeros in a strip $|\operatorname{Im} z|<s$. Such functions and the associated $\mathrm{A} \Delta \mathrm{Es}(2.1)$ will be called regular. A solution to a regular $\mathrm{A} \Delta \mathrm{E}$ will be called regular iff it has no poles and zeros in $|\operatorname{Im} z| \leqslant a / 2$. In view of (2.2) and (2.3) it then actually has no poles and zeros in $|\operatorname{Im} z|<s+a / 2$. Clearly, the quotient of two regular solutions is 
an $i a$-periodic nowhere vanishing entire function. Note that the three $\mathrm{A} \Delta \mathrm{Es}$ defined by (2.6) are all regular; in the second case $F_{+}$is regular, whilst in the third case $F_{+}$is not (it has a pole in the set $i a / 2[-1,1])$.

It should be noticed that a regular solution is "maximally analytic," in the sense that it is free of poles and zeros in the strip $|\operatorname{Im} z| \leqslant a / 2$; its poles and zeros outside the latter strip are then determined by the $\mathrm{A} \Delta \mathrm{E}$ (2.1), and can be read off from (2.2) and (2.3), whenever the poles and zeros of $\Phi(z)$ are known. We shall be primarily concerned with a restricted type of $\mathrm{A} \Delta \mathrm{E}$, which admits regular solutions that are "minimal." To define this notion, we observe that a regular solution $F(z)$ to (2.1) admits a one-valued analytic logarithm in $|\operatorname{Im} z|<s+a / 2$. We call $F$ a minimal solution iff $\ln F(z)$ is polynomially bounded in $|\operatorname{Im} z| \leqslant a / 2$. That is, there exist $c, d>0$ and $k \in \mathbb{N}$ such that

$$
|\ln F(z)|<c+d|z|^{k}, \quad \forall z \in\{|\operatorname{Im} z| \leqslant a / 2\}
$$

Taking $z=x \in \mathbb{R}$ in (2.1), we deduce

$$
|\Phi(x)|^{\delta}<\exp \left(2 c+2 d|x|^{k}\right), \quad \forall x \in \mathbb{R}, \quad \delta= \pm 1 .
$$

Thus, $\Phi(z)$ must satisfy (2.8) for minimal solutions to exist.

To show that $\mathrm{A} \Delta \mathrm{Es}$ admitting minimal solutions are by no means exceptional, let $g(z)$ be any meromorphic function that is analytic in $|\operatorname{Im} z|<s+a / 2$ and polynomially bounded in $|\operatorname{Im} z| \leqslant a / 2$. Then the $\mathrm{A} \Delta \mathrm{E}$ with rhs $\Phi(z) \equiv \exp (g(z+i a / 2)-g(z-i a / 2))$ admits a minimal solution, viz., $F(z)=\exp (g(z))$. It is also to be noted that the right-hand side functions $\Phi(z)$ of $(2.1)$ that admit minimal solutions form a group: If $F(z)$ is a minimal solution to $(2.1)$, then $1 / F(z)$ is a minimal solution to (2.1) with $\Phi \rightarrow 1 / \Phi$, and if $F_{1}, F_{2}$ are minimal solutions to A $\Delta$ Es (2.1) with rhs $\Phi_{1}, \Phi_{2}$, resp., then $F(z)=F_{1}(z) F_{2}(z)$ is a minimal solution to $(2.1)$ with $\Phi(z)=\Phi_{1}(z) \Phi_{2}(z)$.

A minimal solution is not only maximally analytic (since it is regular by definition), but also has the slowest increase to $\infty$ and/or decrease to 0 for $\operatorname{Re} z \rightarrow \pm \infty$ in the strip $|\operatorname{Im} z| \leqslant a / 2$ that is compatible with (2.1). This will be clear from the following theorem, which shows, moreover, that minimal solutions have "minimal ambiguity."

Theorem II.1: Assume that the meromorphic function $\Phi(z)$ is regular and satisfies (2.8). Let $F_{1}(z)$ and $F_{2}(z)$ be minimal solutions to the $A \Delta E(2.1)$. Then there exist $C \in \mathbb{C}^{*}$ and $l \in \mathbb{Z}$ such that

$$
F_{1}(z) / F_{2}(z)=C \exp (2 \pi l z / a)
$$

If $F_{1}(z)$ and $F_{2}(z)$ are bounded away from 0 and $\infty$ on $\mathbb{R}$, then one has $l=0$ in (2.9). If $\Phi(z)$ is even, then for all minimal solutions $F(z)$ the function $F(z) F(-z)$ is constant. If $\Phi(0)=1$ and the function $\Phi(z) \Phi(-z)$ equals 1 , then for any minimal solution $F(z)$ there exists $k \in \mathbb{Z}$ such that $\exp (2 \pi k z / a) F(z)$ is an even minimal solution.

Proof: Since $F_{1}$ and $F_{2}$ are minimal, they are a fortiori regular. Therefore, $F_{1}(z) / F_{2}(z)$ is an $i a$-periodic entire function $q(z)$ without zeros. Hence there exists $l \in \mathbb{Z}$ such that the function $q_{0}(z) \equiv q(z) \exp (-2 \pi l z / a)$ has zero winding number around 0 as $z$ goes from $z_{0}$ to $z_{0}+i a$.

To prove that $q_{0}(z)$ is constant, we note that it can be written $\exp [r(z)]$, with $r(z)$ an $i a$-periodic entire function. Since $F_{1}$ and $F_{2}$ are minimal, $r(z)$ is polynomially bounded:

$$
|r(z)| \leqslant C_{1}+C_{2}|z|^{k}, \quad|\operatorname{Im} z| \leqslant a / 2
$$

It is not hard to see that this entails constancy of $r(z)$. (Indeed, we can, for instance, argue as follows. Since $r(z)$ is $i a$-periodic and entire, it can be written $\Sigma_{n \in \mathbb{Z}} c_{n} w^{n} \equiv s(w)$, where 
$w \equiv \exp (2 \pi z / a)$, and where the series converges for $w \in \mathbb{C}^{*}$. In view of the bound (2.10), the function $w s(w)$ has limit 0 for $w \rightarrow 0$, so it is analytic at $w=0$. Hence, $c_{n}=0$ for $n<0$. Similarly, since (2.10) entails $s(w) / w \rightarrow 0$ for $w \rightarrow \infty$, we deduce $c_{n}=0$ for $n>0$.)

We have now proved the first assertion (2.9). The second one is then clear from (2.9). Now assume $\Phi(z)$ is even and $F(z)$ is a minimal solution. Consider the function $G(z) \equiv 1 / F(-z)$. It satisfies

$$
\frac{G(z+i a / 2)}{G(z-i a / 2)}=\frac{F(-z+i a / 2)}{F(-z-i a / 2)}=\Phi(-z)=\Phi(z),
$$

so it is a solution, too. From minimality of $F$ one easily deduces minimality of $G$, so (2.9) entails there exists $l \in \mathbb{Z}$ such that $F(z) / G(z)=C \exp (2 \pi l z / a)$. But the function on the lhs equals $F(z) F(-z)$ and hence is even. Therefore, we have $l=0$ and the third assertion follows.

To prove the last assertion, consider the function $H(z) \equiv F(-z)$. It satisfies

$$
\frac{H(z+i a / 2)}{H(z-i a / 2)}=\frac{F(-z-i a / 2)}{F(-z+i a / 2)}=1 / \Phi(-z)=\Phi(z),
$$

and so it is a second minimal solution. Thus we must have $F(-z)=C \exp (2 \pi l z / a) F(z)$. Putting $z=0$ yields $C=1$ and putting $z=i a / 2$ yields $(-)^{l} \Phi(0)=1$, so that $l$ is even. But then $\exp (2 \pi k z / a) F(z)$ with $k \equiv l / 2$ is an even minimal solution.

Thus far, we have been dealing with meromorphic A $\Delta E$ Es of the multiplicative form (2.1). To study these in more detail and, in particular, to construct minimal solutions, it turns out to be convenient to also consider $\mathrm{A} \Delta \mathrm{Es}$ of the additive form

$$
f(z+i a / 2)-f(z-i a / 2)=\phi(z), \quad a>0 .
$$

Here, $\phi(z)$ is assumed to be meromorphic in a strip $|\operatorname{Im} z|<s, s \in(0, \infty)$, and we restrict attention to functions $f(z)$ that are meromorphic in the strip $|\operatorname{Im} z|<s+a / 2$ and that satisfy (2.13) for $|\operatorname{Im} z|<s$; the term "solution to (2.13)" will be used only for such functions. The function $\phi(z)$ and the associated $\mathrm{A} \Delta \mathrm{E}$ (2.13) will be termed regular iff $\phi(z)$ is analytic in $|\operatorname{Im} z|<s$, and a solution $f(z)$ to a regular $\mathrm{A} \Delta \mathrm{E}$ will be called regular iff $f(z)$ is analytic in $|\operatorname{Im} z|<s+a / 2$.

Obviously, taking logarithms of a regular $\mathrm{A} \Delta \mathrm{E}$ of the multiplicative form (2.1) leads to a regular $\mathrm{A} \Delta \mathrm{E}$ of the additive form (2.13). Since the meromorphic function $\Phi(z)$ may have zeros and/or poles for $|\operatorname{Im} z| \geqslant s$, its logarithm may have branch points for $|\operatorname{Im} z| \geqslant s$. Such branch points are irrelevant for studying the $\mathrm{A} \Delta \mathrm{E}(2.1)$, and therefore we restrict attention to the strip $|\operatorname{Im} z|<s$ in the additive case.

The above-mentioned notions and results connected to (2.1) have obvious analogs for (2.13). In particular, a regular solution $f(z)$ to a regular $\mathrm{A} \Delta \mathrm{E}$ (2.13) will be termed minimal iff it is polynomially bounded in $|\operatorname{Im} z| \leqslant a / 2$, and a necessary condition for the existence of minimal solutions is that $\phi(z)$ be polynomially bounded on $\mathbb{R}$. Of course, in the additive case two minimal solutions can only differ by a constant, cf. the proof of Theorem II.1.

Let us now compare the above to the older literature on first order $\mathrm{A} \Delta \mathrm{Es}$, cf. in particular Refs. 5-8. Here, one usually considers additive $\mathrm{A} \Delta \mathrm{Es}$ of the form

$$
u(w+1)-u(w)=b(w) .
$$

Of course, these are essentially equal to (2.13), as follows by making the change of variables $z=i a(w+1 / 2)$ in $(2.13)$. But these different conventions reflect a different emphasis. Indeed, our main interest is in the behavior of $\phi(z)$ and associated solutions in the strip $|\operatorname{Im} z| \leqslant a / 2$; in particular, we shall obtain representations for minimal solutions that hold true in this strip, cf. the next two subsections. 
By contrast, Nörlund ${ }^{5}$ singles out the "principal solution" (Hauptlösung) to (2.14) by imposing conditions on $b(w)$ for $\operatorname{Re} w \rightarrow \infty$; accordingly, his principal solution can be characterized among all other solutions by its having the slowest possible increase for $\operatorname{Re} w \rightarrow \infty$. The principal solution equals the obvious iteration solution to (2.14) whenever $b(w)$ goes to 0 sufficiently fast for $\operatorname{Re} w \rightarrow \infty$, but it can be defined for larger classes of right-hand sides by modifying the iteration, cf. loc. cit. Chapters 3 and 4. As we have already seen [cf. $\Phi_{3}(z)$ in (2.6)], an iteration solution need not be regular, and so, a fortiori, it need not be minimal. Moreover, minimality concerns the asymptotics for $\operatorname{Im} w \rightarrow \pm \infty$, and not $\operatorname{Re} w \rightarrow \pm \infty$.

If one writes the hyperbolic and elliptic $\mathrm{A} \Delta \mathrm{Es}$ occurring below (for which we construct minimal solutions) in the form (2.14), then Nörlund's conditions are violated, and no principal solution exists. On the other hand, Nörlund's conditions allow right-hand side functions $\phi(z)$ in (2.13) that are not polynomially bounded on $\mathbb{R}$; in that case, (2.13) does not admit minimal solutions. For the regular trigonometric and rational A $\Delta$ Es occurring below, both Nörlund's and our solution methods apply, and the principal solution is then a minimal solution. Our Fourier series representation for the trigonometric case is however very different from the representations for the principal solution occurring in Ref. 5 .

\section{B. Fourier transform solutions}

In this subsection we obtain minimal solutions to a large class of $\mathrm{A} \Delta \mathrm{Es}$ by exploiting Fourier transformation on $L^{2}(\mathbb{R})$. (This class contains the $\mathrm{A} \Delta \mathrm{Es}$ that occur in the hyperbolic context, cf. the Introduction.) Our normalization reads

$$
\hat{\Psi}(y) \equiv \frac{1}{2 \pi} \int_{-\infty}^{\infty} d x \Psi(x) e^{i x y}
$$

so that

$$
\Psi(x)=\int_{-\infty}^{\infty} d y \hat{\Psi}(y) e^{-i x y}
$$

Of course, we may and will use the definition (2.15) for $\Psi \in L^{1}(\mathbb{R})$, too; in this case, recall $\hat{\Psi}(y) \rightarrow 0$ for $y \rightarrow \pm \infty$ (Riemann-Lebesgue lemma). We also have occasion to use the distributional Fourier transform

$$
\int_{-\infty}^{\infty} d y e^{-2 i y z} \mathrm{P} \frac{1}{\operatorname{sh} a y}=-\frac{i \pi}{a} \operatorname{th}\left(\frac{\pi z}{a}\right), \quad|\operatorname{Im} z|<a / 2,
$$

where $\mathrm{P}$ denotes the principal value. (This formula can be verified by a straightforward contour integration.)

Theorem II.2: Assume $\phi(z)$ is a function with the following properties:

$$
\begin{gathered}
\phi(z) \text { is analytic in a strip }|\operatorname{Im} z|<s, s \in(0, \infty), \\
\phi(x) \in L^{1}(\mathbb{R}), \\
\hat{\phi}(y) \in L^{1}(\mathbb{R}), \\
\hat{\phi}(y)=O(y), y \rightarrow 0 .
\end{gathered}
$$

Then the $A \Delta E$

$$
f(z+i a / 2)-f(z-i a / 2)=\phi(z), \quad a>0, \quad|\operatorname{Im} z|<s,
$$


has a unique solution $f(a ; z)$ such that

$$
\begin{gathered}
f(a ; z) \text { is analytic in the strip }|\operatorname{Im} z|<s+a / 2, \\
f(a ; z) \text { is bounded in the strip }|\operatorname{Im} z| \leqslant a / 2, \\
\lim _{x \rightarrow \pm \infty} f(a ; x+i t)=0, \quad t \in[-a / 2, a / 2] .
\end{gathered}
$$

Explicitly, this solution can be written as

$$
f(a ; z)=\int_{-\infty}^{\infty} d y \frac{\hat{\phi}(2 y)}{\operatorname{sh} a y} e^{-2 i y z}, \quad|\operatorname{Im} z| \leqslant a / 2,
$$

or as

$$
f(a ; z)=\frac{1}{2 i a} \int_{-\infty}^{\infty} d u \phi(u) \operatorname{th} \frac{\pi}{a}(z-u), \quad|\operatorname{Im} z|<a / 2 .
$$

It satisfies the addition formula

$$
f\left(\frac{a}{k} ; z\right)=\sum_{j=1}^{k} f\left(a ; z+\frac{i a}{2 k}(k+1-2 j)\right) .
$$

If $\phi(z)$ is even/odd, then $f(a ; z)$ is oddleven. Finally, let $\psi(x)$ be the following primitive of $\phi(x), x \in \mathbb{R}$ :

$$
\psi(x)=\frac{1}{2}\left(\int_{-\infty}^{x} d u \phi(u)-\int_{x}^{\infty} d u \phi(u)\right)
$$

Then one has

$$
\operatorname{limiaf}_{a \rightarrow 0}(a ; z)=\psi(z)
$$

uniformly on compact subsets of the strip $|\operatorname{Imz}|<s$.

Proof: First we prove uniqueness. Thus, let $d(z)$ be the difference of two solutions to $(2.22)$ with properties (2.23)-(2.25). Then $d(z)$ is an analytic function in $|\operatorname{Im} z|<s+a / 2$, satisfying $d(z+i a / 2)=d(z-i a / 2)$ for $|\operatorname{Im} z|<s$. Therefore, $d(z)$ extends to an $i a$-periodic entire function. By virtue of $(2.24), d(z)$ is bounded in the period strip $|\operatorname{Im} z| \leqslant a / 2$, so $d(z)$ is constant in view of Liouville's theorem. On account of (2.25) this constant equals 0 , so uniqueness follows.

Next, we use (2.19) and (2.21) to infer that the function $\hat{\phi}(2 y)$ /shay is bounded and satisfies

$$
|\hat{\phi}(2 y) / \operatorname{sh} a y|=o\left(e^{-a|y|}\right), \quad y \rightarrow \pm \infty .
$$

Thus, defining a function $f(z)$ by the rhs of (2.26), it is clear that $f(z)$ is analytic in $|\operatorname{Im} z|<a / 2$ and that $f(x+i t)$ converges to 0 for $x \rightarrow \pm \infty$ and $|t|<a / 2$. Moreover, using also (2.20), we infer that the functions

$$
b_{ \pm}(x) \equiv \int_{-\infty}^{\infty} d y \frac{\hat{\phi}(2 y)}{\text { shay }} e^{ \pm a y} e^{-2 i y x}, \quad x \in \mathbb{R},
$$

are continuous and vanish at $\pm \infty$, and that we have 


$$
\lim _{t \uparrow 1} f(x \pm i t a / 2)=b_{ \pm}(x)
$$

uniformly on $\mathbb{R}$.

Now consider the auxiliary function

$$
A(z) \equiv f(z-i a / 2)+\phi(z)
$$

Clearly, $A(z)$ is analytic in the strip

$$
S_{+} \equiv\{z \in \mathbb{C} \mid \operatorname{Im} z \in(0, \gamma)\}, \quad \gamma \equiv \min (s, a),
$$

and $A(x+i \epsilon)$ converges to $b_{-}(x)+\phi(x)$ as $\epsilon \downarrow 0$, uniformly for $x$ in compact subsets of $\mathbb{R}$. But from (2.32) we have

$$
b_{+}(x)-b_{-}(x)=2 \int_{-\infty}^{\infty} d y \hat{\phi}(2 y) e^{-2 i y x}=\phi(x),
$$

so this boundary value is equal to $b_{+}(x)$. On the other hand, the function $f(z+i a / 2)$ is analytic in the strip $\operatorname{Im} z \in(-a, 0)$ and converges uniformly on $\mathbb{R}$ to $b_{+}(x)$ as $\operatorname{Im} z \uparrow 0$. Consequently, we may invoke Painlevé's lemma to deduce that $f(z+i a / 2)$ extends to an analytic function in $\operatorname{Im} z \in(-a, \gamma)$, which coincides with $A(z)$ when $z \in S_{+}$. That is, the $\mathrm{A} \Delta \mathrm{E}(2.22)$ holds true for $z \in S_{+}$.

We may now exploit (2.22) for $z \in S_{+}$to deduce that $f(z)$ extends to an analytic function in $|\operatorname{Im} z|<s+a / 2$. Since the functions $f(x \pm i a / 2)$ equal $b_{ \pm}(x)$, they converge to 0 for $x \rightarrow \pm \infty$. Moreover, recalling the definition of $f(z)$, we obtain

$$
|f(z)| \leqslant \int_{-\infty}^{\infty} d y \frac{|\hat{\phi}(2 y)|}{|\operatorname{sh} a y|} e^{a|y|}, \quad|\operatorname{Im} z| \leqslant a / 2,
$$

and in view of (2.20) and (2.21) the rhs is finite. Therefore, the rhs of (2.26) defines a solution to (2.22) with the properties (2.23)-(2.25).

Next, we prove (2.27). Replacing the integral in (2.26) by a principal value integral, and the functions $\phi$ and $\hat{\phi}$ in (2.27) and (2.26) by a Schwartz space function $\chi$ and its Fourier transform $\hat{\chi}$, resp., the equality of the resulting integrals is clear from (2.17) and the Plancherel relations. Since $S(\mathbb{R})$ is dense in $L^{1}(\mathbb{R})$, we deduce (2.27) from (2.26).

The function at the rhs of (2.28) obviously solves (2.22) with $a$ replaced by $a / k$. Since it also has the properties $(2.23)-(2.25)$ that uniquely determine $f(a / k ; z)$, we obtain (2.28). Alternatively, (2.28) follows directly from the representation (2.26) by using the elementary identity

$$
\sum_{j=1}^{k} \exp \left(\frac{a y}{k}(k+1-2 j)\right)=\frac{\operatorname{sh}(a y)}{\operatorname{sh}(a y / k)} .
$$

The parity assertion can be read off from both of the representations (2.26) and (2.27).

It remains to prove the last assertion. To this end we first observe that the representation (2.27) entails that (2.30) holds true pointwise for $z=x \in \mathbb{R}$. Next, we use the bound (2.37) and the assumptions (2.20) and (2.21) to infer that the function $a f(a ; z)$ remains bounded by an $a$-independent constant in the strip $|\operatorname{Im} z| \leqslant a / 2$ as $a \rightarrow 0$. By iteration of the $\mathrm{A} \Delta \mathrm{E}(2.22)$ we now deduce that $a f(a ; z)$ remains bounded in compact subsets of the strip $|\operatorname{Im} z|<s$ as $a \rightarrow 0$. Therefore, the last assertion follows from Vitali's theorem. 
For our purposes the conditions (2.18)-(2.21) on $\phi(z)$ are sufficiently weak. In general, however, the conditions (2.20) and (2.21) may be difficult to check. Requiring solely (2.18) and (2.19), the rhs of (2.27) defines a function $f(z)$ that is clearly analytic in the strip $|\operatorname{Imz}|<a / 2$ and that satisfies

$$
\lim _{x \rightarrow \pm \infty} f(x \pm i t)= \pm \frac{1}{2 i a} \int_{-\infty}^{\infty} d u \phi(u), \quad t \in(-a / 2, a / 2) .
$$

We conjecture that this function is in fact a solution to (2.22) satisfying (2.23) and (2.24).

Returning to the assumptions of the theorem, let us note that (2.21) entails that the primitive $\psi(x)$ (2.29) vanishes at $\pm \infty$. Thus, writing $\phi(u)=\psi^{\prime}(u)$ in the representation (2.27), and integrating by parts, we obtain the formula

$$
f(a ; z)=\frac{\pi}{2 i a^{2}} \int_{-\infty}^{\infty} d u \frac{\psi(u)}{\operatorname{ch}^{2} \frac{\pi}{a}(z-u)}, \quad|\operatorname{Im} z|<a / 2 .
$$

Comparing this representation to Eq. (14) in Chapter 4 of Nörlund's monograph, ${ }^{5}$ one sees that the solution $f(a ; z)$ and Nörlund's principal solution differ only by a constant whenever $\phi(z)$ satisfies not only the assumptions of Theorem II.2, but also the various restrictions that Nörlund needs for his principal solution to exist and admit the representation (14) in loc. cit. (As already mentioned, his assumptions on $\phi(z)$ are quite different from ours, cf. the discussion after (2.14).)

It is also of interest to observe that the assumptions (2.19)-(2.21) entail that $\hat{\phi}(y)$ is an $L^{2}(\mathbb{R})$-function in the domain of the unbounded self-adjoint multiplication operator $1 / \operatorname{sh}(a y / 2)$. From this point of view the function $f(a ; x), x \in \mathbb{R}$, given by (2.26), is the obvious $L^{2}(\mathbb{R})$-solution to (2.22) with $z \in \mathbb{R}$, reinterpreted as a Hilbert space equation. (Indeed, the function $\hat{f}(a ; y)$-being equal to $\hat{\phi}(y) / 2 \operatorname{sh}(a y / 2)$-is in the domain of multiplication by $\exp ( \pm a y / 2)$.)

We proceed by generalizing the above key result Theorem II.2. We shall detail this generalization in the multiplicative context (2.1); the additive version will be clear from this.

Theorem II.3: Assume $\Phi(z)$ is a meromorphic function that has no poles and zeros in the strip $|\operatorname{Im} z|<s$ for some $s \in(0, \infty)$. Setting

$$
\phi_{l}(z) \equiv\left(\frac{d}{d z}\right)^{l} \ln \Phi(z), \quad l \in \mathbb{N}
$$

assume there exists $k \in \mathbb{N}^{*}$ such that $\phi(z) \equiv \phi_{k}(z)$ satisfies (2.18)-(2.21). Then the $A \Delta E$

$$
\frac{F(z+i a / 2)}{F(z-i a / 2)}=\Phi(z)
$$

admits minimal solutions. Any minimal solution can be written as

$$
F(z)=\exp (e(z)+P(z))
$$

where

$$
e(z) \equiv \int_{-\infty}^{\infty} d y \frac{\hat{\phi}(2 y)}{\operatorname{sh} a y}(-2 i y)^{-k}\left(e^{-2 i y z}-\sum_{j=0}^{k-1} \frac{(-2 i y z)^{j}}{j !}\right), \quad|\operatorname{Im} z| \leqslant a / 2,
$$

and 


$$
P(z) \equiv \sum_{j=0}^{k} c_{j} z^{j} / j !, \quad c_{0}, \ldots, c_{k} \in \mathbb{C} .
$$

The coefficients $c_{2}, \ldots, c_{k}$ are uniquely determined, whereas $c_{1}$ is uniquely determined $\bmod 2 \pi / a$.

Proof: Consider the A $\Delta \mathrm{Es}$

$$
f_{l}(z+i a / 2)-f_{l}(z-i a / 2)=\phi_{l}(z), \quad l=0, \ldots, k .
$$

By virtue of Theorem II.2 the function

$$
f_{k}(z) \equiv \int_{-\infty}^{\infty} d y \frac{\hat{\phi}(2 y)}{\operatorname{sh} a y} e^{-2 i y z}, \quad|\operatorname{Im} z| \leqslant a / 2,
$$

admits an analytic continuation to $|\operatorname{Im} z|<s+a / 2$ and satisfies (2.46) with $l=k$. Introducing

$$
f_{k-1}(z) \equiv c_{k} z+\int_{0}^{z} d s f_{k}(s), \quad c_{k} \in \mathbb{C},
$$

we infer that the rhs of the resulting equation

$$
f_{k-1}(z+i a / 2)-f_{k-1}(z-i a / 2)=i a c_{k}+\int_{z-i a / 2}^{z+i a / 2} d s f_{k}(s)
$$

equals $\phi_{k-1}(z)$ for a suitable choice of $c_{k}$ [since its $z$-derivative equals $\phi_{k}(z)$ ]; specifically, we may and will choose $c_{k}$ such that

$$
i a c_{k}+\int_{-i a / 2}^{i a / 2} d s f_{k}(s)=\phi_{k-1}(0)
$$

Proceeding recursively, we obtain functions $f_{k}(z), f_{k-1}(z), \ldots, f_{0}(z)$ related by

$$
f_{l-1}(z)=c_{l} z+\int_{0}^{z} d s f_{l}(s), \quad l=1, \ldots, k,
$$

with $c_{l}$ given by

$$
c_{l}=\frac{1}{i a}\left(\phi_{l-1}(0)-\int_{-i a / 2}^{i a / 2} d s f_{l}(s)\right), \quad l=1, \ldots, k .
$$

Then $f_{l}(z), l \in\{0, \ldots, k\}$, is analytic in $|\operatorname{Im} z|<s+a / 2$ and is a minimal solution to (2.46). Moreover, from (2.51) and (2.47) one easily sees that $f_{0}(z)$ equals the sum of $e(z)$ and a polynomial of degree $\leqslant k$. The proof can now be completed by invoking Theorem II.1.

In Appendix A we show (among other things) how the above results can be used to arrive at the psi and gamma functions, and derive various salient features along the way. Here, we add two applications exemplifying the above, yielding identities we have occasion to use later on. First, consider the function

$$
F(z)=\operatorname{cth} z-\frac{\pi}{a} \operatorname{cth} \frac{\pi z}{a}
$$

It satisfies the $\mathrm{A} \Delta \mathrm{E}$ 


$$
F(z+i a / 2)-F(z-i a / 2)=\operatorname{cth}(z+i a / 2)-\operatorname{cth}(z-i a / 2) \equiv \chi(z) .
$$

Inverting (2.17) yields the distributional Fourier transforms

$$
\int_{-\infty}^{\infty} d x \operatorname{cth} \alpha(x \pm i \beta) e^{i x y}=\frac{i \pi}{\alpha} \mathrm{p} \frac{\exp \pm(-\pi y / 2 \alpha+\beta y)}{\operatorname{sh}(\pi y / 2 \alpha)}, \quad \alpha>0, \quad \beta \in(0, \pi / \alpha),
$$

so we have

$$
\hat{\chi}(y)=\frac{1}{2 \pi} \int_{-\infty}^{\infty} d x \chi(x) e^{i x y}=i \frac{\operatorname{sh} y(a-\pi) / 2}{\operatorname{sh} y \pi / 2}, \quad a \in(0,2 \pi) .
$$

Thus, $\chi(z)$ satisfies the assumptions (2.18)-(2.20), but not (2.21). But $\phi(z) \equiv \chi^{\prime}(z)$ does satisfy (2.18)-(2.21), since

$$
\hat{\phi}(y)=y \frac{\operatorname{sh} y(a-\pi) / 2}{\operatorname{sh} y \pi / 2}
$$

Therefore, we obtain a solution

$$
f(z)=4 \int_{0}^{\infty} d y \frac{y \operatorname{sh}(a-\pi) y}{\operatorname{sh} a y \operatorname{sh} \pi y} \cos 2 y z
$$

to the $\mathrm{A} \Delta \mathrm{E}$ (2.22). Now since $F^{\prime}(z)$ satisfies (2.22), too, and obviously has the properties (2.23)(2.25), we must have $f(z)=F^{\prime}(z)$, by uniqueness. Integrating the resulting identity w.r.t. $z$, we obtain

$$
\operatorname{cth} z-\frac{\pi}{a} \operatorname{cth} \frac{\pi z}{a}=2 \int_{0}^{\infty} d y \frac{\operatorname{sh}(a-\pi) y}{\operatorname{sh} a y \operatorname{sh} \pi y} \sin 2 y z
$$

Here we have $a \in(0,2 \pi)$ in view of the restriction in (2.56). But for $z \in \mathbb{R}$ the integral converges for any $a>0$, and so it readily follows that (2.59) holds for any $a>0$ (taking $|\operatorname{Im} z|$ small enough, of course). Integrating once more now yields

$$
\ln (\operatorname{sh} z)-\ln \left(\frac{a}{\pi} \operatorname{sh} \frac{\pi z}{a}\right)=\int_{0}^{\infty} \frac{d y}{y} \frac{\operatorname{sh}(a-\pi) y}{\operatorname{sh} a y \operatorname{sh} \pi y}(1-\cos 2 y z), \quad a>0 .
$$

Second, consider the function

$$
h(z) \equiv \frac{z}{a} \operatorname{cth} \frac{\pi z}{a} .
$$

It satisfies the $\mathrm{A} \Delta \mathrm{E}$

$$
h(z+i a / 2)-h(z-i a / 2)=i \operatorname{th} \frac{\pi z}{a} .
$$

Therefore, $h^{\prime \prime}(z)$ satisfies the $\mathrm{A} \Delta \mathrm{E}$

$$
f(z+i a / 2)-f(z-i a / 2)=\frac{i \pi}{a} \frac{d}{d z}\left(1 / \operatorname{ch}^{2} \frac{\pi z}{a}\right) \equiv \phi(z) .
$$


Now one readily verifies

$$
\hat{\phi}(y)=\frac{a y^{2}}{2 \pi \operatorname{sh}(a y / 2)}, \quad a>0
$$

so $\phi(z)$ satisfies the assumptions of Theorem II.2. The resulting solution

$$
f(z)=\frac{4 a}{\pi} \int_{0}^{\infty} d y \frac{y^{2}}{\operatorname{sh}^{2} a y} \cos 2 y z
$$

must then be equal to $h^{\prime \prime}(z)$, since $h^{\prime \prime}(z)$ clearly has the properties (2.23)-(2.25). Integrating twice w.r.t. $z$ we now obtain

$$
\pi z \operatorname{cth} \frac{\pi z}{a}=a+a^{2} \int_{0}^{\infty} d y \frac{(1-\cos 2 y z)}{\operatorname{sh}^{2} a y}, \quad a>0 .
$$

The identities (2.66) and (2.60) can be combined to evaluate integrals occurring below. First, they entail that for $a \in(0, \pi)$ one has

$$
\frac{a \pi}{a-\pi} \ln \left(\frac{\pi \operatorname{sh} z}{a \operatorname{sh} \frac{\pi z}{a}}\right)-\pi z \operatorname{cth} \frac{\pi z}{a}+a=\int_{0}^{\infty} d y\left(\frac{a \pi \operatorname{sh}(a-\pi) y}{(a-\pi) y \operatorname{sh} a y \operatorname{sh} \pi y}-\frac{a^{2}}{\operatorname{sh}^{2} a y}\right)(1-\cos 2 y z) .
$$

Taking $z \rightarrow \infty$ and using the Riemann-Lebesgue lemma we obtain the integral

$$
\frac{a \pi}{a-\pi} \ln \frac{\pi}{a}+a=\int_{0}^{\infty} d y\left(\frac{a \pi \operatorname{sh}(a-\pi) y}{(a-\pi) y \operatorname{sh} a y \operatorname{sh} \pi y}-\frac{a^{2}}{\operatorname{sh}^{2} a y}\right) .
$$

Adding the elementary integral

$$
\int_{0}^{\infty} d y\left(\frac{a^{2}}{\operatorname{sh}^{2} a y}-\frac{1}{y^{2}}\right)=-a
$$

yields

$$
\ln \frac{\pi}{a}=\int_{0}^{\infty} \frac{d y}{y}\left(\frac{\operatorname{sh}(a-\pi) y}{\operatorname{sh} a y \operatorname{sh} \pi y}-\frac{(a-\pi)}{a \pi y}\right),
$$

and combining this with $(2.60)$ we get

$$
\ln \left(\operatorname{sh} \frac{\pi z}{a}\right)-\ln (\operatorname{sh} z)=\int_{0}^{\infty} \frac{d y}{y}\left(\frac{\operatorname{sh}(a-\pi) y}{\operatorname{sh} a y \operatorname{sh} \pi y} \cos 2 y z-\frac{(a-\pi)}{a \pi y}\right), \quad a>0 .
$$

Just as in the above examples, $\mathrm{A} \Delta \mathrm{Es}$ with $a$-dependent right-hand side functions will be encountered later on. The last theorem of this subsection concerns the limit $a \rightarrow 0$ in this setting. It is convenient to use the assumptions of Theorem II.2 as a starting point; corresponding results in the slightly more general context of Theorem II. 3 can then be obtained by $k$-fold integration.

Specifically, we consider an $\mathrm{A} \Delta \mathrm{E}$ of the form

$$
f(z+i a / 2)-f(z-i a / 2)=\phi_{a}(z), \quad a>0,
$$


where $\phi_{a}(z)$ satisfies the assumptions (2.18)-(2.21) for any $a \in\left(0, a_{0}\right]$. (Of course, the choice of $a_{0}$ is irrelevant for the limit $a \rightarrow 0$.) We allow dependence of the maximal number $s_{m} \in(0, \infty]$ in (2.18) on $a$; in particular, one may have $s_{m} \rightarrow 0$ as $a \rightarrow 0$. However, we do assume that for any $a \in\left(0, a_{0}\right]$ the function $\phi_{a}(z)$ is analytic in the open right half plane

$$
\mathscr{R}_{0} \equiv\{z \in \mathbb{C} \mid \operatorname{Re} z>0\}
$$

Moreover, we assume that for any compact $K \subset \mathscr{B}_{0}$ there exists $C_{K}>0$ with

$$
\left|\phi_{a}(z)-a \chi(z)\right| \leqslant C_{K} a^{2}, \quad \forall(a, z) \in\left(0, a_{0}\right] \times K,
$$

where $\chi(z)$ is analytic in $\mathscr{R}_{0}$.

Now let $f_{a}(z)$ be the unique solution to (2.72) given by Theorem II.2 (with $\phi(z) \rightarrow \phi_{a}(z)$, of course). Thus, $f_{a}(z)$ is analytic in the strip $|\operatorname{Im} z|<a / 2+s_{m}(a)$ and in $\mathscr{R}_{0}$. We are now in the position to state the next result.

Theorem II.4: In addition to the above assumptions, let

$$
\left|f_{a}(z)\right| \leqslant C_{\delta, M}, \quad \forall(a, z) \in\left(0, a_{0}\right] \times\{z \in \mathbb{C}|\operatorname{Re} z \in[\delta, M],| \operatorname{Im} z \mid \leqslant a / 2\},
$$

for any $\delta>0$ and $M>\delta$, and let the pointwise limit

$$
\lim _{a \downarrow 0} f_{a}(z) \equiv f(z)
$$

exist for any $z \in(0, \infty)$. Then $f_{a}(z)$ converges uniformly on compact subsets of $\mathscr{R}_{0}$ to a function $f(z)$ that is analytic in $\mathscr{R}_{0}$. Moreover, one has

$$
f^{\prime}(z)=-i \chi(z), z \in \mathscr{R}_{0}
$$

with $\chi(z)$ defined by (2.74).

Proof: Upward iteration of the $\mathrm{A} \Delta \mathrm{E}$ (2.72) yields

$$
f_{a}(z+i L a)=f_{a}(z)+\sum_{j=1}^{L} \phi_{a}(z+(j-1 / 2) i a), \quad|\operatorname{Im} z| \leqslant a / 2 .
$$

Choosing

$$
L=N\left[a^{-1}\right], \quad \operatorname{Re} z \in[\delta, M], \quad 0<\delta<M,
$$

in this equation, the arguments of $\phi_{a}$ occurring on the rhs stay in a closed rectangle $K(N, \delta, M) \subset \mathscr{R}_{0}$ as $a \downarrow 0$. Thus we may invoke the bounds (2.74) and (2.75) to conclude that $f_{a}(z)$ remains bounded for $\operatorname{Re} z \in[\delta, M], \operatorname{Im} z \in[0, N]$, as $a \downarrow 0$. Similarly, iterating downwards $L$ times and requiring (2.79), we deduce that $f_{a}(z)$ remains bounded for $\operatorname{Re} z \in[\delta, M], \operatorname{Im} z$ $\in[-N, 0]$.

Combining uniform boundedness of $f_{a}(z)$ on compacts of $\mathscr{B}_{0}$ with the pointwise convergence assumption (2.76), it follows from Vitali's theorem that $f_{a}(z)$ converges uniformly on compacts of $\mathscr{B}_{0}$ to a function $f(z)$ that is analytic in $\mathscr{B}_{0}$. Therefore, it remains to prove (2.77).

To this end, we use (2.72) to write

$$
\frac{\phi_{a}(z)}{i a}=f_{a}^{\prime}(z)+\frac{1}{i a} \int_{z-i a / 2}^{z+i a / 2} d w\left(f_{a}^{\prime}(w)-f_{a}^{\prime}(z)\right), \quad z \in \mathscr{B}_{0} .
$$

Clearly, the second term on the rhs can be majorized by 


$$
\sup _{w \in\{z+i b \mid b \in[-a / 2, a / 2]\}}\left|f_{a}^{\prime}(w)-f_{a}^{\prime}(z)\right| .
$$

Now $f_{a}^{\prime}(z)$ converges to $f^{\prime}(z)$ uniformly on compacts $K \subset \mathscr{B}_{0}$, and the lhs of $(2.80)$ converges to $-i \chi(z)$ uniformly on $K$ [due to (2.74)], so one easily deduces (2.77).

We conclude this subsection with some comments on the assumptions of the theorem just obtained. In later applications, the assumptions on $\phi_{a}(z)$ are easily verified. Moreover, fixing $z$ $\in \mathscr{C}_{0}$, the function $\phi_{a}(z)$ is actually real-analytic in $a$ for $a \in \mathbb{R}$. (Note this property is stronger than (2.74).) Possibly, these properties already entail the hypotheses (2.75) and (2.76), but we believe this is not true in general. (Observe that the function $f_{a}(z)$ is not likely to be analytic at $a=0$ for $z \in \mathscr{B}_{0}$.)

The above convergence result should also be compared to the last assertion of Theorem II.2. Taking $\phi_{a}(z) \equiv a \phi(z)$, one sees that this assertion amounts to a simple special case of Theorem II.4-except that the analyticity region is different, and that the constant left undetermined in $f(z)=-i \psi(z)$ by (2.77) is fixed in terms of $\chi(z)=\phi(z)$. In this connection we point out that the choice of the region $\mathscr{B}_{0}(2.73)$ in which $\phi_{a}(z)$ is assumed to remain analytic as $a \rightarrow 0$ is determined more by convenience of exposition than by necessity. Indeed, as will be exemplified by Prop. III.7 below, the maximal region with this property can be larger, and correspondingly one can obtain convergence in this larger region.

\section{Fourier series solutions}

We proceed by obtaining results that will enable us to solve A $\Delta$ Es occurring in the trigonometric and elliptic contexts. Correspondingly, we will be dealing with meromorphic functions that are periodic in the real direction. It is convenient to parametrize this period by $\pi / r, r \in(0, \infty)$. For $\Psi(x) \in L^{2}([-\pi / 2 r, \pi / 2 r], d x)$ we employ Fourier coefficients

$$
\hat{\Psi}_{n} \equiv \frac{r}{\pi} \int_{-\pi / 2 r}^{\pi / 2 r} d x \Psi(x) e^{2 i n r x}, \quad n \in \mathbb{Z}
$$

so that

$$
\Psi(x)=\sum_{n \in \mathbb{Z}} \hat{\Psi}_{n} e^{-2 i n r x}
$$

with the series converging in the $L^{2}$-topology.

As we have seen in the previous subsection, the $\mathrm{A} \Delta \mathrm{E}(2.22)$ naturally leads to hyperbolic functions when $\phi$ satisfies (2.18)-(2.21), cf. (2.26) and (2.27). In much the same way, periodicity of $\phi(z)$ leads to the emergence of elliptic functions. It is convenient to collect some features of the functions that arise before stating the analog of Theorem II.2. First, we recall the product representations of the Weierstrass $\sigma$-function (cf., e.g., Ref. 16): We have, taking $r, a>0$,

$$
\sigma\left(z ; \frac{\pi}{2 r}, \frac{i a}{2}\right)=\exp \left(\eta z^{2} r / \pi\right) \frac{\sin r z}{r} \prod_{k=1}^{\infty} \frac{\left(1-p^{k} \exp (2 i r z)\right)(z \rightarrow-z)}{\left(1-p^{k}\right)^{2}}
$$

with

$$
p \equiv \exp (-2 a r)
$$

or, alternatively,

$$
\sigma\left(z ; \frac{\pi}{2 r}, \frac{i a}{2}\right)=\exp \left(\eta^{\prime} z^{2} / i a\right) \frac{\operatorname{sh} \pi z / a}{\pi / a} \prod_{k=1}^{\infty} \frac{\left(1-\vec{p}^{k} \exp (2 \pi z / a)\right)(z \rightarrow-z)}{\left(1-\bar{p}^{k}\right)^{2}}
$$


with

$$
\tilde{p} \equiv \exp \left(-2 \pi^{2} / a r\right)
$$

Here, $\eta$ and $\eta^{\prime}$ are connected by Legendre's relation

$$
\eta^{\prime}=i \eta \operatorname{\eta r} / \pi-i r
$$

The function

$$
s(r, a ; z) \equiv \sigma\left(z ; \frac{\pi}{2 r}, \frac{i a}{2}\right) \exp \left(-\eta z^{2} r / \pi\right)
$$

plays a key role in the sequel. In view of (2.84) $s$ is odd and $\pi / r$-antiperiodic, and satisfies

$$
\lim _{a \rightarrow \infty} s(r, a ; z)=\frac{\sin r z}{r} \quad \text { (uniformly on compacts). }
$$

Moreover, using (2.86) and (2.88) one sees that $s$ solves the $\mathrm{A} \Delta \mathrm{E}$

$$
\frac{s(z+i a / 2)}{s(z-i a / 2)}=-\exp (-2 i r z)
$$

and obeys

$$
\lim _{r \rightarrow 0} s(r, a ; z)=\frac{\operatorname{sh} \pi z / a}{\pi / a} \quad \text { (uniformly on compacts) }
$$

Note that $s(r, a ; z)$ is not a regular solution to the regular $\mathrm{A} \Delta \mathrm{E}(2.91)$ : It has zeros for $\operatorname{Im} z=0$.

Next, using the power series for $\ln (1-x),|x|<1$, one easily verifies the identity

$$
\prod_{k=1}^{\infty}\left(1-p^{k} \exp (2 i r z)\right)(z \rightarrow-z)=\exp \left(-\sum_{n=1}^{\infty} \frac{e^{-n r a}}{n \operatorname{shn} n r a} \cos 2 n r z\right), \quad|\operatorname{Im} z|<a .
$$

Combining this with (2.84) and (2.89) one obtains

$$
s(r, a ; z)=\frac{\sin r z}{r} \exp \left(\sum_{n=1}^{\infty} \frac{e^{-n r a}}{n \operatorname{sh} n r a}(1-\cos 2 n r z)\right), \quad|\operatorname{Im} z|<a .
$$

From this representation we deduce

$$
\frac{s^{\prime}(r, a ; z)}{s(r, a ; z)}=r \cot r z+2 r \sum_{n=1}^{\infty} \frac{e^{-n r a}}{\operatorname{sh} n r a} \sin 2 n r z, \quad|\operatorname{Im} z|<a .
$$

Using the elementary Fourier series

$$
\operatorname{cotr}(z+i a / 2)=-i-2 i \sum_{n=1}^{\infty} e^{-n r a} e^{2 i n r z}, \quad \operatorname{Im} z>-a / 2
$$

we finally obtain 


$$
K(r, a ; z)=i r+i r \sum_{n \in \mathbb{Z}^{*}} \frac{e^{-2 i n r z}}{\operatorname{sh} n r a}, \quad|\operatorname{Im} z|<a / 2,
$$

where we have introduced

$$
K(r, a ; z) \equiv \frac{d}{d z} \ln s(r, a ; z+i a / 2)
$$

Note that (2.92) entails

$$
\lim _{r \rightarrow 0} K(r, a ; z)=\frac{\pi}{a} \operatorname{th} \frac{\pi z}{a}
$$

uniformly on compact subsets of $|\operatorname{Im} z|<a / 2$.

Theorem II.5: Assume $\phi(z)$ is a function with the following properties:

$$
\begin{gathered}
\phi(z) \text { is analytic in a strip }|\operatorname{Im} z|<s, s \in(0, \infty), \\
\phi(z) \text { has period } \pi / r
\end{gathered}
$$$$
\hat{\phi}_{0}=0 \text {. }
$$

Then the $A \Delta E$ (2.22) has a unique solution $f(a ; z)$ such that

$$
\begin{aligned}
& f(a ; z) \text { is analytic in the strip }|\operatorname{Im} z|<s+a / 2, \\
& \qquad \begin{array}{l}
f(a ; z) \text { has period } \pi / r \\
\hat{f}_{0}=0 .
\end{array}
\end{aligned}
$$

Explicitly, this solution can be written as

$$
f(a ; z)=\frac{1}{2} \sum_{n \in \mathbb{Z}^{*}} \frac{\hat{\phi}_{n} e^{-2 i n r z}}{\operatorname{sh} n r a}, \quad|\operatorname{Im} z| \leqslant a / 2,
$$

or as

$$
f(a ; z)=\frac{1}{2 i \pi} \int_{-\pi / 2 r}^{\pi / 2 r} d u \phi(u) K(r, a ; z-u), \quad|\operatorname{Im} z|<a / 2
$$

It obeys the addition formula (2.28). If $\phi(z)$ is even/odd, then $f(a ; z)$ is odd/even. Finally, the limit relation (2.30) holds true uniformly on compact subsets of the strip $|\operatorname{Imz}|<s$, with $\psi(x)$ the primitive of $\phi(x)$ that satisfies $\hat{\psi}_{0}=0$.

Proof: In order to prove uniqueness, we argue as in the proof of Theorem II. 2 to conclude that the difference $d(z)$ of two solutions satisfying (2.103)-(2.105) extends to an ia-periodic entire function. Since $d(z)$ has period $\pi / r$, too, we deduce that $d(z)$ equals a constant $d$. Now we have $0=\hat{d}_{0}=\pi d / r$ by $(2.105)$, and so uniqueness follows.

Next, we define a function $f(z)$ by the rhs of (2.106). Clearly, $f(z)$ is analytic in $|\operatorname{Im} z|<a / 2$ and has properties (2.104) and (2.105). Moreover, the functions 


$$
b_{ \pm}(x) \equiv \frac{1}{2} \sum_{n \in Z^{*}} \frac{\hat{\phi}_{n} e^{ \pm n r a}}{\operatorname{sh} n r a} e^{-2 i n r x}, \quad x \in \mathbb{R},
$$

are smooth and $\pi / r$-periodic, and (2.33) holds true uniformly on $\mathbb{R}$. (Note that the Fourier coefficients $\hat{\phi}_{n}$ form a fast decreasing sequence, since $\phi(x)$ is real-analytic and $\pi / r$-periodic.) Since we also have

$$
b_{+}(x)-b_{-}(x)=\sum_{n \in \mathbb{Z}^{*}} \hat{\phi}_{n} e^{-2 i n r x}=\phi(x),
$$

the reasoning in the proof of Theorem II.2 can be repeated, showing that $f(z)$ solves (2.22) and has property (2.103).

The representation (2.107) follows from (2.106) and the Fourier series (2.97) by using the Plancherel relations and (2.102). The addition formula (2.28) follows in the same way as in the proof of Theorem II.2. The parity claim is obvious from either (2.106) or (2.107). Using (2.106) with $z \in \mathbb{R}$, it follows from routine arguments that

$$
\operatorname{limiaf}_{a \rightarrow 0}(a ; x)=\sum_{n \in \mathbb{Z}^{*}} \frac{\hat{\phi}_{n} e^{-2 i n r x}}{-2 i n r} \equiv \psi(x), \quad x \in \mathbb{R},
$$

and that $\psi(x)$ is a primitive of $\phi(x)$ with $\hat{\psi}_{0}=0$. The uniform convergence assertion then follows in the same way as before from Vitali's theorem.

Recalling the limit (2.99), one sees that the representation (2.107) turns into (2.27) for $r \rightarrow 0$. More precisely, this holds true for functions $\phi(r ; u)$ with a suitable dependence on $r$. Clearly, one needs some restrictions on this dependence to ensure uniform convergence for $z$ in compacts of the strip $|\operatorname{Im} z|<a / 2$ (say), but we shall not pursue this. (For an explicit example, see Prop. III. 12 in Subsection III B.)

We continue with an analog of Theorem II.3.

Theorem II.6: With (2.18)-(2.21) replaced by (2.100)-(2.102) and (2.44) replaced by

$$
e(z) \equiv \frac{1}{2} \sum_{n \in Z^{*}} \frac{\hat{\phi}_{n}}{\operatorname{sh} n r a}(-2 i n r)^{-k}\left(e^{-2 i n r z}-\sum_{j=0}^{k-1} \frac{(-2 i n r z)^{j}}{j !}\right), \quad|\operatorname{Im} z| \leqslant a / 2,
$$

the assertions of Theorem II.3 hold true.

Proof: With Theorem II.2 replaced by Theorem II.5, and (2.47) by

$$
f_{k}(z) \equiv \frac{1}{2} \sum_{n \in Z^{*}} \frac{\hat{\phi}_{n}}{\operatorname{shn} r a} e^{-2 i n r z}, \quad|\operatorname{Im} z| \leqslant a / 2,
$$

the reasoning in the proof of Theorem II.3 applies verbatim; note that boundedness of $f_{k}(z)$ in the strip $|\operatorname{Im} z| \leqslant a / 2$ entails polynomial boundedness of $f_{l}(z)$ in this strip.

We conclude this subsection with a result pertaining to $\mathrm{A} \Delta \mathrm{Es}(2.72)$, adapting the assumptions of the previous subsection to the periodic context. Thus, for any $a \in\left(0, a_{0}\right]$ the right-hand side $\phi_{a}(z)$ is assumed to satisfy $(2.100)-(2.102)$ and to be analytic in the open period strip

$$
\mathscr{B}_{r} \equiv\{z \in \mathbb{C} \mid \operatorname{Re} z \in(0, \pi / r)\}
$$

Furthermore, the bound (2.74) is assumed to be valid for any compact $K \subset \mathscr{R}_{r}$, with $\chi(z)$ analytic in $\mathscr{R}_{r}$.

Denoting by $f_{a}(z)$ the unique solution to $(2.72)$ given by Theorem II.5, we are prepared to state the analog of Theorem II.4. 
Theorem II.7: Assume in addition to the above that (2.75) holds true for any $\delta \in(0, \pi / r)$ and $M \in(\delta, \pi / r)$, and that the pointwise limit (2.76) exists for any $z \in(0, \pi / r)$. Then the assertions of Theorem II.4 hold true, with $\mathscr{B}_{0}$ replaced by $\mathscr{B}_{r}$.

Proof: Taking $M<\pi / r$ in (2.79) and replacing $\mathscr{B}_{0}$ by $\mathscr{B}_{r}$, the proof of Theorem II.4 applies verbatim.

The comments after Theorem II.4 apply with obvious changes to Theorem II.7, so we shall not spell them out again.

\section{GENERALIZED GAMMA FUNCTIONS}

\section{A. The hyperbolic case}

Consider the integral

$$
\int_{0}^{\infty} \frac{d y}{y}\left(\frac{\sin 2 y z}{2 \operatorname{sh} a_{+} y \operatorname{sh} a_{-} y}-\frac{z}{a_{+} a_{-} y}\right) \equiv g\left(a_{+}, a_{-} ; z\right),
$$

where we take $a_{\delta} \in(0, \infty), \delta=+,-$, until further notice. Obviously, this integral converges absolutely provided $z$ belongs to the strip

$$
S \equiv\left\{z \in \mathbb{C}|| \operatorname{Im} z \mid<\left(a_{+}+a_{-}\right) / 2\right\},
$$

and it defines a function $g$ that is analytic in $S$. In this subsection we study the function

$$
G(z) \equiv \exp (i g(z))
$$

in considerable detail. (Here and in the sequel, we suppress the dependence on $a_{+}, a_{-}$whenever this causes no confusion.) We shall collect our results in propositions that concern various features of $G(z)$.

Proposition III.1 (defining $\mathbf{A} \Delta \mathbf{E s})$ : The function $G(z)$ is analytic and has no zeros in the strip S. It extends to a meromorphic function that is a minimal solution to the three ADEs

$$
\frac{G\left(z+i a_{\delta} / 2\right)}{G\left(z-i a_{\delta} / 2\right)}=2 \operatorname{ch}\left(\pi z / a_{-\delta}\right), \quad \delta=+,-
$$

and

$$
\frac{G\left(z+i\left(a_{+}-a_{-}\right) / 2\right)}{G\left(z-i\left(a_{+}-a_{-}\right) / 2\right)}=\frac{\operatorname{sh}\left(\pi z / a_{-}\right)}{\operatorname{sh}\left(\pi z / a_{+}\right)} .
$$

It is the unique minimal solution satisfying

$$
G(0)=1, \quad|G(x)|=1, \quad x \in \mathbb{R} .
$$

Proof: The first assertion is clear from (3.1)-(3.3). Taking $\delta=+$ in (3.4) and denoting the rhs by $\Phi(z)$, the assumptions of Theorem II.3 are satisfied, with $a=a_{+}, s=a_{-} / 2$ and $k=3$. Indeed, we have

$$
\phi(z) \equiv\left(\frac{d}{d z}\right)^{3} \ln \Phi(z)=\frac{\pi}{a_{-}}\left(\frac{d}{d z}\right)^{2} \operatorname{th}\left(\pi z / a_{-}\right)
$$

so that (cf. (217)) 


$$
\hat{\phi}(y)=\frac{-i y^{2}}{2 \operatorname{sh}\left(a_{-} y / 2\right)} .
$$

From this the properties (2.18)-(2.21) are evident.

As a consequence the $\mathrm{A} \Delta \mathrm{E}$ at hand admits minimal solutions; these can be written as (2.43)(2.44) with $k=3$ and

$$
\begin{aligned}
e(z) & =-\frac{1}{4} \int_{-\infty}^{\infty} \frac{d y}{y \operatorname{sh} a_{+} y \operatorname{sh} a_{-} y}\left(e^{-2 i y z}-\left(1-2 i y z-2 y^{2} z^{2}\right)\right) \\
& =i \int_{0}^{\infty} \frac{d y}{\operatorname{sh} a_{+} y \operatorname{sh} a_{-} y}\left(\frac{\sin 2 y z}{2 y}-z\right) .
\end{aligned}
$$

To determine $c_{1}, c_{2}, c_{3}$ we follow the proof of Theorem II.3. Thus, we start from

$$
f_{3}(z)=-4 i \int_{0}^{\infty} d y y^{2} \cos (2 y z) / \operatorname{sh} a_{+} y \operatorname{sh} a_{-} y,
$$

cf. (2.47). Then we get

$$
\int_{0}^{z} d s f_{3}(s)=-2 i \int_{0}^{\infty} d y y \sin (2 y z) / \operatorname{sh} a_{+} y \operatorname{sh} a_{-} y
$$

so that

$$
\int_{-i a_{+} / 2}^{i a_{+} / 2} d s f_{3}(s)=4 \int_{0}^{\infty} d y y / \operatorname{sh} a_{-} y=\left(\frac{\pi}{a_{-}}\right)^{2} .
$$

From (2.50) we then have $c_{3}=0$, and so

$$
f_{2}(z)=-2 i \int_{0}^{\infty} d y y \sin (2 y z) / \operatorname{sh} a_{+} y \operatorname{sh} a_{-} y .
$$

Now $f_{2}(z)$ is odd, so (2.52) yields $c_{2}=0$. Hence,

$$
f_{1}(z)=i \int_{0}^{\infty} d y(\cos (2 y z)-1) / \operatorname{sh} a_{+} y \operatorname{sh} a_{-} y,
$$

cf. (2.51), so that

$$
\int_{0}^{z} d s f_{1}(s)=i \int_{0}^{\infty} d y\left(\frac{\sin 2 y z}{2 y}-z\right) / \operatorname{sh} a_{+} y \operatorname{sh} a_{-} y=e(z),
$$

cf. (3.9). Now we have

$$
\pm e\left( \pm i a_{+} / 2\right)=\frac{1}{2} \int_{0}^{\infty} d y\left(\frac{a_{+}}{\operatorname{sh} a_{+} y \operatorname{sh} a_{-} y}-\frac{1}{y \operatorname{sh} a_{-} y}\right) .
$$

Also, recalling (A33) and (A34), we may write 


$$
\ln 2=\int_{0}^{\infty} d y\left(\frac{1}{a_{-} y^{2}}-\frac{1}{y \operatorname{sh} a_{-} y}\right) .
$$

Using (2.52) once more, we obtain

$$
c_{1}=\left(i a_{+}\right)^{-1}\left(\ln 2-e\left(i a_{+} / 2\right)+e\left(-i a_{+} / 2\right)\right)=i \int_{0}^{\infty} d y\left(\frac{1}{\operatorname{sh} a_{+} y \operatorname{sh} a_{-} y}-\frac{1}{a_{+} a_{-} y^{2}}\right) .
$$

Combining (2.51) with (3.15) now yields

$$
f_{0}(z)=c_{1} z+e(z)=i g\left(a_{+}, a_{-} ; z\right),
$$

cf. (3.1). In view of (3.3), this entails that $G(z)$ solves (3.4) with $\delta=+$. Since the function $G$ is manifestly symmetric in $a_{+}, a_{-}$, it solves (3.4) with $\delta=-$, too.

To prove that $G$ also satisfies the $\mathrm{A} \Delta \mathrm{E}$ (3.5), we observe that we may write

$$
\frac{G\left(z+i\left(a_{+}-a_{-}\right) / 2\right)}{G\left(z-i\left(a_{+}-a_{-}\right) / 2\right)}=\frac{G\left(z-i a_{-} / 2+i a_{+} / 2\right)}{G\left(z-i a_{-} / 2-i a_{+} / 2\right)} \cdot \frac{G\left(z-i a_{+} / 2-i a_{-} / 2\right)}{G\left(z-i a_{+} / 2+i a_{-} / 2\right)} .
$$

From (3.4) we now deduce that (3.5) holds true. Finally, the uniqueness assertion is clear from Theorem II.1.

We point out that the identity (2.71) can also be obtained from the $\mathrm{A} \Delta \mathrm{E}$ (3.5). Similarly, the proposition entails the identity

$$
\int_{0}^{\infty} \frac{d y}{y}\left(\frac{1}{a y}-\frac{\cos 2 y z}{\operatorname{sh} a y}\right)=\ln \left(2 \operatorname{ch} \frac{\pi z}{a}\right), \quad a>0, \quad|\operatorname{Im} z|<a / 2 .
$$

Indeed, this identity amounts to the function ig [as given by (3.1)] satisfying the additive versions of the $\mathrm{A} \Delta \mathrm{Es}$ (3.4). The integral (3.21) can also be derived directly from (A33), (A34) and (2.17). In this way one can obtain a shorter proof of (3.4). The above proof, however, shows how the function $G(z)$ emerges from the general theory presented in Subsection II B, when one takes one of the $\mathrm{A} \Delta \mathrm{Es}$ (3.4) as a starting point.

Proposition III.2 (automorphy properties): One has

$$
\begin{gathered}
G(-z)=1 / G(z), \\
G\left(a_{-}, a_{+} ; z\right)=G\left(a_{+}, a_{-} ; z\right), \\
G\left(\lambda a_{+}, \lambda a_{-} ; \lambda z\right)=G\left(a_{+}, a_{-} ; z\right), \quad \lambda \in(0, \infty) .
\end{gathered}
$$

For any $M, N \in \mathbb{N}^{*}$ one has the multiplication formula

$$
G\left(\frac{a_{+}}{M}, \frac{a_{-}}{N} ; z\right)=\prod_{j=1}^{M} \prod_{k=1}^{N} G\left(a_{+}, a_{-} ; z+\frac{i a_{+}}{2 M}(M+1-2 j)+\frac{i a_{-}}{2 N}(N+1-2 k)\right) .
$$

Proof: All of these properties readily follow from the integral representation (3.1)-(3.3) and meromorphy of $G$. Indeed, the first three are immediate from (3.1). Taking first $N=1$ in (3.25), and using (3.1) and the identity (2.38) to rewrite the rhs, one obtains the desired result for $G\left(a_{+} / M, a_{-} ; z\right)$; the general case then follows by using (3.23).

Note that when one takes $M=N$ in the formula (3.25), one can use (3.24) to write its lhs as $G\left(a_{+}, a_{-} ; N z\right)$.

Proposition III.3 (zeros, poles, residues): The zeros and poles of $G(z)$ are given by 


$$
\begin{gathered}
z_{k l}^{+} \equiv i\left(a_{+}(k+1 / 2)+a_{-}(l+1 / 2)\right), \quad k, l \in \mathbb{N} \quad \text { (zeros) } \\
z_{k l}^{-} \equiv-z_{k l}^{+}, \quad k, l \in \mathbb{N} \quad \text { (poles) }
\end{gathered}
$$

For a given $\left(k_{0}, l_{0}\right) \in \mathbb{N}^{2}$, the multiplicities of the pole $z_{k_{0} l_{0}}^{-}$and zero $z_{k_{0} l_{0}}^{+}$are equal to the number of distinct pairs $(k, l) \in \mathbb{N}^{2}$ such that $z_{k l}^{+}=z_{k_{0} l_{0}}^{+}$; in particular, for $a_{+} / a_{-} \notin Q$ all poles and zeros are simple. The pole at $z_{00}^{-}$is simple and has residue

$$
r_{00}=\frac{i}{2 \pi}\left(a_{+} a_{-}\right)^{1 / 2}
$$

More generally, if the quantity

$$
t_{k l} \equiv \prod_{m=1}^{k} \sin \left(\pi m a_{+} / a_{-}\right) \prod_{n=1}^{l} \sin \left(\pi n a_{-} / a_{+}\right)
$$

is non-zero, then the pole at $z_{k l}^{-}$is simple and has residue

$$
r_{k l}=(-)^{k l}(-1 / 2)^{k+l} r_{00} / t_{k l}
$$

Conversely, if $z_{k l}^{-}$is a simple pole, then one has $t_{k l} \neq 0$.

Proof: In view of (3.23), we may assume $a_{+} \leqslant a_{-}$. Iterating the $\mathrm{A} \Delta \mathrm{E}$ (3.4) with $\delta=+$ we obtain

$$
G\left(z-i M a_{+}\right)=P_{M}(z) G(z), \quad M \in \mathbb{N}^{*},
$$

where

$$
P_{M}(z) \equiv\left(\prod_{m=1}^{M} 2 \operatorname{ch} \frac{\pi}{a_{-}}\left(z-i a_{+}(m-1 / 2)\right)\right)^{-1}
$$

Now the poles of $P_{M}(z)$ occur at (and only at)

$$
z_{m l} \equiv i a_{+}(m-1 / 2)-i a_{-}(l+1 / 2), \quad m=1, \ldots, M, \quad l \in \mathbb{Z} .
$$

Introducing the strip

$$
S_{-} \equiv\left\{z \in \mathbb{C} \mid \operatorname{Im} z \in a_{-}[-1 / 2,1 / 2)\right\}
$$

and fixing $m \in\{1, \ldots, M\}$, there exists a unique $l \geqslant 0$ such that $z_{m l} \in S_{-}$. Since $G(z)$ is analytic and non-zero in $S_{-}$, it now follows from (3.31) that $G$ has $M$ and only $M$ poles (counting multiplicity) in the shifted strip $S_{-}-i M a_{+}$; these occur at $z_{k l}^{-}, k=0, \ldots, M-1$, with $l \in \mathbb{N}$ uniquely determined by $k$ and $M$.

Now for a given pair $\left(k_{0}, l_{0}\right) \in \mathbb{N}^{2}$ one can find some $M_{0}>k_{0}$ such that $\overline{z_{0} l_{0}} \in S_{-}-i M_{0} a_{+}$ (since the shifted strips cover the lower half plane). Also, for any pair $(k, l) \in \mathbb{N}^{2}$ such that $z_{k l}^{-}=z_{k_{0} l_{0}}^{-}$, one must have $k<M_{0}$ (since $z_{k l}^{-} \in S_{-}-i M_{0} a_{+}$entails $a_{+}(k+1 / 2)+a_{-} l \leqslant a_{+} M_{0}$ ). Consequently, the multiplicity of the pole of $P_{M_{0}}(z)$ at $z=z_{k_{0} l_{0}}^{-}+i M_{0} a_{+}$equals the number of pairs satisfying $z_{k l}^{-}=z_{k_{0} l_{0}}^{-}$.

The upshot is that the poles of $G\left(a_{+}, a_{-} ; z\right)$ in the lower half plane are given by (3.27) and have the asserted multiplicity. Since $G$ is non-zero in $S_{-}$and $P_{M}$ has no zeros at all, it follows from (3.31) that $G$ is non-zero in the lower half plane. Recalling (3.22), the first two assertions easily follow. 
To prove the third one, we use (3.4) with $\delta=+$ to get

$$
G\left(z-i\left(a_{+}+a_{-}\right) / 2\right)=\left(-2 i \operatorname{sh} \frac{\pi z}{a_{-}}\right)^{-1} G\left(z+i\left(a_{+}-a_{-}\right) / 2\right) .
$$

From this we read off

$$
r_{00}=\frac{i a_{-}}{2 \pi} G\left(i\left(a_{+}-a_{-}\right) / 2\right)
$$

Similarly, using (3.4) with $\delta=-$ we obtain

$$
r_{00}=\frac{i a_{+}}{2 \pi} G\left(i\left(a_{-}-a_{+}\right) / 2\right)
$$

Combining these two expressions for $r_{00}$ with (3.22), we deduce

$$
G\left(i\left(a_{+}-a_{-}\right) / 2\right)=\left(a_{+} / a_{-}\right)^{1 / 2},
$$

and so (3.28) follows. (Note that (3.1) and (3.3) entail that $G$ is positive for $z \in i\left(a_{+}+a_{-}\right)$ $\times(-1 / 2,1 / 2)$. Note also that (3.38) can be derived from (3.5).)

Finally, we exploit both $\mathrm{A} \Delta \mathrm{Es}$ (3.4) to write

$$
G\left(z+z_{k l}\right)=(-)^{k l+k+l}\left(\prod_{m=1}^{k} 2 i \operatorname{sh} \frac{\pi}{a_{-}}\left(z-i m a_{+}\right) \prod_{n=1}^{l} 2 i \operatorname{sh} \frac{\pi}{a_{+}}\left(z-i n a_{-}\right)\right)^{-1} G\left(z+z_{00}\right) .
$$

Taking $z \rightarrow 0$ in this identity, the remaining assertions follow.

In principle, the residue at $z_{k_{0} l_{0}}^{-}$can still be determined by using (3.30) even when ${\overline{k_{0} l_{0}}}^{-}$is not a simple pole. Indeed, in that case one must have $a_{+} / a_{-} \in \mathbb{Q}$; choosing sequences $a_{\delta, n} \rightarrow a_{\delta}, \delta=+,-$, for $n \rightarrow \infty$ such that $a_{+, n} / a_{-, n} \notin \mathbb{Q}$, the residue equals the limit of the sum of the residues at the simple poles that coalesce at $z_{k_{0} l_{0}}^{-}$. There is presumably an explicit formula for the limit, but we have not pursued this.

It is evident from (3.3) and the above that $g(z)$ extends from an analytic function in $S$ to a multi-valued function with logarithmic branch points at (3.26) and (3.27). It is convenient to specialize to the branch obtained by restricting $z$ to the cut plane $\mathbb{C}\left(a_{+}+a_{-}\right)$, where

$$
\mathbb{C}(d) \equiv \mathrm{C} \backslash\{ \pm i[d / 2, \infty)\}, \quad d>0 .
$$

This branch will be again denoted $g(z)$. Asymptotic properties for $\operatorname{Re} z \rightarrow \pm \infty$ are most easily obtained for the special case $a_{+}=a_{-} \equiv a$; the general case can then be handled by a comparison argument, cf. Prop. III.4 below.

We start from the identity

$$
g(a, a ; z)=-\frac{1}{\pi} b(\pi z / a)
$$

where we have introduced

$$
b(w) \equiv \int_{0}^{w} d t t \operatorname{cth} t, \quad w \in \mathbb{C}(2 \pi) .
$$


(To see that this holds true, use (3.1) on the lhs and take $z$-derivatives; this yields a linear combination of the identities (2.66) and (2.69).) Next, we write $\operatorname{ch} t=\operatorname{sh} t+e^{-t}$ to obtain

$$
b(w)=w^{2} / 2+c_{+}-b_{+}(w), \quad \operatorname{Re} w>0,
$$

where

$$
\begin{gathered}
b_{+}(w) \equiv \int_{w}^{\infty} d t \frac{t e^{-t}}{\operatorname{sh} t}, \quad \operatorname{Re} w>0, \\
c_{+} \equiv \int_{0}^{\infty} d t \frac{t e^{-t}}{\operatorname{sh} t}=\frac{F_{1}^{\prime}(0)}{2 i}=\sum_{m=1}^{\infty} \frac{1}{2 m^{2}}=\frac{\pi^{2}}{12},
\end{gathered}
$$

cf. (A8) and (A10). From this representation we read off the bounds

$$
\begin{gathered}
b(w)=\frac{w^{2}}{2}+\frac{\pi^{2}}{12}+O(\exp ((\epsilon-2) w)), \quad \operatorname{Re} w \rightarrow \infty, \\
b^{\prime}(w)=w+O(\exp ((\epsilon-2) w)), \quad \operatorname{Re} w \rightarrow \infty .
\end{gathered}
$$

Here, $\epsilon$ is a fixed positive number and the bounds hold true uniformly for $\operatorname{Im} w$ varying over compact subsets of $\mathbb{R}$.

Of course, these bounds entail bounds on $g(a, a ; z)$ via (3.41). More generally, they can be exploited to derive bounds on $g\left(a_{+}, a_{-} ; z\right)$, as will now be detailed.

Proposition III.4 (asymptotics): Fixing $\epsilon>0$ and setting

$$
a_{m} \equiv \max \left(a_{+}, a_{-}\right)
$$

one has

$$
\begin{gathered}
\pm g\left(a_{+}, a_{-} ; z\right)=-\frac{\pi z^{2}}{2 a_{+} a_{-}}-\frac{\pi}{24}\left(\frac{a_{+}}{a_{-}}+\frac{a_{-}}{a_{+}}\right)+O\left(\exp \left( \pm\left(\epsilon-2 \pi / a_{m}\right) z\right)\right), \quad \operatorname{Re} z \rightarrow \pm \infty, \\
\pm g^{\prime}\left(a_{+}, a_{-} ; z\right)=-\frac{\pi z}{a_{+} a_{-}}+O\left(\exp \left( \pm\left(\epsilon-2 \pi / a_{m}\right) z\right)\right), \quad \operatorname{Re} z \rightarrow \pm \infty,
\end{gathered}
$$

where the bounds are uniform for $\operatorname{Im} z$ in $\mathbb{R}$-compacts.

Proof: Since $g$ is odd in $z$, it suffices to verify the $\operatorname{Re} z \rightarrow \infty$ asymptotics. Now when $a_{+}=a_{-}$, the formulas (3.49) and (3.50) are immediate from (3.41), and (3.46) and (3.47), resp. Since $g$ is symmetric in $a_{+}, a_{-}$, it remains to consider the case $a_{+}<a_{-}$.

To this end we rewrite (3.1) as

$$
a_{+} a_{-} g\left(a_{+}, a_{-} ; z\right)=a^{2} g(a, a ; z)+d(z)
$$

where we have introduced

$$
\begin{gathered}
a \equiv\left(\frac{a_{+}^{2}+a_{-}^{2}}{2}\right)^{1 / 2}, \\
d(z) \equiv \int_{0}^{\infty} d y I(y) \sin 2 y z,
\end{gathered}
$$


with

$$
I(y) \equiv \frac{1}{2 y}\left(\frac{a_{+} a_{-}}{\operatorname{sh} a_{+} y \operatorname{sh} a_{-} y}-\frac{a^{2}}{\operatorname{sh}^{2} a y}\right) .
$$

Here, we take $z$ in the strip $S(3.2)$, so that the integral converges (note $a_{+}+a_{-} \leqslant 2 a$ ). Now we have

$$
I(y)=c\left(a_{+}, a_{-}\right) y+O\left(y^{3}\right), \quad y \rightarrow 0,
$$

so $I(y)$ is analytic in the strip $|\operatorname{Im} y|<\pi / a_{-}$. Hence, fixing $z \in S$ and $r \in\left(0, \pi / a_{-}\right)$, we may shift contours to obtain

$$
2 i d(z)=e^{-2 r z} \int_{-\infty}^{\infty} d u I(u+i r) e^{2 i u z}
$$

From this we deduce that $d(z)$ and $d^{\prime}(z)$ are $O\left(e^{-2 r z}\right)$ for $\operatorname{Re} z \rightarrow \infty$, uniformly for $z$ in a closed substrip of $S$.

Combining these bounds with (3.51) and the $\operatorname{Re} z \rightarrow \infty$ asymptotics of $g(a, a ; z)$, we deduce that (3.49) and (3.50) hold true uniformly for $z$ in the strip $|\operatorname{Im} z| \leqslant a_{+}$. Finally, we exploit the $\mathrm{A} \Delta \mathrm{Es}$

$$
g\left(z \pm i a_{+}\right)=g(z) \mp i \ln \left(2 \operatorname{ch} \frac{\pi}{a_{-}}\left(z \pm i a_{+} / 2\right)\right)
$$

to infer that the bounds hold uniformly for $|\operatorname{Im} z| \leqslant 2 a_{+} ;$by iteration, the proposition now follows.

Thus far, we have taken $a_{+}$and $a_{-}$positive. However, fixing $z \in \mathbb{R}$, it is already obvious from (3.1) that $G\left(a_{+}, a_{-} ; z\right)$ extends to a function that is analytic and non-zero for $a_{+}, a_{-}$in the (open) right half plane. Note this is consistent with the analytic continuation of (3.26) and (3.27): The imaginary part of the rhs is non-zero for $a_{+}, a_{-}$in the right half plane.

More generally, we shall now prove that $G$ can be continued to a function that is meromorphic in $a_{+}, a_{-}$and $z$, provided the ratio variable

$$
\rho \equiv a_{-} / a_{+}
$$

stays away from the negative real axis. To this end we consider the auxiliary function

$$
A(\rho, \lambda) \equiv \prod_{j=0}^{\infty} F((j+1 / 2) \rho, \lambda), \quad \rho \in \mathbb{C}^{-}, \quad \lambda \in \mathbb{C},
$$

where $\mathbb{C}^{-}$denotes the cut plane (A15). In view of (B22) and (B19) this is a well-defined meromorphic function in $\mathbb{C}^{-} \times \mathbb{C}$. Moreover, from (A40) we readily deduce

$$
A(\rho, \lambda)=\exp \left(\int_{0}^{\infty} \frac{d t}{t \operatorname{sh}(\rho t / 2)}(2 \lambda-\operatorname{sh}(\lambda t) \operatorname{cth}(t / 2))\right), \quad \rho>0, \quad|\operatorname{Re} \lambda|<\rho .
$$

Now from (3.1) and (3.3) we have 


$$
\begin{aligned}
G\left(z+i a_{+} / 2\right) G\left(z-i a_{+} / 2\right)= & \exp \left(\int_{0}^{\infty} \frac{d t}{t \operatorname{sh}(\rho t / 2)}\left(\operatorname{sh}\left(i t z / a_{+}\right) \operatorname{cth}(t / 2)-\frac{2 i z}{a_{+}}\right)\right) \\
& \times \exp \left(i \int_{0}^{\infty} \frac{d t}{t}\left(\frac{2 z}{a_{+} \operatorname{sh}\left(a_{-} t / 2 a_{+}\right)}-\frac{4 z}{a_{-} t}\right)\right) \\
= & A\left(\rho,-i z / a_{+}\right) \exp \left(-\frac{2 i z}{a_{+}} \ln 2\right),
\end{aligned}
$$

where we used (A33) and (A34). Next, we introduce the new variable

$$
\lambda \equiv-i z / a_{+}
$$

and combine (3.61) and the $\mathrm{A} \Delta \mathrm{E}(3.4)$ to deduce

$$
G\left(a_{+}, a_{-} ; z+i a_{+} / 2\right)^{2}=A(\rho, \lambda) \exp (2 \lambda \ln 2) \cdot 2 \cos (\pi \lambda / \rho) .
$$

We are now prepared for the following proposition.

Proposition III.5 (meromorphic continuation): The function $G\left(a_{+}, a_{-} ; z\right)$ admits analytic continuation to a function that is meromorphic in $a_{+}, a_{-}$and $z$, provided $\rho \equiv a_{-} / a_{+}$stays in $\mathrm{C}^{-}$. Fixing $a_{+}, a_{-}$with $\operatorname{Im} \rho \neq 0$, one obtains a meromorphic function whose zeros and poles are simple and located at (3.26) and (3.27), resp.

Proof: The function

$$
B(\rho, \lambda) \equiv A(\rho, \lambda) \cos (\pi \lambda / \rho)
$$

is meromorphic in $\mathrm{C}^{-} \times \mathrm{C}$, so in view of (3.63) we need only show that for $\rho \notin \mathbb{R}$ all of its zeros and poles are double and located at

$$
\begin{gathered}
\lambda=k+(l+1 / 2) \rho, \quad k, l \in \mathbb{N} \quad(\text { zeros), } \\
\lambda=-k-1-(l+1 / 2) \rho, \quad k, l \in \mathbb{N} \quad \text { (poles). }
\end{gathered}
$$

Recalling the definitions (2.59) and (A39), we obtain the representation

$$
B(\rho, \lambda)=\cos (\pi \lambda / \rho) \prod_{j=0}^{\infty} \frac{\Gamma((j+1 / 2) \rho+\lambda)}{\Gamma((j+1 / 2) \rho-\lambda)} \frac{\Gamma(1+(j+1 / 2) \rho+\lambda)}{\Gamma(1+(j+1 / 2) \rho-\lambda)} \exp (-4 \lambda \ln (j+1 / 2) \rho)
$$

from which these features can be read off.

Of course, the proposition just proved entails that various formulas involving $G$ can be analytically continued. We mention specifically (3.4), (3.5), (3.22)-(3.25) [note one can take $\lambda$ $\in \mathrm{C}^{*}$ in (3.24)], (3.28)-(3.30), and the special values

$$
G\left(i\left(a_{\delta}-a_{-\delta}\right) / 2\right)=\left(a_{\delta} / a_{-\delta}\right)^{1 / 2}, \quad G\left( \pm i a_{\delta} / 2\right)=2^{ \pm 1 / 2}, \quad \delta=+,-
$$

(These values easily follow from (3.1)-(3.5).)

We proceed by detailing the relation to the gamma function. To this end we introduce

$$
H(\rho ; z) \equiv G(1, \rho ; \rho z+i / 2) \exp \left(i z \ln (2 \pi \rho)-2^{-1} \ln (2 \pi)\right), \quad \rho \in \mathbb{C}^{-}, \quad z \in \mathbb{C} .
$$

This renormalized version of $G\left(a_{+}, a_{-} ; z\right)$ is such that the two A $\Delta \mathrm{Es}$ (3.4) translate into the $\mathrm{A} \Delta \mathrm{E}$ 


$$
\frac{H(\rho ; z+i / 2)}{H(\rho ; z-i / 2)}=\frac{i \operatorname{sh} \pi \rho z}{\pi \rho}
$$

and functional equation

$$
H(\rho ; z) H(\rho ;-z)=\frac{\operatorname{ch} \pi z}{\pi}
$$

(Use (3.22) to check (3.71).) We shall now show that the $\rho \rightarrow 0$ limit of $H(\rho ; z)$ exists and equals $1 / \Gamma(i z+1 / 2)$. Accordingly, (3.70) and (3.71) turn into the $\mathrm{A} \Delta \mathrm{E}$ and functional equation satisfied by the gamma function.

Proposition III.6 (relation to gamma function): Taking $\rho \in(0, \infty)$, one has

$$
\lim _{\rho \downarrow 0} H(\rho ; z)=1 / \Gamma(i z+1 / 2)
$$

uniformly for $z$ in C-compacts. More generally, fix $\epsilon \in(0, \infty), \phi \in(0, \pi)$, and an arbitrary compact $K \subset \mathrm{C}$. Then there exists $\delta=\delta(\epsilon, \phi, K) \in(0, \infty)$ such that

$$
|H(\rho ; z) \Gamma(i z+1 / 2)-1|<\epsilon, \quad z \in K, \quad|\operatorname{Arg} \rho| \leqslant \pi-\phi,|\rho| \in(0, \delta] .
$$

Proof: We begin by proving (3.72). Since the function $1 / \Gamma(i z+1 / 2)$ is entire, we need only show

$$
\begin{gathered}
\lim _{\rho \downarrow 0} P(\rho ; z)=1 \quad \text { (uniformly on compacts), } \\
P(\rho ; z) \equiv H(\rho ; z) \Gamma(i z+1 / 2) .
\end{gathered}
$$

Now from Prop. III. 3 we see that the poles of $\Gamma(i z+1 / 2)$ are matched by zeros of $H(\rho ; z)$, so that $P(\rho ; z)$ has no poles and zeros in the strip

$$
S_{\rho} \equiv\{z \in \mathbb{C}|| \operatorname{Im} z \mid<1 / 2+1 / \rho\}
$$

We continue by deriving an integral representation for $P(\rho ; z)$ that holds true in $S_{\rho}$. To this end we first take $|\operatorname{Im} z|<1 / 2$. Then we may use (3.3) and (3.1) to write

$$
G(1, \rho ; \rho z+i / 2)=\exp \left(\int_{0}^{\infty} \frac{d y}{y}\left(\frac{e^{2 i \rho y z} e^{-y}-e^{-2 i \rho y z} e^{y}}{4 \operatorname{sh} y \operatorname{sh} \rho y}-\frac{i z}{y}+\frac{1}{2 \rho y}\right)\right) .
$$

Also, from (A37) we obtain

$$
\frac{\Gamma(i z+1 / 2)}{(2 \pi)^{1 / 2}}=\exp \left(\int_{0}^{\infty} \frac{d y}{y}\left(i z e^{-2 \rho y}-\frac{1}{2 \rho y}+\frac{e^{-2 i \rho y z}\left(e^{y}-e^{-y}\right)}{4 \operatorname{sh} y \operatorname{sh} \rho y}\right)\right) .
$$

Finally, combining (A37) (with $z=1 / 2$ ) and the integral (A29), we write the remaining factor in (3.69) as

$$
\exp (i z \ln (2 \pi \rho))=\exp \left(\int_{0}^{\infty} \frac{d y}{y}\left(\frac{i z}{y}-\frac{i z e^{-y}}{\operatorname{sh} y}-i z e^{-2 \rho y}\right)\right)
$$

Putting the pieces together, we obtain 


$$
P(\rho ; z)=\exp \left(\frac{i}{2} \int_{0}^{\infty} \frac{d y}{y} \frac{e^{-y}}{\operatorname{sh} y \operatorname{sh} \rho y}(\sin (2 \rho y z)-2 z \operatorname{sh} \rho y)\right) .
$$

Clearly, this representation can be analytically continued to the strip $S_{\rho}$, as announced above. Now we fix a compact $K \subset \mathrm{C}$ and note $K \subset S_{\rho}$ for $\rho$ small enough. Rewriting the integral in (3.80) as

$$
\frac{1}{c} \int_{0}^{\infty} d y \frac{e^{-c y}(\sin (2 y z)-2 z \operatorname{sh} y)}{y^{2} \operatorname{sh} y}\left(\frac{c y}{\operatorname{sh} c y}\right), \quad c \equiv 1 / \rho,
$$

it becomes evident that it converges to 0 for $c \rightarrow \infty$ uniformly on $K$. Consequently, we have now proved that (3.72) holds true uniformly on compacts.

To prove the stronger assertion (3.73), we observe that for $z \in K$ and $c>0$ large enough, the contour in (3.81) may be rotated to $e^{i x} y, y \in[0, \infty)$, with $|\chi| \leqslant(\pi-\phi) / 2$, cf. the proof of Theorem B.1. The resulting integral can now be estimated in an obvious way for $c \in \mathbb{C}$ with $|c|$ large enough and $\left|\operatorname{Arg}\left(e^{i \chi} c\right)\right| \leqslant(\pi-\phi) / 2$, and then (3.73) easily follows.

The function $P(\rho ; z)(3.75)$ is of some interest in itself: It is the unique minimal solution to the $\mathrm{A} \Delta \mathrm{E}$

$$
\frac{F(z+i / 2)}{F(z-i / 2)}=\frac{\operatorname{sh} \pi \rho z}{\pi \rho z}
$$

[cf. (3.70)] that satisfies $F(0)=1,|F(x)|=1, x \in \mathbb{R}$. Note that the representation (3.80) can be understood from Theorem II.3.

We conclude this subsection by deriving two more zero step size limits, now involving the function $G(\pi, a ; \cdot)$ for $a \rightarrow 0$. (The choice $a_{+}=\pi$ is notationally convenient; the scaling relation (3.24) can be used for other $a_{+}$-values.) In fact, we shall phrase the limits in terms of the branch $g(z)=-i \ln G(z)$ defined in the cut plane $\mathrm{C}(\pi+a)$, cf. the paragraph containing (3.40). Introducing the functions

$$
\begin{gathered}
d_{a}(\lambda, \mu ; z) \equiv g(\pi, a ; z+i \lambda a)-g(\pi, a ; z+i \mu a), \quad z \in \mathbb{C}(\pi+a), \quad \lambda, \mu \in \mathbb{R}, \\
D_{a}(z) \equiv a g(\pi, a ; z), \quad z \in \mathbb{C}(\pi+a),
\end{gathered}
$$

we are prepared for the following proposition.

Proposition III.7 (zero step size limits): One has

$$
\begin{gathered}
\lim _{a \downarrow 0} d_{a}(\lambda, \mu ; z)=-i(\lambda-\mu) \ln (2 \operatorname{ch} z), \quad \lambda, \mu \in \mathbb{R}, \\
\lim _{a \downarrow 0} D_{a}(z)=-\int_{0}^{z} d w \ln (2 \operatorname{ch} w),
\end{gathered}
$$

uniformly on compact subsets of the cut plane $\mathrm{C}(\pi)$ (3.40). Here, $\ln$ is real-valued for $z$ and $w$ real, resp., and the integration path in (3.86) belongs to $\mathrm{C}(\pi)$.

Proof: From the $\mathrm{A} \triangle \mathrm{E}$ (3.4) with $a_{\delta}=a, a_{-\delta}=\pi$, we deduce that (3.85) need only be proved for $\lambda, \mu \in[-1 / 2,1 / 2]$. Taking from now on $a \in(0, \pi / 4]$ (say), we fix $\lambda$ and $\mu$ in this interval and $z$ in the strip $|\operatorname{Im} z|<\pi / 2$. Then we may use (3.1) to write

$$
d_{a}(\lambda, \mu ; z)=-i \int_{0}^{\infty} \frac{d y}{y}\left(\frac{(\lambda-\mu)}{\pi y}-\frac{\operatorname{sh} a(\lambda-\mu) y}{\operatorname{sh} a y} \frac{\cos (2 y z+i a(\lambda+\mu))}{\operatorname{sh} \pi y}\right),
$$




$$
D_{a}(z)=\int_{0}^{\infty} \frac{d y}{y^{2}}\left(\frac{a y}{\operatorname{sh} a y} \frac{\sin 2 y z}{2 \operatorname{sh} \pi y}-\frac{z}{\pi}\right)
$$

From straightforward estimates one sees that these representations entail the limits

$$
\begin{gathered}
\lim _{a \downarrow 0} d_{a}(\lambda, \mu ; z)=-i(\lambda-\mu) \int_{0}^{\infty} \frac{d y}{y}\left(\frac{1}{\pi y}-\frac{\cos 2 y z}{\operatorname{sh} \pi y}\right), \\
\lim _{a \downarrow 0} D_{a}(z)=\int_{0}^{\infty} \frac{d y}{y^{2}}\left(\frac{\sin 2 y z}{2 \operatorname{sh} \pi y}-\frac{z}{\pi}\right)
\end{gathered}
$$

and boundedness for $(a, z) \in(0, \pi / 4] \times K$, with $K$ a compact subset of $|\operatorname{Im} z|<\pi / 2$.

Invoking now Vitali's theorem and recalling the identity (3.21), it follows that (3.85) and (3.86) hold true uniformly on compacts in $|\operatorname{Im} z|<\pi / 2$. Next, we exploit Theorem II.4 to obtain uniform convergence on compacts in the right half plane (2.73). To this end we need only observe that the A $\Delta$ Es with step size $a$ obeyed by $\partial_{z}^{2} d_{a}$ and $\partial_{z}^{3} D_{a}$ satisfy all of the assumptions of Theorem II.4, cf. the proof of Prop. III.1. Similarly, we infer uniform convergence on compacts of the left half plane. Since any compact in $\mathrm{C}(\pi)$ can be written as a union of three compacts in the strip $|\operatorname{Im} z|<\pi / 2$ and in the left and right half planes, the proposition now follows.

We point out that (3.85) amounts to

$$
\lim _{a \downarrow 0} \frac{G(\pi, a ; z+i \lambda a)}{G(\pi, a ; z+i \mu a)}=\exp ((\lambda-\mu) \ln (2 \operatorname{ch} z)), \quad \lambda, \mu \in \mathbb{R},
$$

uniformly on compacts in $\mathbb{C}(\pi)$. Observe that the rhs is not meromorphic, unless $\lambda-\mu \in \mathbb{Z}$. The emergence of branch cuts can be understood from the coalescence of zeros and poles taking place for $a \rightarrow 0$, cf. Prop. III.3.

\section{B. The elliptic case}

In this subsection we are concerned with a function that is a minimal solution to three $\mathrm{A} \Delta \mathrm{Es}$ generalizing the hyperbolic $\mathrm{A} \Delta \mathrm{Es}$ (3.4) and (3.5). We study this function along the same lines as in Subsection III A. Our starting point is the infinite series

$$
\sum_{n=1}^{\infty} \frac{\sin 2 n r z}{2 n \operatorname{sh} n r a_{+} \operatorname{sh} n r a_{-}} \equiv g\left(r, a_{+}, a_{-} ; z\right),
$$

where we take at first $r, a_{\delta} \in(0, \infty), \delta=+,-$. Clearly, this series converges absolutely and uniformly for $z$ in an arbitrary compact of the strip $S$ (3.2), so it defines a function $g$ that is analytic in $S$. As before, it is convenient to suppress the dependence on the parameters whenever this causes no confusion. With this convention, our goal is to study the function $G(z)(3.3)$.

To this end we introduce the "right-hand side function"

$$
R(r, a ; z) \equiv-2 i r e^{-a r / 2} \prod_{k=1}^{\infty}\left(1-e^{-2 k a r}\right)^{2} \cdot e^{i r z} s(r, a ; z+i a / 2) .
$$

Using the definition (2.89) of $s$ and the product representation (2.84) of the $\sigma$-function, one easily verifies that $R$ can be rewritten

$$
R(r, a ; z)=\prod_{k=1}^{\infty}(1-\exp (2 i r z-(2 k-1) a r))(z \rightarrow-z),
$$


where the infinite product converges absolutely and uniformly on compacts. From this one readily obtains the representation

$$
R(r, a ; z)=\exp \left(-\sum_{n=1}^{\infty} \frac{\cos 2 n r z}{n \operatorname{sh} n r a}\right), \quad|\operatorname{Im} z|<a / 2 .
$$

(Use the power series for $\ln (1-x)$ to verify this; cf. also (2.93).)

In the sequel it is convenient to employ the abbreviations

$$
\begin{gathered}
q_{\delta} \equiv \exp \left(-a_{\delta} r\right), \\
c_{\delta} \equiv-2 i r q_{\delta}^{1 / 2} \prod_{k=1}^{\infty}\left(1-q_{\delta}^{2 k}\right)^{2}, \\
s_{\delta}(z) \equiv s\left(r, a_{\delta} ; z\right), \\
R_{\delta}(z) \equiv R\left(r, a_{\delta} ; z\right)=c_{\delta} e^{i r z} s_{\delta}\left(z+i a_{\delta} / 2\right),
\end{gathered}
$$

where $\delta=+,-$. We are now prepared for the following proposition.

Proposition III.8 (defining A $\mathbf{A E s ) : ~ W i t h ~ ( 3 . 4 ) ~ r e p l a c e d ~ b y ~}$

$$
\frac{G\left(z+i a_{\delta} / 2\right)}{G\left(z-i a_{\delta} / 2\right)}=R_{-\delta}(z), \quad \delta=+,-,
$$

and (3.5) by

$$
\frac{G\left(z+i\left(a_{+}-a_{-}\right) / 2\right)}{G\left(z-i\left(a_{+}-a_{-}\right) / 2\right)}=\prod_{k=1}^{\infty}\left(\frac{1-q_{-}^{2 k}}{1-q_{+}^{2 k}}\right)^{2} \cdot \frac{s_{-}(z)}{s_{+}(z)},
$$

the assertions of Prop. III.1 hold true.

Proof: In view of (3.99) and (3.95), Theorem II.5 may be invoked to solve the additive form of (3.100). Specifically, we may take

$$
\phi(z) \equiv-\sum_{n \in \mathbb{Z}^{*}} \frac{e^{2 i n r z}}{2 n \operatorname{sh} n r a_{-\delta}},
$$

$s=a_{-} d 2$ and $a=a_{\delta}$. The solution given by (2.106) is then equal to $i g\left(r, a_{+}, a_{-} ; z\right)$ [cf. (3.92)], and so (3.100) follows.

Next, we use (3.20) and the $\mathrm{A} \Delta \mathrm{Es}(3.100)$ to conclude that (3.101) amounts to the identity

$$
\frac{s_{-}(z)}{s_{+}(z)}=\prod_{k=1}^{\infty}\left(\frac{1-q_{+}^{2 k}}{1-q_{-}^{2 k}}\right)^{2} \cdot \frac{R_{-}\left(z-i a_{-} / 2\right)}{R_{+}\left(z-i a_{+} / 2\right)} .
$$

This identity can be deduced from (3.96)-(3.99), so the proposition follows.

Proposition III.9 (automorphy properties): The function $G$ is periodic with primitive period $\pi / r$. It obeys the multiplication formula (3.25) and the period doubling formula

$$
G\left(2 r, a_{+}, a_{-} ; z\right)=G\left(r, a_{+}, a_{-} ; z\right) G\left(r, a_{+}, a_{-} ; z-\pi / 2 r\right) .
$$

Moreover, it satisfies (3.22), (3.23), the scaling relation

$$
G\left(\lambda^{-1} r, \lambda a_{+}, \lambda a_{-} ; \lambda z\right)=G\left(r, a_{+}, a_{-} ; z\right), \quad \lambda \in(0, \infty),
$$


and the duplication formula

$$
\begin{aligned}
G\left(r, a_{+}, a_{-} ; 2 z\right)= & \prod_{l, m=+,-} G\left(r, a_{+}, a_{-} ; z-i\left(l a_{+}+m a_{-}\right) / 4\right) \\
& \times G\left(r, a_{+}, a_{-} ; z-i\left(l a_{+}+m a_{-}\right) / 4-\pi / 2 r\right) .
\end{aligned}
$$

Proof: These features follow from the series representation (3.92) in the same way as in the hyperbolic case. (Combine (3.25), (3.104) and (3.105) to check (3.106).)

Proposition III.10 (zeros, poles, residues): The zeros and poles of $G(z)$ are given by

$$
\begin{gathered}
z_{j k l}^{+} \equiv j \pi / r+z_{k l}^{+}, \quad j \in \mathbb{Z}, \quad k, l \in \mathbb{N} \quad \text { (zeros), } \\
z_{j k l}^{-} \equiv-z_{j k l}^{+}, \quad j \in \mathbb{Z}, \quad k, l \in \mathbb{N} \quad \text { (poles), }
\end{gathered}
$$

with $z_{k l}^{+}$defined by (3.26). The multiplicities of the poles $z_{j k_{0} l_{0}}^{-}$and zeros $z_{j k_{0} l_{0}}^{+}, j \in \mathbb{Z}$, are equal to the number of distinct pairs $(k, l) \in \mathbb{N}^{2}$ such that $z_{k l}^{+}=z_{k_{0} l_{0}}^{+}$. The poles at $z_{j 00}^{-}, j \in \mathbb{Z}$, are simple and have residue

$$
r_{00}=i\left(2 r \prod_{n=1}^{\infty}\left(1-q_{-}^{2 n}\right)\left(1-q_{+}^{2 n}\right)\right)^{-1}
$$

Whenever

$$
e_{k l} \equiv \prod_{m=1}^{k} i s_{-}\left(i m a_{+}\right) \prod_{n=1}^{l} i s_{+}\left(i n a_{-}\right)
$$

is non-zero, the poles at $\overline{z_{k l}}, j \in \mathbb{Z}$, are simple and have residue

$$
r_{k l}=(-)^{k l}\left(\frac{1}{2 r}\right)^{k+l} q_{-}^{\left(l^{2}+l\right)(k+1 / 2)} q_{+}^{\left(k^{2}+k\right)(l+1 / 2)} \prod_{n=1}^{\infty}\left(1-q_{-}^{2 n}\right)^{-2 k}\left(1-q_{+}^{2 n}\right)^{-2 l} \cdot r_{00} / e_{k l} \text {. }
$$

Conversely, if $z_{j k l}^{-}$is a simple pole, then $\boldsymbol{e}_{k l} \neq 0$.

Proof: We proceed along the same lines as in the proof of Prop. III.3. Here, (3.31) holds true with (3.32) replaced by

$$
P_{M}(z) \equiv\left(\prod_{m=1}^{M} R_{-}\left(z-i a_{+}(m-1 / 2)\right)\right)^{-1}
$$

and then the poles of $P_{M}(z)$ are located at $j \pi / r+z_{m l}$, with $j \in \mathbb{Z}$ and $z_{m l}$ given by (3.33). By periodicity we may restrict attention to poles and zeros on the imaginary axis. In view of (3.22) the first two assertions then follow just as in the hyperbolic case.

Turning to the third one, we now get

$$
G\left(z-i\left(a_{+}+a_{-}\right) / 2\right)=\left(c_{-} \exp \left[i r\left(z-i a_{-} / 2\right)\right] s_{-}(z)\right)^{-1} G\left(z+i\left(a_{+}-a_{-}\right) / 2\right)
$$

so that [cf. (3.96) and (3.97)]

$$
r_{00}=\frac{i}{2 r_{n}} \prod_{n=1}^{\infty}\left(1-q_{-}^{2 n}\right)^{-2} G\left(i\left(a_{+}-a_{-}\right) / 2\right) .
$$


Using symmetry in $a_{+}, a_{-}$, we deduce

$$
G\left(i\left(a_{+}-a_{-}\right) / 2\right)=\prod_{n=1}^{\infty} \frac{\left(1-q_{-}^{2 n}\right)}{\left(1-q_{+}^{2 n}\right)}
$$

and so (3.109) follows. (Note that (3.115) can also be derived from (3.101).)

Finally, from the $A \Delta E s$ (3.100) we calculate

$$
\begin{aligned}
G\left(z+z_{k l}^{-}\right)= & (-)^{k l}\left(c_{-}^{k} c_{+}^{l} \exp \left(\frac{r a_{-}}{2}\left[\left(l^{2}+l\right)(2 k+1)+k\right]+\frac{r a_{+}}{2}\left[\left(k^{2}+k\right)(2 l+1)+l\right]\right)\right. \\
& \left.\cdot \exp (\operatorname{irz}[k+l+2 k l]) \prod_{m=1}^{k} s_{-}\left(z-i m a_{+}\right) \prod_{n=1}^{l} s_{+}\left(z-i n a_{-}\right)\right)^{-1} \cdot G\left(z+z_{00}\right) .
\end{aligned}
$$

Using (3.96) and (3.97), the remaining assertions readily follow from this.

At the elliptic level the choice $a_{+}=a_{-}$does not appear to yield extra information, as compared to the general case. But since $G$ is $\pi / r$-periodic, there is no analog of Prop. III.4, and so we do not need additional information on this special case.

Next, we turn to an analog of Prop. III.5.

Proposition III.11 (meromorphic continuation): The function $G$ admits the representation

$$
G\left(r, a_{+}, a_{-} ; z\right)=\prod_{m, n=1}^{\infty} \frac{1-q_{+}^{2 m-1} q_{-}^{2 n-1} e^{-2 i r z}}{1-q_{+}^{2 m-1} q_{-}^{2 n-1} e^{2 i r z}}, \quad q_{\delta} \equiv \exp \left(-a_{\delta} r\right) .
$$

It can be analytically continued to a function that is meromorphic in $r, a_{+}, a_{-}$and $z$, provided $a_{+} r$ and $a_{-} r$ stay in the right half plane. Fixing $r, a_{+}, a_{-}$with $\operatorname{Re}\left(a_{+} r\right)$ and $\operatorname{Re}\left(a_{-} r\right)$ positive, one obtains a meromorphic function whose zeros and poles are located at (3.107) and (3.108), resp.

Proof: It suffices to prove (3.117), since the remaining assertions are clear from this formula. To this end we observe that the numerator infinite product is the downward iteration solution to both of the A $A E s$

$$
\frac{F\left(z+i a_{\delta} / 2\right)}{F\left(z-i a_{\delta} / 2\right)}=R^{(-)}\left(a_{-\delta} ; z\right), \quad \delta=+,-
$$

with

$$
R^{(-)}(a ; z) \equiv \prod_{k=1}^{\infty}\left(1-e^{-(2 k-1) a r} e^{-2 i r z}\right)
$$

Similarly, the denominator infinite product is the upward iteration solution to

$$
\frac{F\left(z+i a_{\delta} / 2\right)}{F\left(z-i a_{\delta} / 2\right)}=R^{(+)}\left(a_{-\delta} ; z\right), \quad \delta=+,-,
$$

with

$$
R^{(+)}(a ; z) \equiv R^{(-)}(a ;-z)
$$

cf. (2.1)-(2.5). But we have 


$$
R^{(+)}\left(a_{\delta} ; z\right) R^{(-)}\left(a_{\delta} ; z\right)=R_{\delta}(z),
$$

cf. (3.94), so the rhs $\widetilde{G}$ of (3.117) solves the $\mathrm{A} \Delta \mathrm{E}$ (3.100). Since both solutions $G$ and $\widetilde{G}$ are $\pi / r$-periodic, have no zeros and poles in the strip $|\operatorname{Im} z| \leqslant a_{\delta} / 2$, and satisfy $G(0)=\widetilde{G}(0)=1$, we deduce $G=\widetilde{G}$.

We continue by detailing the relation of the elliptic $G$-function to the hyperbolic $G$-function. This relation is the first instance of a general type of limiting transition between meromorphic functions that will reappear several times. Therefore, it is convenient to introduce a term referring to the type of limit involved.

To this end, assume $f_{p}(z)$ is a family of meromorphic functions parametrized by $p \in C^{N}$. We shall say that $f_{p}(z)$ converges mero-uniformly to a meromorphic function $f(z)$ as $p \rightarrow p_{0}$ whenever one has $f_{p}(z) \rightarrow f(z)$ uniformly on compacts not containing poles of $f(z)$, and $1 / f_{p}(z) \rightarrow 1 / f(z)$ uniformly on compacts not containing zeros of $f(z)$. (Equivalently, viewing meromorphic functions as holomorphic functions from $\mathrm{C}$ to the Riemann sphere $\mathrm{P}^{1}$, one has $f_{p} \rightarrow f$ mero-uniformly as $p \rightarrow p_{0}$ iff the convergence is $\mathrm{P}^{\mathrm{l}}$-uniform on arbitrary C-compacts.)

Defining the renormalized function

$$
G_{\mathrm{ren}}\left(r, a_{+}, a_{-} ; z\right)=G\left(r, a_{+}, a_{-} ; z\right) \exp \left(\frac{\pi^{2} z}{6 i r a_{+} a_{-}}\right)
$$

we are now prepared for the next proposition.

Proposition III.12 (relation to hyperbolic $G$-function): Fixing $a_{+}, a_{-}>0$, one has

$$
\lim _{r \downarrow 0} G_{\mathrm{ren}}\left(r, a_{+}, a_{-} ; z\right)=G\left(a_{+}, a_{-} ; z\right),
$$

where the limit is mero-uniform.

Proof: Writing $G_{\text {ren }}=\exp \left(i g_{\text {ren }}\right)$, we obtain

$$
g_{\text {ren }}\left(r, a_{+}, a_{-} ; z\right)=r \sum_{n=1}^{\infty} \frac{1}{n r}\left(\frac{\sin 2 n r z}{2 \operatorname{sh} n r a_{+} \operatorname{sh} n r a_{-}}-\frac{z}{n r a_{+} a_{-}}\right), \quad z \in S ;
$$

cf. (3.92). Comparing to (3.1), a routine dominated convergence argument now yields

$$
\lim _{r \downarrow 0} g_{\mathrm{ren}}\left(r, a_{+}, a_{-} ; z\right)=g\left(a_{+}, a_{-} ; z\right), \quad z \in S,
$$

uniformly on $S$-compacts.

Next, we note that $G_{\text {ren }}$ satisfies the $\mathrm{A} \Delta \mathrm{E}$

$$
\frac{G\left(z+i a_{+} / 2\right)}{G\left(z-i a_{+} / 2\right)}=R_{-, \text {ren }}(z)
$$

with

$$
R_{-, \text {ren }}(z) \equiv \exp \left(\frac{\pi^{2}}{6 r a_{-}}\right) R_{-}(z)
$$

In view of (3.126) this entails that for $|\operatorname{Im} z| \leqslant a_{-} / 2$ we have

$$
\lim _{r \downarrow 0} R_{-, \operatorname{ren}}(z)=\frac{G\left(a_{+}, a_{-} ; z+i a_{+} / 2\right)}{G\left(a_{+}, a_{-} ; z-i a_{+} / 2\right)}=2 \operatorname{ch} \frac{\pi z}{a_{-}},
$$


where we used (3.4). Recalling (3.99) and the limit (2.92), we deduce

$$
\lim _{r \downarrow 0} \exp \left(\frac{\pi^{2}}{6 r a_{-}}\right)\left(i c_{-}\right)=\frac{2 \pi}{a_{-}} .
$$

Using then (2.92) once more, one sees that (3.129) holds uniformly on C-compacts. Therefore, one may exploit the $\mathrm{A} \Delta \mathrm{E}$ (3.127) and uniform convergence of $G_{\text {ren }}$ to $G$ on $S$-compacts to obtain uniform convergence on C-compacts that do not contain the poles $z_{j k}^{-}, j, k \in \mathbb{N}$, of $G$. Moreover, (3.126) entails uniform convergence of $1 / G_{\text {ren }}$ to $1 / G$ on $S$-compacts, so one can also use (3.127) and (3.129) to infer $1 / G_{\mathrm{ren}} \rightarrow 1 / G$ uniformly on compacts not containing the zeros $z_{j k}^{+}$.

As a corollary of the proof we obtain the limit

$$
\lim _{r \downarrow 0} r \exp \left(\frac{\pi^{2}}{6 r a}\right) \prod_{n=1}^{\infty}\left(1-e^{-2 n a r}\right)^{2}=\frac{\pi}{a}, \quad a>0 ;
$$

cf. (3.130) and (3.97). Equivalently, this can be written

$$
\lim _{r \downarrow 0}\left(\sum_{n=1}^{\infty} \frac{1}{n}\left(\frac{e^{-n r a}}{\operatorname{shn} r a}-\frac{1}{n r a}\right)-\ln r\right)=\ln \frac{a}{\pi}, \quad a>0 .
$$

The last proposition of this subsection is the analog of Prop. III.7 in the previous one. To state it, we introduce the cut plane

$$
\mathrm{C}(r, d) \equiv \mathrm{C}\{ \pm i[d / 2, \infty)+k \pi / r \mid k \in \mathbb{Z}\}, \quad r, d>0,
$$

and define a branch $g(r, A, a ; z)$ of $-i \ln G$ in $C(r, A+a)$ via (3.93) for $|\operatorname{Im} z|<(A+a) / 2$. Then we set

$$
\begin{gathered}
d_{a}(r, A, \lambda, \mu ; z) \equiv g(r, A, a ; z+i \lambda a)-g(r, A, a ; z+i \mu a), \quad z \in \mathbb{C}(r, A+a), \quad \lambda, \mu \in \mathbb{R}, \\
D_{a}(r, A ; z) \equiv a g(r, A, a ; z), \quad z \in \mathbb{C}(r, A+a)
\end{gathered}
$$

(This should be compared to (3.83) and (3.84).)

Proposition III.13 (zero step size limits): One has

$$
\begin{gathered}
\lim _{a \downarrow 0} d_{a}(r, A, \lambda, \mu ; z)=-i(\lambda-\mu) \ln R(r, A ; z), \quad \lambda, \mu \in \mathbb{R}, \\
\lim _{a \downarrow 0} D_{a}(r, A ; z)=-\int_{0}^{z} d w \ln R(r, A ; w),
\end{gathered}
$$

uniformly on compact subsets of the cut plane $\mathrm{C}(r, A)$ (3.133). Here, $\ln$ is real-valued for $z$ and $w$ real, resp., and the integration path in (3.137) belongs to $\mathbb{C}(r, A)$.

Proof: This follows in the same way as Prop. III.7, with (3.93), (the logarithm of) (3.95) and Theorem II.7 playing the role of (3.1), (3.21) and Theorem II.4, resp. (Since the limits are $\pi / r$-periodic in the strip $|\operatorname{Im} z|<A / 2$, one need only handle compacts in $\mathscr{B}_{r}(2.113)$.)

In terms of $G,(3.136)$ reads

$$
\lim _{a \downarrow 0} \frac{G(r, A, a ; z+i \lambda a)}{G(r, A, a ; z+i \mu a)}=\exp ((\lambda-\mu) \ln R(r, A ; z)), \quad \lambda, \mu \in \mathbb{R},
$$


uniformly on compacts in $\mathbb{C}(r, A)$. Once more, the branch cuts arise from coalescence of zeros and poles, cf. Prop. III.10.

\section{The trigonometric case}

The trigonometric case is most easily understood by viewing it as a limiting case of the elliptic case. In view of (2.90), this should involve sending one of $a_{+}, a_{-}$to $\infty$. We shall fix $a_{+} \equiv a$ $\in(0, \infty)$ and let $a_{-} \equiv A$ go to $\infty$. To get finite limits, we clearly should shift $z$ in an $A$-dependent way. We take $z \rightarrow z-i A / 2$, and thus wind up with

$$
G(r, a ; z) \equiv \lim _{A \rightarrow \infty} G(r, a, A ; z-i A / 2)
$$

From the product representation (3.117) it is immediate that this limit exists mero-uniformly, yielding

$$
G(r, a ; z)=\prod_{m=1}^{\infty}\left(1-q^{2 m-1} e^{2 i r z}\right)^{-1}, \quad q \equiv e^{-a r} .
$$

For $\operatorname{Im} z>-a / 2$ we can also evaluate the limit (3.139) by using (3.92); this yields the series representation

$$
G(r, a ; z)=\exp \left(\sum_{n=1}^{\infty} \frac{e^{2 i n r z}}{2 n \operatorname{sh} n r a}\right), \quad \operatorname{Im} z>-a / 2 .
$$

We continue by studying the trigonometric $G$-function just defined.

Proposition III.14 (defining $\mathbf{A} \mathbf{\Delta E}$ ): The function $G(r, a ; z)$ is the upward iteration solution to the $A \Delta E$

$$
\frac{G(z+i a / 2)}{G(z-i a / 2)}=1-e^{2 i r z}
$$

Proof: This is clear from the product representation (3.140) [recall (2.1)-(2.4)].

Notice that the $\mathrm{A} \Delta \mathrm{E}$ (3.142) is not regular. However, a shift $z \rightarrow z+i a / 2$ (say) gives rise to a regular $\mathrm{A} \Delta \mathrm{E}$. Indeed, the function

$$
\phi(z)=\ln (1-\exp (2 i r(z+i a / 2)))=-\sum_{n=1}^{\infty} n^{-1} q^{n} e^{2 i n r z}
$$

satisfies the assumptions of Theorem II.4, and $G(r, a ; z+i a / 2)$ is a minimal solution to the associated multiplicative $\mathrm{A} \Delta \mathrm{E}$. [Compare the logarithm of (3.141) with (2.106) to see this.] Observe also that (3.142) agrees with the $A \rightarrow \infty$ limit of the elliptic $\mathrm{A} \Delta \mathrm{E}$

$$
\frac{G(r, a, A ; z-i A / 2+i a / 2)}{G(r, a, A ; z-i A / 2-i a / 2)}=-2 i r \prod_{n=1}^{\infty}\left(1-e^{-2 n A r}\right)^{2} \cdot e^{i r z} s(r, A ; z),
$$

cf. (3.100), (3.96)-(3.99), (3.139) and (2.90).

Proposition III.15 (automorphy properties): The function $G$ is periodic with primitive period $\pi / r$. It obeys the multiplication formula

$$
G\left(r, \frac{a}{M} ; z\right)=\prod_{j=1}^{M} G\left(r, a ; z+\frac{i a}{2 M}(M+1-2 j)\right),
$$


the period doubling formula

$$
G(2 r, a ; z)=G(r, a ; z) G(r, a ; z-\pi / 2 r)
$$

the scaling relation

$$
G\left(\lambda^{-1} r, \lambda a ; \lambda z\right)=G(r, a ; z), \quad \lambda \in(0, \infty),
$$

and the duplication formula

$$
G(r, a ; 2 z)=\prod_{\sigma=+,-} G(r, a ; z-i \sigma a / 4) G(r, a ; z-i \sigma a / 4-\pi / 2 r)
$$

Proof: These properties follow from the series representation (3.141) in the same way as in the two previous cases.

Proposition III.16 (zeros, poles, residues): The function $G(z)$ has no zeros and simple poles given by

$$
z_{j k} \equiv j \pi / r-i a(k+1 / 2), \quad j \in \mathbb{Z}, \quad k \in \mathbb{N} \quad \text { (poles). }
$$

The residues at the poles $z_{j 0}, j \in \mathbb{Z}$, are given by

$$
r_{0}=i\left(2 r \prod_{n=1}^{\infty}\left(1-q^{2 n}\right)\right)^{-1}=\frac{i}{2 r} G(i a / 2),
$$

and the residues at the remaining poles $z_{j k}, j \in \mathbb{Z}, k \in \mathbb{N}^{*}$, are given by

$$
r_{k}=r_{0} / \prod_{m=1}^{k}\left(1-q^{-2 m}\right)
$$

Proof: The first assertion is immediate from (3.140). The residues (3.150) follow either from (3.109) by taking a limit, or directly from (3.140). Using

$$
G\left(z+z_{0 k}\right)=\prod_{m=1}^{k}\left(1-q^{-2 m} e^{2 i r z}\right)^{-1} G\left(z+z_{00}\right),
$$

the residues at the remaining poles can now be obtained, yielding (3.151).

Proposition III.17 (asymptotics): The function $G$ satisfies the bound

$$
G(r, a ; z)=1+O(\exp (-2 r \operatorname{Im} z)), \quad \operatorname{Im} z \rightarrow \infty,
$$

uniformly for $\operatorname{Rez} \in \mathbb{R}$.

Proof: This estimate readily follows from the series representation (3.141).

Proposition III.18 (meromorphic continuation): The function $G$ can be analytically continued to a function that is meromorphic in $r, a$ and $z$, provided ar stays in the right half plane. Fixing $r, a$ with $\operatorname{Re}(a r)>0$, one obtains a meromorphic function without zeros and with simple poles located at (3.149).

Proof: This can be read off from the product representation (3.140).

The propositions derived thus far have elliptic and/or hyperbolic analogs. In the previous two cases, however, the $G$-function satisfies $G(z) G(-z)=1$, a relation that does not hold in the trigonometric case. Instead, we have the following result.

Proposition III.19 (functional equation): The trigonometric G-function satisfies 


$$
G(r, a ; z) G(r, a ;-z)=R(r, a ; z)^{-1},
$$

where the rhs is given by (3.93).

Proof: This is obvious from the series representations (3.141) and (3.95).

We point out that this functional equation may be seen as a footprint left by the second $\mathrm{A} \Delta \mathrm{E}$ satisfied by the elliptic $G$-function: Taking $a \rightarrow a_{+}$, the rhs can be written $R_{+}(z)^{-1}$, so (3.154) can be deduced from (3.100) with $\delta=-$ and the limit (3.139).

Next, we introduce the function

$$
T(r ; z) \equiv \frac{G(r, 1 ; 0)}{G(r, 1 ;-z)} \exp \left(\frac{r z^{2}}{2}+i z \ln (2 r)-\frac{1}{2} \ln \pi\right), \quad \operatorname{Re} r>0 .
$$

This renormalized version of $G(r, a ; z)$ satisfies the $\mathrm{A} \Delta \mathrm{E}$

$$
\frac{T(r ; z+i / 2)}{T(r ; z-i / 2)}=\frac{i \sin r z}{r}
$$

and functional equation

$$
T(r ; z) T(r ;-z)=\pi^{-1} \exp \left(r z^{2}+i r z\right) \frac{s(r, 1 ; z+i / 2)}{s(r, 1 ; i / 2)} .
$$

Taking $r \downarrow 0$, the right-hand sides of (3.156) and (3.157) obviously converge to $i z$ and $\pi^{-1} \operatorname{ch} \pi z$ [recall (2.92)], resp., in accordance with the next proposition.

Proposition III.20 (relation to gamma function): One has

$$
\lim _{r \downarrow 0} T(r ; z)=1 / \Gamma(i z+1 / 2)
$$

uniformly for $z$ in C-compacts.

Proof: We begin by noting that it suffices to show that (3.158) holds uniformly on compacts of the lower half plane (LHP). (Indeed, from (3.156) we have

$$
T(r ; z+i k)=\frac{i}{r} \sin r(z+i(k-1 / 2)) \cdots \frac{i}{r} \sin r(z+i / 2) T(r ; z),
$$

so if (3.158) holds uniformly on LHP-compacts, then the rhs of (3.159) converges in the same sense to

$$
(i z-k+1 / 2) \cdots(i z-1 / 2) \frac{1}{\Gamma(i z+1 / 2)}=\frac{1}{\Gamma(i(z+i k)+1 / 2)} .
$$

Hence, (3.158) follows for compacts of $\operatorname{Im} z \leqslant k$ ). To this end we use the formula

$$
e(z)=e(0)+z e^{\prime}(0)+\int_{0}^{z} d w \int_{0}^{w} d s e^{\prime \prime}(s)
$$

to rewrite the logarithms of $T(r ; z)$ and $1 / \Gamma(i z+1 / 2)$. This yields

$$
T(r ; z)=\exp \left(-\frac{1}{2} \ln \pi+i z K(r)+\int_{0}^{z} d w \int_{0}^{w} d \operatorname{sh}(r ; s)\right)
$$

with 


$$
\begin{gathered}
h(r ; z) \equiv 2 r \sum_{n=1}^{\infty} \frac{n r e^{-2 i n r z}}{\operatorname{sh} n r}+r, \quad \operatorname{Im} z<1 / 2, \\
K(r) \equiv \ln (2 r)+\sum_{n=1}^{\infty} \frac{r}{\operatorname{sh} n r}
\end{gathered}
$$

[cf. (3.155) and (3.141)] and

$$
\frac{1}{\Gamma(i z+1 / 2)}=\exp \left(-\frac{1}{2} \ln \pi-i z \psi\left(\frac{1}{2}\right)+\int_{0}^{z} d w \int_{0}^{w} d \operatorname{sh}(s)\right)
$$

with

$$
h(z) \equiv 2 \int_{0}^{\infty} d y \frac{y e^{-2 i y z}}{\operatorname{sh} y}, \quad \operatorname{Im} z<1 / 2,
$$

cf. (A37), (A12), and (A33), (A34).

Comparing (3.163) and (3.166), we deduce

$$
\lim _{r \downarrow 0} h(r ; z)=h(z)
$$

uniformly on LHP-compacts. Comparing then (3.162) with (3.165), we see that it remains to show

$$
\lim _{r \downarrow 0} K(r)=-\psi\left(\frac{1}{2}\right) .
$$

To prove this, we use the $\mathrm{A} \Delta \mathrm{Es}$ (3.156) and (A24) to write

$$
\frac{T(r ;-i)}{T(r ; 0)} \frac{\Gamma(3 / 2)}{\Gamma(1 / 2)}=\frac{r}{2 \operatorname{sh}(r / 2)} .
$$

Due to (3.162) and (3.165), the lhs can be rewritten

$$
\exp \left(K(r)+\psi\left(\frac{1}{2}\right)+\int_{0}^{-i} d w \int_{0}^{w} d s[h(r ; s)-h(s)]\right)
$$

and since the integral converges to 0 for $r \downarrow 0$ we now obtain (3.168). Therefore, the proof of the proposition is complete.

Comparing the A $\Delta \mathrm{Es}$ (3.156) and (3.70), we deduce that the quotient

$$
Q(r ; z) \equiv T(r ; z) / H(i r / \pi ; z), \quad \operatorname{Re} r>0
$$

of the trigonometric and hyperbolic functions is $i$-periodic. Moreover, comparing poles and zeros of $T$ and $H$, we deduce that $Q$ is entire in $z$ and has simple zeros at

$$
z=-k \pi / r+i(l+1 / 2), \quad k \in \mathbb{N}^{*}, \quad l \in \mathbb{Z} .
$$

Furthermore, recalling Prop. III.6, we infer

$$
\lim _{r \downarrow 0} Q(r ; z)=1 \text { (uniformly on compacts). }
$$


Our last proposition concerns two zero step size limits that may be tied in with (3.136) and (3.137) via (3.139). We set

$$
\mathrm{C}_{-}(r, d) \equiv \mathbb{C}\{-i[d / 2, \infty)+k \pi / r \mid k \in \mathbb{Z}\}, \quad r>0, d \geqslant 0,
$$

and define a branch $g(r, a ; z)$ of $-i \ln G$ in $\mathrm{C}_{-}(r, a)$ by requiring

$$
g(r, a ; z) \equiv-i \sum_{n=1}^{\infty} \frac{e^{2 i n r z}}{2 n \operatorname{sh} n r a}, \quad \operatorname{Im} z>-a / 2
$$

cf. (3.141). Now we put

$$
\begin{gathered}
d_{a}(r, \lambda, \mu ; z) \equiv g(r, a ; z+i \lambda a)-g(r, a ; z+i \mu a), \quad z \in \mathbb{C}_{-}(r, a), \quad \lambda, \mu \in \mathbb{R}, \\
D_{a}(r ; z) \equiv a g(r, a ; z), \quad z \in \mathbb{C}_{-}(r, a) .
\end{gathered}
$$

(Compare this to (3.133)-(3.135).)

Proposition III.21 (zero step size limits): One has

$$
\begin{gathered}
\lim _{a \downarrow 0} d_{a}(r, \lambda, \mu ; z)=-i(\lambda-\mu) \ln \left(1-e^{2 i r z}\right), \quad \lambda, \mu \in \mathbb{R}, \\
\lim _{a \downarrow 0} D_{a}(r ; z)=-\int_{i \infty}^{z} d w \ln \left(1-e^{2 i r w}\right),
\end{gathered}
$$

uniformly on compact subsets of the cut plane $\mathrm{C}_{-}(r, 0)$ (3.174). Here, in is real valued for $z, w \in i(0, \infty)$, and the integration path in (3.179) belongs to $\mathbb{C}_{-}(r, 0)$.

Proof: From (3.175) it readily follows that the proposition is valid when the cut plane $\mathrm{C}_{-}(r, 0)$ is replaced by its upper half plane subset. Applying Theorem II.7 to the functions $f_{a}(z) \equiv d_{a}(z+i)$ and $f_{a}(z) \equiv D_{a}(z+i)$ (which satisfy the hypotheses of that theorem for $a_{0}$ small enough), one obtains validity for all of the cut plane.

Translated to $G$, the limit (3.178) becomes

$$
\lim _{a \downarrow 0} \frac{G(r, a ; z+i \lambda a)}{G(r, a ; z+i \mu a)}=\exp \left((\lambda-\mu) \ln \left(1-e^{2 i r z}\right)\right), \quad \lambda, \mu \in \mathbb{R}
$$

uniformly on compact subsets of the cut plane $\mathbb{C}_{-}(r, 0)$. Just as in the previous two cases [cf. (3.91) and (3.138)], this formula is evident from the defining $\mathrm{A} \Delta \mathrm{E}$ when $\lambda-\mu$ is an integer. For $\lambda-\mu \notin \mathbb{Z}$, the branch cuts in the lower half plane arise from the coalescence of poles and zeros that can be read off from (3.149).

We conclude this subsection by detailing the relation of the trigonometric $G$-function $G(r, a ; z)$ to the $\tilde{q}$-gamma function $\Gamma_{\tilde{q}}(z)$. Recall the latter is given by (cf., e.g., Ref. 4, p. 16)

$$
\Gamma_{\widetilde{q}}(z)=(1-\widetilde{q})^{1-z} \prod_{n=1}^{\infty} \frac{\left(1-\widetilde{q}^{n}\right)}{\left(1-\widetilde{q}^{z+n-1}\right)} .
$$

Comparing this to the product formula (3.140) for $G$, we see that when we take

$$
\tilde{q} \equiv q^{2}=e^{-2 a r}
$$

we may write $G$ as 


$$
G(r, a ; a z)=\Gamma_{\bar{q}}(-i z+1 / 2)(1-\widetilde{q})^{-i z-1 / 2} \prod_{n=1}^{\infty}\left(1-\widetilde{q}^{n}\right)^{-1} .
$$

From this we readily obtain [recall (3.155)]

$$
T(r ; z)=\frac{\Gamma_{\vec{q}}(1 / 2)}{\Gamma_{\tilde{q}}(i z+1 / 2)} \exp \left(-\frac{1}{2} \ln \pi+\frac{r z^{2}}{2}-i z \ln \left(\frac{1-e^{-2 r}}{2 r}\right)\right) .
$$

Using these relations, some of the above results can be translated in terms of $\Gamma_{\bar{q}}$, recovering results that have been obtained by several authors, cf. Ref. 4 and references given there.

\section{SCATTERING FUNCTIONS}

\section{A. The hyperbolic case}

We present our results on the hyperbolic scattering function $u\left(a_{+}, a_{-}, b ; z\right)$ in a form that anticipates our account of the elliptic case. First of all, we define $u$ by

$$
u(z) \equiv \frac{G\left(z-i b+i\left(a_{+}+a_{-}\right) / 2\right) G\left(z+i b-i\left(a_{+}+a_{-}\right) / 2\right)}{G\left(z-i\left(a_{+}-a_{-}\right) / 2\right) G\left(z+i\left(a_{+}-a_{-}\right) / 2\right)},
$$

where $G(z)=G\left(a_{+}, a_{-} ; z\right)$ is the hyperbolic $G$-function from Subsection III A. In (4.1) and in many later formulas, the dependence on $a_{+}$and $a_{-}$is suppressed. This should cause no confusion, since $u$-just like $G$-satisfies

$$
u\left(a_{+}, a_{-} ; z\right)=u\left(a_{-}, a_{+} ; z\right),
$$

cf. (3.23). Similarly, the automorphy properties (3.22) and (3.24) yield

$$
\begin{gathered}
u(-z)=1 / u(z), \\
u\left(\lambda a_{+}, \lambda a_{-}, \lambda b ; \lambda z\right)=u\left(a_{+}, a_{-}, b ; z\right), \quad \lambda \in(0, \infty) .
\end{gathered}
$$

By virtue of Prop. III.5 the $u$-function is meromorphic in $a_{+}, a_{-}, b$ and $z$, provided the quotient $a_{+} / a_{-}$stays away from the negative real axis. As a rule, however, we restrict our considerations to parameters in the set

$$
\mathscr{H} \equiv\left\{\left(a_{+}, a_{-}, b\right) \mid a_{+}, a_{-}>0, b \in \mathbb{R}\right\} .
$$

This choice corresponds to physical applications; in particular, it guarantees $|u(x)|=1$ for real $x$.

Next, we observe that the A $\Delta \mathrm{Es}$ (3.4) entail that $u$ solves the ADEs

$$
\frac{u\left(z+i a_{\delta} / 2\right)}{u\left(z-i a_{\delta} / 2\right)}=\frac{s_{-\delta}\left(z-i b+i a_{\delta} / 2\right) s_{-\delta}\left(z+i b-i a_{\delta} / 2\right)}{s_{-\delta}\left(z+i a_{\delta} / 2\right) s_{-\delta}\left(z-i a_{\delta} / 2\right)},
$$

where we have introduced

$$
s_{\delta}(z) \equiv \frac{\operatorname{sh}\left(\pi z / a_{\delta}\right)}{\pi / a_{\delta}}, \quad \delta=+,-.
$$

(This definition mimicks the elliptic definition (3.98), cf. (2.92).) Fixing $\delta \in\{+,-\}$, the $\mathrm{A} \Delta \mathrm{E}$ (4.6) is regular unless the parameters $\left(a_{+}, a_{-}, b\right)$ belong to the planes 


$$
a_{\delta}=2 n a_{-\delta}, \quad n \in \mathbb{N}^{*},
$$

or

$$
b=k a_{-\delta}+a_{\delta} / 2, \quad k \in \mathbb{Z} .
$$

These planes separate the region $\mathscr{H}(4.5)$ into infinitely many connected components, one of which reads

$$
\mathscr{R}_{\delta} \equiv\left\{\left(a_{+}, a_{-}, b\right) \in \mathscr{H} \mid a_{\delta} \in\left(0,2 a_{-\delta}\right), b \in\left(a_{\delta} / 2, a_{-\delta}+a_{\delta} / 2\right) .\right.
$$

Choosing parameters in $\mathscr{B}_{\delta}$, the $u$-function may now be characterized as the unique minimal solution to the $\mathrm{A} \Delta \mathrm{E}(4.6)$ that satisfies

$$
u(0)=1, \quad|u(x)|=1, \quad x \in \mathbb{R} .
$$

Indeed, the pole/zero properties of the $G$-function (cf. Prop. III.3) entail that $u$ (4.1) is a regular solution to (4.6) if and only if $\left(a_{+}, a_{-}, b\right) \in \mathscr{R}_{\delta}$. Moreover, for all $\left(a_{+}, a_{-}, b\right) \in \mathscr{H}$ one has

$$
u(z)=\exp \left( \pm \frac{i \pi}{a_{+} a_{-}}\left(b-a_{+}\right)\left(b-a_{-}\right)\right)+O\left(\exp \left( \pm\left(\epsilon-2 \pi / a_{m}\right) z\right)\right), \quad \operatorname{Re} z \rightarrow \pm \infty,
$$

uniformly for $\operatorname{Im} z$ in $\mathbb{R}$-compacts, cf. Prop. III.4. Therefore, $u$ is indeed a minimal solution to (4.6) for parameters in $\mathscr{R}_{\delta}$ (4.10). From Theorem II.1 and (4.11) one now easily deduces the above uniqueness assertion.

It should be remarked at this point that the $\mathrm{A} \Delta \mathrm{E}$ (4.6) does admit minimal solutions whenever the parameters do not belong to the planes (4.8) and (4.9). Indeed, this readily follows from Section II. More concretely, a minimal solution can be constructed by multiplying $u(z)$ by finitely many factors of the form $s_{\delta}(z-p) / s_{\delta}(z+p)$ that cancel the poles and zeros of $u(z)$ in the strip $|\operatorname{Im} z|<a_{\delta} / 2$. (Observe that $u(z)$ has no poles and zeros for $|\operatorname{Im} z|=a_{\delta} / 2$ unless (4.8) or (4.9) holds true.)

Since the rhs of (4.6) is $a_{-\delta}$-periodic in $b$, the quotient $u\left(b+a_{-\delta} ; z\right) / u(b ; z)$ is $i a_{\delta}$-periodic in $z$. Specifically, one obtains from (4.1) and (3.4)

$$
\frac{u\left(b+a_{-\delta} ; z\right)}{u(b ; z)}=-\frac{s_{\delta}(z+i b)}{s_{\delta}(z-i b)} .
$$

Therefore, iteration yields (taking $k_{+}, k_{-} \in \mathbb{Z}$ )

$$
\frac{u\left(b+k_{+} a_{+}+k_{-} a_{-} ; z\right)}{u(b ; z)}=\prod_{\delta=+,-} \prod_{j_{\delta}=1}^{\left|k_{\delta}\right|} \frac{s_{-\delta}\left(z+i\left(k_{\delta} /\left|k_{\delta}\right|\right)\left(b-a_{\delta} / 2\right)+i a_{\delta}\left(j_{\delta}-1 / 2\right)\right)}{(z \rightarrow-z)}
$$

Next, we introduce the parameter subset

$$
\mathscr{D} \equiv\left\{\left(a_{+}, a_{-}, b\right) \in \mathscr{H} \mid b=k_{+} a_{+}+k_{-} a_{-}, k_{+}, k_{-} \in \mathbb{Z}\right\}
$$

of $\mathscr{H}$ (4.5). Since the numbers $k_{+} a_{+}+k_{-} a_{-}, k_{+}, k_{-} \in \mathbb{Z}$, are dense in $\mathbb{R}$ whenever $a_{+} / a_{-} \notin \mathbb{Q}$, the subset $\mathscr{D}$ is dense in $\mathscr{H}$. Now from (4.1) we read off

$$
u\left(a_{+}, a_{-}, a_{+} ; z\right)=u\left(a_{+}, a_{-}, a_{-} ; z\right)=1
$$

and also, using (3.4), 


$$
u\left(a_{+}, a_{-}, 0 ; z\right)=-1
$$

Hence, (4.14) yields

$$
u\left(a_{+}, a_{-}, k_{+} a_{+}+k_{-} a_{-} ; z\right)=c_{k_{+}, k_{-}} \prod_{\delta=+,-} \prod_{j_{\delta}=1}^{\left|k_{\delta}\right|} \frac{s_{-\delta}\left(z+i a_{\delta}\left(j_{\delta}-\theta\left(k_{\delta}\right)\right)\right)}{(i \rightarrow-i)}
$$

with

$$
\theta(j) \equiv \begin{cases}0, & j<0 \\ 1, & j>0\end{cases}
$$

and

$$
c_{k, l} \equiv(-)^{k+l+1}, \quad k, l \in \mathbb{Z}
$$

In words, the $u$-function is an elementary function for parameters in the dense subset $\mathscr{D}$ of $\mathscr{H}$. (Of course, whenever $a_{-} / a_{+}$is a rational number, there exist infinitely many distinct pairs $(k, l)$ $\in \mathbb{Z}^{2}$ for which the number $k a_{+}+l a_{-}$is the same; this yields different representations for the same function.)

We continue by noting the symmetry property

$$
u(b ; z)=u\left(a_{+}+a_{-}-b ; z\right),
$$

which can be read off from (4.1). Combining this with (4.14) (taking $k_{+}, k_{-}=1$ ), we deduce

$$
\frac{u(-b ; z)}{u(b ; z)}=\frac{s_{+}(z+i b)}{s_{+}(z-i b)} \frac{s_{-}(z+i b)}{s_{-}(z-i b)} .
$$

Since this parameter transformation leaves $\mathscr{D}(4.15)$ invariant, it does not give rise to additional elementary representations for $u$.

Next, we derive analogs of the multiplication formula (3.25). First, we use (4.1) to get

$$
\begin{aligned}
u\left(\frac{a_{+}}{M}, a_{-}, b ; z\right)= & \prod_{j=1}^{M} \frac{G\left(z-i b+i\left(a_{+} / 2\right)+i\left(a_{-} / 2\right)+i\left(a_{+} / M\right)(1-j)\right)}{G\left(z-i\left(a_{+} / 2\right)+i\left(a_{-} / 2\right)+i\left(a_{+} / M\right)(M-j)\right)} \\
& \times \frac{G\left(z+i b-i\left(a_{+} / 2\right)-i\left(a_{-} / 2\right)+i\left(a_{+} / M\right)(M-j)\right)}{G\left(z+i\left(a_{+} / 2\right)-i\left(a_{-} / 2\right)+i\left(a_{+} / M\right)(1-j)\right)} .
\end{aligned}
$$

with $G(z)=G\left(a_{+}, a_{-} ; z\right)$. Rearranging and using (4.1) once more, we deduce

$$
\begin{aligned}
u\left(\frac{a_{+}}{M}, a_{-}, b ; z\right)= & u\left(a_{+}, a_{-}, b ; z\right) \prod_{k=1}^{M-1} u\left(a_{+}, a_{-}, b ; z+i k \frac{a_{+}}{M}\right) \\
& \times \frac{G\left(z+i k\left(a_{+} / M\right)-i b+i\left(a_{-} / 2\right)-i\left(a_{+} / 2\right)\right)}{G\left(z+i k\left(a_{+} / M\right)-i b+i\left(a_{-} / 2\right)+i\left(a_{+} / 2\right)\right)} \\
& \times \frac{G\left(z+i k\left(a_{+} / M\right)-i\left(a_{-} / 2\right)+i\left(a_{+} / 2\right)\right)}{G\left(z+i k\left(a_{+} / M\right)-i\left(a_{-} / 2\right)-i\left(a_{+} / 2\right)\right)} .
\end{aligned}
$$

This can be simplified by using the $\mathrm{A} \Delta \mathrm{E}$ (3.4), which yields 


$$
u\left(\frac{a_{+}}{M}, a_{-}, b ; z\right)=(-)^{M-1} \prod_{k=0}^{M-1} u\left(a_{+}, a_{-}, b ; z+i k \frac{a_{+}}{M}\right) \prod_{j=1}^{M-1} \frac{s_{-}\left(z+i j a_{+} / M\right)}{s_{-}\left(z-i b+i j a_{+} / M\right)}
$$

Equivalently, we may also rearrange (4.23) to get

$$
u\left(\frac{a_{+}}{M}, a_{-}, b ; z\right)=(-)^{M-1} \prod_{k=0}^{M-1} u\left(a_{+}, a_{-}, b ; z-i k \frac{a_{+}}{M}\right) \prod_{j=1}^{M-1} \frac{s_{-}\left(z+i b-i j a_{+} / M\right)}{s_{-}\left(z-i j a_{+} / M\right)}
$$

Substituting $a_{-} \rightarrow a_{-} / N$ in the formulas (4.25) and (4.26), and using first (4.2) and then one of these formulas again, one obtains four representations for $u\left(a_{+} / M, a_{-} / N, b ; z\right)$ in terms of $u\left(a_{+}, a_{-}, b ; z\right)$ and sh-quotients.

The choices $b=a_{+} / 2$ or $b=a_{-} / 2$ yield the sine-Gordon soliton-soliton $S$-matrix. Taking $b=a_{+} / 2$, it follows from (4.18) that there exists a dense set of $a_{-}$-choices yielding an elementary $u$. Specifically, choosing $a_{-}=a_{+}(1+2 j) / 2 l$ with $j \in \mathbb{N}, l \in \mathbb{N}^{*}$, we have $b=a_{+} / 2=l a_{-}-j a_{+}$. Thus, setting

$$
\alpha_{j l} \equiv \frac{\pi}{2 l}(1+2 j), \quad j \in \mathbb{N}, \quad l \in \mathbb{N}^{*},
$$

we deduce from (4.18)

$$
u\left(\pi, \alpha_{j l}, \pi / 2 ; z\right)=\prod_{m=1}^{j} \frac{\operatorname{sh} \pi \alpha_{j l}^{-1}(z+i m \pi)}{(z \rightarrow-z)} \prod_{k=1}^{l-1} \frac{\operatorname{sh}\left(z+i k \alpha_{j l}\right)}{(z \rightarrow-z)} \quad(\mathrm{sG}) .
$$

We proceed by obtaining and studying integral representations. In view of (3.1) and (3.3), we may rewrite $u(4.1)$ as

$$
u(z)=\exp (E(z))
$$

with

$$
E(z) \equiv 2 i \int_{0}^{\infty} \frac{d y}{y} \frac{\operatorname{sh}\left(a_{+}-b\right) y \operatorname{sh}\left(a_{-}-b\right) y}{\operatorname{sh} a_{+} y \operatorname{sh} a_{-} y} \sin 2 y z .
$$

Clearly, the integral converges absolutely provided

$$
|\operatorname{Im} z|<d\left(a_{+}, a_{-}, b\right) / 2,
$$

where

$$
d\left(a_{+}, a_{-}, b\right) \equiv a_{+}+a_{-}-\left|a_{+}-b\right|-\left|a_{-}-b\right|
$$

In particular, one has

$$
d\left(a_{+}, a_{-}, b\right)>a_{\delta} \Leftrightarrow\left(a_{+}, a_{-}, b\right) \in \mathscr{R}_{\delta},
$$

cf. (4.10). This bound amounts to the regularity of $u(z)$ in $\mathscr{R}_{\delta}$, viewed as a solution to (4.6): $u$ has no poles and zeros in the strip $|\operatorname{Im} z| \leqslant a_{\delta} / 2$ when $\left(a_{+}, a_{-}, b\right) \in \mathscr{R}_{\delta}$.

More generally, setting

$$
\mathscr{C} \equiv\left\{\left(a_{+}, a_{-}, b\right) \in \mathscr{H} \mid b \in\left(0, a_{+}+a_{-}\right)\right\},
$$


the representation (4.29) makes sense and holds true in a strip around the real $z$-axis if and only if the parameters belong to $\mathscr{C}$. Indeed, one easily verifies

$$
d\left(a_{+}, a_{-}, b\right)>0 \Leftrightarrow\left(a_{+}, a_{-}, b\right) \in \mathscr{C} .
$$

Observe that $\mathscr{R}_{+} \cup \mathscr{R}_{-}$is a proper subset of $\mathscr{C}$.

Letting $|\operatorname{Im} z|<a_{\delta} / 2$ and choosing parameters in $\mathscr{R}_{\delta}$, we can derive a second integral representation from Theorems II.3 and II.2, as applied to the A $\Delta \mathrm{E}$ (4.6). From (4.29) and (4.30) we read off that the minimum integer $k$ in Theorem II.3 equals 1 . Setting

$$
\phi_{\delta}(z) \equiv \ln \left(\frac{s_{-\delta}\left(z-i b+i a_{\delta} / 2\right) s_{-\delta}\left(z+i b-i a_{\delta} / 2\right)}{s_{-\delta}\left(z+i a_{\delta} / 2\right) s_{-\delta}\left(z-i a_{\delta} / 2\right)}\right)
$$

with $\ln$ real for $z$ real, we now deduce

$$
E(z)=\frac{1}{2 i a_{\delta}} \int_{-\infty}^{\infty} d x \phi_{\delta}(x) \operatorname{th} \frac{\pi}{a_{\delta}}(z-x), \quad\left(a_{+}, a_{-}, b\right) \in \mathscr{B}_{\delta}, \quad|\operatorname{Im} z|<a_{\delta} / 2 .
$$

(Indeed, both lhs and rhs vanish for $z=0$, and equality of derivatives is easily derived via (2.27) with $a \rightarrow a_{\delta}$ and $\phi(u) \rightarrow \phi_{\delta}^{\prime}(u)$.) Notice that the integral on the rhs converges absolutely for real $z$ and any $\left(a_{+}, a_{-}, b\right) \in \mathscr{H}$; even so, (4.37) is in general false for parameters not belonging to $\mathscr{B}_{\delta}$. Note also that for parameters in $\mathscr{B}_{+} \cap \mathscr{R}_{-}$one gets two different representations without manifest $a_{+} \leftrightarrow a_{-}$symmetry.

Using the identity (A42) we can rewrite (4.37) as

$$
E(z)=\frac{\operatorname{sh}\left(2 \pi z / a_{\delta}\right)}{i a_{\delta}} \int_{0}^{\infty} \frac{\phi_{\delta}(x) d x}{\operatorname{ch}\left(2 \pi z / a_{\delta}\right)+\operatorname{ch}\left(2 \pi x / a_{\delta}\right)}, \quad\left(a_{+}, a_{-}, b\right) \in \mathscr{R}_{\delta}, \quad|\operatorname{Im} z|<a_{\delta} / 2 .
$$

Combining this with (A43), (A44) and the Plancherel relation for the cosine transform, one recovers the symmetric representation (4.30).

We proceed by deriving yet another asymmetric representation for the $u$-function, in terms of an infinite product of gamma functions. (Somewhat surprisingly, this representation is not an easy consequence of (3.63), (3.64) and (3.67).) First, we introduce

$$
\gamma_{l}(\rho, g, s) \equiv \Gamma(s+1+l / \rho) \Gamma(-s+g+l / \rho) \Gamma(s+l / \rho) \Gamma(-s+1-g+l / \rho) /(s \rightarrow-s),
$$

where $l \in \mathbb{N}, \rho \in \mathbb{C}^{-}, g, s \in \mathbb{C}$. Fixing $l, g, s$ and taking $\rho>0$ and small enough, we may invoke (A45) to deduce

$$
\gamma_{l}(\rho, g, s)=\exp \left(4 \int_{0}^{\infty} \frac{d y}{y} \frac{\operatorname{sh}(g-1) y \operatorname{sh} 2 s y \operatorname{sh} g y}{\operatorname{sh} y} e^{-2 l y / \rho}\right) .
$$

This representation is well defined and valid for

$$
l \operatorname{Re}\left(\rho^{-1}\right)>|\operatorname{Reg}|+|\operatorname{Res}| .
$$

By virtue of (B18) it can be rewritten

$$
\gamma_{l}(\rho, g, s)=\exp \left(4 \int_{0}^{\infty} e^{-2 l t / \rho} f_{3}(g-1,2 s, g, t) d t\right) .
$$


Next, we assert that the function

$$
P(\rho, g, s) \equiv \lim _{N \rightarrow \infty} \prod_{l=1}^{N} \gamma_{l}(\rho, g, s)
$$

is well defined and meromorphic in $\mathrm{C}^{-} \times \mathrm{C}^{2}$. To prove this, we fix a compact $K \subset \mathrm{C}^{-} \times \mathrm{C}^{2}$ and put $w \equiv 2 l / \rho$. Letting $(\rho, g, s)$ vary over $K$, we can ensure (by taking $l \geqslant L$ with $L$ large enough) that the bound (B21) applies for a suitable $\chi \in(0, \pi / 2)$ and $R$ (depending on $K$ ). Thus we deduce that $\gamma_{l}$ is analytic on $K$ and satisfies

$$
\left|\gamma_{l}(\rho, g, s)-1\right| \leqslant C_{K} / l^{2}, \quad \forall(\rho, g, s) \in K, \quad \forall l \geqslant L .
$$

Consequently, the function $\Pi_{l=L}^{N} \gamma_{l}$ converges uniformly on $K$ to an analytic function for $N \rightarrow \infty$, and the assertion easily follows.

We claim that $u$ can be written

$$
u\left(a_{+}, a_{-}, b ; z\right)=\frac{\Gamma\left(\frac{i z}{a_{-}}+1\right) \Gamma\left(-\frac{i z}{a_{-}}+\frac{b}{a_{-}}\right)}{(z \rightarrow-z)} P\left(\frac{a_{-}}{a_{+}}, \frac{b}{a_{-}}, \frac{i z}{a_{-}}\right) .
$$

Since we already know that $u$ is meromorphic for $\left(a_{-} / a_{+}, b, z\right) \in \mathrm{C}^{-} \times \mathrm{C}^{2}$, we need only prove this for $z=x \in \mathbb{R}$ and parameters in $\mathscr{C}$ (4.34). To this end we show that the rhs is given by $\exp (E(x))$ (with $E(x)$ defined by (4.30)): Using (A45) and (4.40) we have (with $g \equiv b / a_{-}$)

$$
\begin{aligned}
& \frac{\Gamma\left(\frac{i x}{a_{-}}+1\right) \Gamma\left(-\frac{i x}{a_{-}}+g\right) \prod_{l=1}^{N} \gamma_{l}\left(\frac{a_{-}}{a_{+}}, g, \frac{i x}{a_{-}}\right)}{(x \rightarrow-x)} \\
& \quad=\exp \left(2 i \int_{0}^{\infty} \frac{d y}{y} \frac{\operatorname{sh}(1-g) y \sin \left(2 x y / a_{-}\right)}{\operatorname{sh} y}\left(e^{-g y}-2 \operatorname{sh} g y \sum_{l=1}^{N} \exp \left(-2 l y a_{+} / a_{-}\right)\right)\right) \\
& =\exp \left(i \int _ { 0 } ^ { \infty } \frac { d y } { y } \frac { \operatorname { s h } ( a _ { - } - b ) y \operatorname { s i n } ( 2 x y ) } { \operatorname { s h } a _ { - } y \operatorname { s h } a _ { + } y } \left(e^{-b y}\left(e^{a_{+} y}-e^{-a_{+} y}\right)\right.\right. \\
& \left.\left.\quad+\left(e^{-b y}-e^{b y}\right) e^{-a_{+} y}\left(1-e^{-2 a_{+} N y}\right)\right)\right) .
\end{aligned}
$$

A dominated convergence argument now shows that we may take $N \rightarrow \infty$ under the integral sign, yielding the limit $\exp (E(x))$, as claimed.

We conclude this subsection by deriving four distinct limits of the $u$-function, using parameters

$$
a_{+} \equiv \pi, a_{-} \equiv \beta \nu, b \equiv \beta \nu g, \quad \beta, \nu>0, \quad g \in \mathbb{R}
$$

First, we assert that

$$
\lim _{\beta \downarrow 0} u(\pi, \beta \nu, \beta \nu g ; \beta p)=\frac{\Gamma\left(\frac{i p}{\nu}+1\right) \Gamma\left(-\frac{i p}{\nu}+g\right)}{(p \rightarrow-p)} \quad\left(\mathrm{II}_{\mathrm{nr}} \text { limit }\right),
$$

where the limit is mero-uniform in $p$. To show this, we use (4.1), (3.22), (3.24) and (3.69) to write 


$$
u(\pi, \beta \nu, \beta \nu g ; \beta p)=\frac{H(\rho ; p / \nu-i g+i / 2) H(\rho ;-p / \nu-i / 2)}{(p \rightarrow-p)}, \quad \rho \equiv \beta \nu / \pi
$$

Then the assertion follows from (3.72).

The formula (4.48) can be interpreted as the (nonrelativistic) $\mathrm{II}_{\mathrm{nr}}$ limit of the (relativistic) $\mathrm{II}_{\text {rel }} S$-matrix, cf. Ref. 1, Eq. (3.45). It can also be derived from the product representation (4.45). Indeed, one has

$$
\lim P(\rho, g, s)=1
$$

uniformly for $g, s$ in a fixed compact $B \subset C^{2}$. To verify this, note first that $\gamma_{l}(\rho, g, s)(4.39)$ is analytic in $B$ for $\rho>0$ small enough, and given by (4.40). From this representation it follows that $\gamma_{l}(\rho, g, s)$ converges to 1 as $\rho \downarrow 0$, uniformly for $(g, s) \in B$. Next, observe that for $\rho \leqslant \epsilon$ (with $\epsilon$ depending only on $B$ ) one may use (4.42) and the bound (B21) with $w \equiv 2 l / \rho$ to deduce

$$
\left|\gamma_{l}(\rho, g, s)-1\right| \leqslant C_{B} \rho^{2} / l^{2} \leqslant C_{B} \epsilon^{2} / l^{2}, \quad \forall(g, s) \in B, \quad \forall l \in \mathbb{N}^{*} .
$$

Clearly, this bound suffices to dominate the $l$-dependence, so one infers $P \rightarrow 1$, uniformly on $B$.

The next limit amounts to taking the $\mathrm{I}_{\text {rel }}$ limit of the dual $\mathrm{II}_{\text {rel }} S$-matrix, cf. Ref. 1: We claim

$$
\lim _{\beta \downarrow 0} u(\pi, \beta \nu, \beta \nu g ; \nu x)=\exp (i \pi(1-g)), \quad x \in \mathscr{B}_{0} \quad\left(\mathrm{I}_{\mathrm{rel}} \text { limit }\right),
$$

where the limit is uniform on compacts of $\mathscr{B}_{0}$ (2.73). Before proving this, let us note that the restriction on $x$ is essential: for Re $x<0$ one obtains the complex conjugate phase factor by virtue of (4.3). (For $g \notin \mathbb{Z}$, the poles and zeros of $u$ become dense on the imaginary axis as $\beta \downarrow 0$, cf. (4.1) and Prop. III.3.) Observe also that the phase amounts to a limit of the phase in (4.12).

To prove (4.52), we use the product representation (4.45) and several results from Appendix B. First, we handle the prefactor

$$
Q_{\beta}(g, x) \equiv \frac{\Gamma(i x / \beta+1) \Gamma(-i x / \beta+g)}{(x \rightarrow-x)} .
$$

It can be rewritten

$$
Q_{\beta}(g, x)=e^{i \pi(1-g)}\left(\frac{\Gamma\left(w_{+}+1\right)}{\Gamma\left(w_{+}+g\right)} e^{(g-1) \ln w_{+}}\right)\left(\frac{\Gamma\left(w_{-}+g\right)}{\Gamma\left(w_{-}+1\right)} e^{(1-g) \ln w_{-}}\right), \quad w_{ \pm} \equiv \pm \frac{i x}{\beta} .
$$

Using (B23) to rewrite the functions in brackets, and letting $x$ vary over a fixed compact $K \subset \mathscr{R}_{0}$, we now exploit the bound (B20). First, taking $R=1+|g|$ and $\chi=\pi / 4$ (say), one can ensure $w_{+}, w_{-} \in S_{R, \chi}$ for all $x \in K$ by choosing $\beta$ small enough. Then it follows from (B20) that

$$
\lim _{\beta \downarrow 0} Q_{\beta}(g, x)=\exp (i \pi(1-g))
$$

uniformly for $x \in K$. (This may be viewed as the $\mathrm{II}_{\mathrm{nr}} \rightarrow \mathrm{I}_{\mathrm{nr}} S$-matrix limit, cf. Ref. 1, Eq. (3.45).)

It remains to prove

$$
\lim _{\rho \downarrow 0} P(\rho, g, i y / \rho)=1
$$

uniformly on compacts of $\{$ Rey $>0\}$. To this end we first use (4.39) and (B23) to write 


$$
\begin{aligned}
\gamma_{l}(\rho, g, i y / \rho)= & \exp \left(\mathscr{L}_{2}((i y+l) / \rho, 1, g)+\mathscr{L}_{2}((-i y+l) / \rho, g, 1)+\mathscr{L}_{2}((i y+l) / \rho, 0,1-g)\right. \\
& \left.+\mathscr{L}_{2}((-i y+l) / \rho, 1-g, 0)\right) .
\end{aligned}
$$

Next, we let $y$ vary over a compact $K \subset \mathscr{R}_{0}$, and use the bound (B20) in the same way as before to infer that $\gamma_{l} \rightarrow 1$ for $\rho \downarrow 0$, uniformly on $K$.

As a consequence, (4.56) will follow provided we can supply a bound controlling the interchange of limits $N \rightarrow \infty$ and $\rho \downarrow 0$. Now the estimate (B20) is not sufficiently strong, since it only leads to $1 / l$-decrease of $\left|\gamma_{l}-1\right|$, and the sequence $(1,1 / 2,1 / 3, \ldots)$ is not in $l^{1}$. But we can obtain a suitable bound by combining the representation (4.42) with the estimates (B21) and (B26), as follows.

We begin by observing that (4.42) and (B15) entail

$$
\gamma_{l}(\rho, g, i y / \rho)=\exp \left(4 \mathscr{L}_{3}(2 l / \rho, g-1, g, 2 i y / \rho)\right) .
$$

Letting $y$ vary over $K$ and choosing $\rho \in(0, \epsilon]$ with $\epsilon$ small enough, we may take $r_{3}=c_{K} / \rho$ in the bound (B21) on $\mathscr{L}_{3}$. Choosing now $\chi=0, R=\left(c_{K}+1\right) / \rho$ and $L>\left(c_{K}+1\right) / 2$, we deduce

$$
\left|\mathscr{L}_{3}\left(\frac{2 l}{\rho}, g-1, g, \frac{2 i y}{\rho}\right)-\frac{i \rho y g(g-1)}{l^{2}}\right| \leqslant \frac{\rho^{3}}{4 l^{2}} C_{3}, \quad \rho \in(0, \epsilon], \quad l \geqslant L, \quad y \in K .
$$

Next, we use the bound (B26) to majorize the rhs of (4.59) by $C \rho / l^{2}$. By dominated convergence, this suffices to conclude that the function $\prod_{l=L}^{\infty} \gamma_{l}$ converges to 1 as $\rho \downarrow 0$, uniformly on $K$. Since we have already shown that $\gamma_{l} \rightarrow 1$ uniformly on $K$ for all $l \geqslant 1$, we may now deduce (4.56). (Notice that (4.58) and (B21) are not adequate for showing $\gamma_{l} \rightarrow 1$ for small $l$; this is why we used (4.57) and (B19).)

Alternatively, (4.52) can be derived as a corollary of Prop. III.7. Indeed, from (4.1) we have

$$
u(\pi, a, a g ; z)=\frac{G(\pi, a ; z+i \pi / 2+i a(1 / 2-g))}{G(\pi, a ; z+i \pi / 2-i a / 2)} \cdot \frac{G(\pi, a ; z-i \pi / 2+i a(g-1 / 2))}{G(\pi, a ; z-i \pi / 2+i a / 2)}
$$

Thus, we may use (3.91) with $\operatorname{Re} z>0$ to deduce the limit (4.52).

It is of interest to reconsider this limit in the setting of Theorem II.4. Choosing, e.g., $g$ $\in(1 / 2,1)$, one can take $f_{a}(z)$ equal to $\partial_{z} \ln u(\pi, a, a g ; z)$; letting $a \rightarrow 0$, one gets $s_{m}(a) \rightarrow 0$ and $f_{a}(z) \rightarrow 0$ uniformly on compacts in the left and right half planes. Even so, $f_{a}(z)$ does not remain bounded near the origin, since $u(z)$ has distinct limits in the left and right half planes.

We continue by obtaining a third limit of the $u$-function, keeping the parameters (4.47), but now taking $b$ fixed while letting $\beta \downarrow 0$. Specifically, we claim

$$
\begin{gathered}
\lim _{\beta \downarrow 0} \exp \left(-\frac{2 i p}{\nu} \ln \left(\frac{\beta}{2 \sin b}\right)\right) u(\pi, \beta \nu, b ; \beta p)=\frac{\Gamma\left(\frac{i p}{\nu}+1\right)}{(p \rightarrow-p)} \exp \left(\frac{2 i p}{\nu} \ln (2 \nu)\right), \\
b \in(0, \pi) \quad\left(\mathrm{VI}_{\mathrm{nr}} \text { limit }\right),
\end{gathered}
$$

where the limit is mero-uniform. The function on the rhs may be viewed as the (nonrelativistic Toda) $\mathrm{VI}_{\mathrm{nr}} S$-matrix, cf. Ref. 1, Eq. (3.45). The limiting transition $\mathrm{II}_{\mathrm{rel}} \rightarrow \mathrm{VI}_{\mathrm{nr}}$ is readily controlled at the level of the Poisson commuting classical Hamiltonians, cf. the paragraph containing Eq. (3.87) in Ref. 2. Formally, it also holds true for the corresponding quantum Hamiltonians. The $S$-matrix limit (4.61) agrees with the obvious conjecture that the limit holds true for the suitably normalized (reduced $N=2$ ) eigenfunctions; the plane wave factor on the lhs reflects the diverging position shift (3.87) in Ref. 2.

To prove (4.61), we begin by observing that 


$$
\lim _{g \uparrow \infty} \exp \left(\frac{2 i p}{\nu} \ln g\right) \frac{\Gamma\left(-\frac{i p}{\nu}+g\right)}{(p \rightarrow-p)}=1
$$

uniformly on $p$-compacts. (This limit amounts to the $\mathrm{II}_{\mathrm{nr}} \rightarrow \mathrm{VI}_{\mathrm{nr}} S$-matrix limit, cf. Ref. 1, Eq. (3.45), and the paragraph containing Eq. (2.116) in Ref. 2.) Indeed, this follows from (B23) and (B20) (taking $w=g$ ) in a by now familiar way. As a result, (4.61) will follow once we show

$$
\lim _{\rho \downarrow 0} P(\rho, b / \pi \rho, s)=\exp \left(2 \sin \left(\frac{b}{\sin b}\right)\right), \quad b \in(0, \pi),
$$

uniformly on $s$-compacts.

To prove (4.63), we write

$$
\begin{aligned}
\gamma_{l}(\rho, b / \pi \rho, s)= & \exp \left(\mathscr{L}_{2}(l / \rho, s+1,-s+1)\right) \exp \left(\mathscr{L}_{2}(l / \rho, s,-s)\right) \\
& \times \exp \left(\mathscr{L}_{2}((l \pi+b) / \pi \rho,-s, s)\right) \\
& \times \exp \left(\mathscr{L}_{2}((l \pi-b) / \pi \rho,-s+1, s+1)\right) \exp \left(-2 s \ln \left(1-b^{2} / l^{2} \pi^{2}\right)\right) .
\end{aligned}
$$

Since $b \in(0, \pi)$, we have $l \pi \pm b>0$, and so we conclude using (B20)

$$
\lim _{\rho \downarrow 0} \gamma_{l}(\rho, b / \pi \rho, s)=\exp \left(-2 s \ln \left(1-b^{2} / l^{2} \pi^{2}\right)\right)
$$

uniformly on $s$-compacts. Now from (A23)-(A25) [with $\alpha=0$, cf. (A28)] one derives the wellknown identity

$$
\frac{\sin b}{b}=\prod_{l=1}^{\infty}\left(1-\frac{b^{2}}{l^{2} \pi^{2}}\right)
$$

Using this on the rhs of (4.63) and comparing with (4.65), we infer that we need only supply a bound that is sufficiently strong to render the interchange of limits legitimate.

The bound (B20) leads to an $O\left(l^{-1}\right)$-majorization, so it is not strong enough. Just as in the previous case, we will now derive on $O\left(l^{-2}\right)$ estimate (for $l$ sufficiently large) by combining (B21) and (B26). To this purpose we observe that we may write

$$
\gamma_{l}(\rho, b / \pi \rho, s)=\exp \left(4 \mathscr{L}_{3}(2 l / \rho,-1+b / \pi \rho, 2 s, b / \pi \rho)\right),
$$

cf. (4.42) and (B 15). For. $s$ in a compact $B \subset \mathrm{C}$ and $\rho \in(0, \epsilon]$ with $\epsilon$ small enough, we can take $r_{3}=c_{B} / \rho$ in (B21). Choosing then $\chi=0, R=\left(c_{B}+1\right) / \rho$ and $L>\left(c_{B}+1\right) / 2$, we obtain

$$
\left|\mathscr{L}_{3}\left(\frac{2 l}{\rho},-1+\frac{b}{\pi \rho}, 2 s, \frac{b}{\pi \rho}\right)-\frac{s b(b-\pi \rho)}{2 l^{2} \pi^{2}}\right| \leqslant \frac{\rho^{3}}{4 l^{2}} C_{3}, \quad \rho \in(0, \epsilon], \quad l \geqslant L, \quad s \in B .
$$

Using now (B26), we obtain an upper bound $C / l^{2}$ on the rhs. As before, this suffices to conclude that (4.63) holds true. The upshot is that the proof of (4.61) is now complete.

As a corollary of (4.61), we can obtain the integral

$$
\frac{\Gamma(1+i z)}{\Gamma(1-i z)}=\exp \left(\frac{\operatorname{sh} 2 \pi z}{2 i} \int_{0}^{\infty} \frac{d t}{\operatorname{ch} 2 \pi z+\operatorname{ch} \pi t} \ln \left(\frac{4}{t^{2}+1}\right)\right), \quad|\operatorname{Im} z|<\frac{1}{2}
$$


Indeed, combining the integral

$$
\operatorname{sh} 2 \pi z \int_{0}^{\infty} \frac{d t}{\operatorname{ch} 2 \pi z+\operatorname{ch} \pi t}=2 z, \quad|\operatorname{Im} z|<\frac{1}{2}
$$

(which results from (A43), e.g.), with (4.29), (4.38) and (4.36), we obtain

$$
\begin{aligned}
& \exp \left(-2 i z \ln \left(\frac{\beta}{\sin b}\right)\right) u(\pi, \beta, b ; \beta z) \\
& =\exp \left(\frac{\operatorname{sh} 2 \pi z}{2 i} \int_{0}^{\infty} \frac{d t}{\operatorname{ch} 2 \pi z+\operatorname{ch} \pi t} \ln \left(\frac{\operatorname{sh}^{2} \beta t / 2+\sin ^{2}(b-\beta / 2)}{\operatorname{sh}^{2} \beta t / 2+\sin ^{2} \beta / 2} \cdot \frac{\beta^{2}}{\sin ^{2} b}\right)\right),
\end{aligned}
$$

where $\beta \in(0, b / 2), b \in(0, \pi),|\operatorname{Im} z|<1 / 2$. A straightforward dominated convergence argument now shows that the rhs of (4.71) converges to the rhs of (4.69) for $\beta \downarrow 0$. From (4.61) we see that the lhs converges to the lhs of (4.69), so (4.69) results.

Finally, we obtain a limit that may be viewed as the classical limit of the quantum $\mathrm{II}_{\mathrm{rel}}$ $S$-matrix. To this end we introduce

$$
L_{\hbar}(p) \equiv i \hbar \ln u(\pi, \hbar / \lambda, b ; p), \quad(\lambda, b, p) \in(0, \infty) \times(0, \pi) \times \mathscr{R}_{0},
$$

with $\ln u \rightarrow 0$ for $p \rightarrow 0, \hbar>0$ denoting Planck's constant. We now claim that

$$
\lim _{\hbar \rightarrow 0} \partial_{p} L_{\hbar}(p)=\lambda \ln \left(\frac{\operatorname{sh}(p+i b) \operatorname{sh}(p-i b)}{\operatorname{sh}^{2} p}\right) \quad \text { (classical limit) }
$$

uniformly on compact subsets of the right half plane $\mathscr{R}_{0}$, with $\ln$ real valued for $p>0$. (The rhs amounts to the classical $\mathrm{II}_{\text {rel }}$ phase shift, cf. Ref. 1, Eq. $(2.75)$ with $\beta=1$.)

To prove this claim, we substitute $a g \rightarrow b$ in (4.60) and use (3.83) and (3.84) to write

$$
\begin{aligned}
i a \ln u(\pi, a, b ; z)= & -D_{a}(z+i \pi / 2-i b)-D_{a}(z-i \pi / 2+i b)+D_{a}(z+i \pi / 2)+D_{a}(z-i \pi / 2) \\
& -a d_{a}(1 / 2,0 ; z+i \pi / 2-i b)-a d_{a}(-1 / 2,0 ; z-i \pi / 2+i b) \\
& +a d_{a}(-1 / 2,0 ; z+i \pi / 2)+a d_{a}(1 / 2,0 ; z-i \pi / 2) .
\end{aligned}
$$

Taking $a \rightarrow 0$, the limit of (4.74) exists uniformly on compacts in $\mathscr{k}_{0}$ by virtue of (3.85) and (3.86). Taking $z$-derivatives, one readily obtains a limit that amounts to (4.73).

\section{B. The elliptic case}

The elliptic scattering function is defined in terms of the elliptic $G$-function from Subsection III B via (4.1). In view of Prop. III.11, this yields a function that is meromorphic in $r, a_{+}, a_{-}, b$ and $z$, as long as $a_{+} r$ and $a_{-} r$ stay in the right half plane. We shall from now on restrict the parameters to

$$
\mathscr{E} \equiv\left\{\left(r, a_{+}, a_{-}, b\right) \mid r>0,\left(a_{+}, a_{-}, b\right) \in \mathscr{H}\right\},
$$

cf. (4.5). By virtue of Prop. III.9 the elliptic $u$-function is periodic in $z$ with primitive period $\pi / r$; moreover, it satisfies (4.2), (4.3), and

$$
\begin{gathered}
u\left(2 r, a_{+}, a_{-}, b ; z\right)=u\left(r, a_{+}, a_{-}, b ; z\right) u\left(r, a_{+}, a_{-}, b ; z-\pi / 2 r\right) \\
u\left(\lambda^{-1} r, \lambda a_{+}, \lambda a_{-}, \lambda b ; \lambda z\right)=u\left(r, a_{+}, a_{-}, b ; z\right), \quad \lambda \in(0, \infty) .
\end{gathered}
$$


Recalling (3.96)-(3.100), and using also (2.91), we see that $u$ solves the A $\Delta \mathrm{Es}$

$$
\frac{u\left(z+i a_{\delta} / 2\right)}{u\left(z-i a_{\delta} / 2\right)}=\exp \left(2 r\left(a_{\delta}-b\right)\right) \frac{s_{-\delta}\left(z-i b+i a_{\delta} / 2\right) s_{-\delta}\left(z+i b-i a_{\delta} / 2\right)}{s_{-\delta}\left(z+i a_{\delta} / 2\right) s_{-\delta}\left(z-i a_{\delta} / 2\right)}
$$

It now follows just as in the hyperbolic case that $u$ is a regular solution to (4.78) if and only if $\left(a_{+}, a_{-}, b\right) \in \mathscr{R}_{\delta}$. Since $u$ is $\pi / r$-periodic in $z$, the latter restriction also ensures that $u$ is the unique minimal solution satisfying (4.11). Furthermore, with (4.6) replaced by (4.78), the remark below (4.12) applies verbatim to the elliptic case.

Using (3.100) and (2.91) we now obtain the analog of (4.13):

$$
\frac{u\left(b+a_{-\delta} ; z\right)}{u(b ; z)}=-e^{2 i r z} \frac{s_{\delta}(z+i b)}{s_{\delta}(z-i b)} .
$$

To simplify the iterations of these A $\Delta$ Es, we use the formula

$$
\frac{s\left(r, a ; z_{+}+i n a\right)}{s\left(r, a ; z_{-}-i n a\right)}=e^{-2 i r n\left(z_{+}+z_{-}\right)} \frac{s\left(r, a ; z_{+}\right)}{s\left(r, a ; z_{-}\right)}, \quad n \in \mathbb{N},
$$

which follows from (2.91). Then we obtain once more the relation (4.14), but now with an extra factor $\exp \left(2 \operatorname{irz}\left(k_{+}+k_{-}-2 k_{+} k_{-}\right)\right)$on the rhs. Noting the elliptic analog

$$
u\left(r, a_{+}, a_{-}, a_{+} ; z\right)=u\left(r, a_{+}, a_{-}, a_{-} ; z\right)=1
$$

of (4.16), we deduce the elliptic analog

$$
u\left(r, a_{+}, a_{-}, 0 ; z\right)=-e^{-2 i r z}
$$

of (4.17) and, more generally, the explicit formula (4.18), with (4.20) replaced by

$$
c_{k, l} \equiv(-)^{k+l+1} \exp (2 \operatorname{irz}(k+l-2 k l-1)), \quad k, l \in \mathbb{Z}
$$

It is clear that the symmetry property (4.21) continues to hold in the elliptic case. Moreover, it leads again to the relation (4.22) between $u(-b ; z)$ and $u(b ; z)$. Next, we note that (4.23) still holds true, since the elliptic $G$-function satisfies the multiplication formula (3.25). Hence, (4.24) follows as before. Using the $\mathrm{A} \Delta \mathrm{Es}(3.100)$ and (2.91) we then obtain as the analogs of (4.25) and (4.26)

$$
\begin{aligned}
u\left(r, \frac{a_{+}}{M}, a_{-}, b ; z\right)= & (-)^{M-1} \exp \left(\operatorname{ir}(M-1)\left(2 M z+i a_{+}-i b\right)\right) \\
& \cdot \prod_{k=0}^{M-1} u\left(r, a_{+}, a_{-}, b ; z+i k \frac{a_{+}}{M}\right) \prod_{j=1}^{M-1} \frac{s_{-}\left(z+i j a_{+} / M\right)}{s_{-}\left(z-i b+i j a_{+} / M\right)}
\end{aligned}
$$

and

$$
\begin{aligned}
& u\left(r, \frac{a_{+}}{M}, a_{-}, b ; z\right)=(-)^{M-1} \exp \left(\operatorname{ir}(M-1)\left(2 M z-i a_{+}+i b\right)\right) \\
& \cdot \prod_{k=0}^{M-1} u\left(r, a_{+}, a_{-}, b ; z-i k \frac{a_{+}}{M}\right)^{M-1} \prod_{j=1}^{M-\left(z+i b-i j a_{+} / M\right)} \\
& s_{-}\left(z-i j a_{+} / M\right)
\end{aligned}
$$


Once more, $a_{+} \leftrightarrow a_{-}$symmetry can now be used to obtain four distinct representations for $u\left(r, a_{+} / M, a_{-} / N, b ; z\right)$ in terms of $u\left(r, a_{+}, a_{-}, b ; z\right)$ and $s$-quotients.

The choices $b=a_{+} / 2$ or $b=a_{-} / 2$ yield the XYZ soliton-soliton $S$-matrix. Thus it follows from (4.18) and (4.83) that the counterpart of (4.28) reads

$$
\begin{aligned}
u\left(r, \pi, \alpha_{j l}, \pi / 2 ; z\right)= & \exp (2 i r z(l-j+2 l j-1)) \\
& \cdot \prod_{m=1}^{j} \frac{s\left(r, \alpha_{j l} ; z+i m \pi\right)}{(z \rightarrow-z)} \prod_{k=1}^{l-1} \frac{s\left(r, \pi ; z+i k \alpha_{j l}\right)}{(z \rightarrow-z)} \quad(\mathrm{XYZ}) .
\end{aligned}
$$

Next, we use (4.1), (3.92) and (3.3) to obtain

$$
u(z)=\exp (E(z))=\exp \left(2 i \sum_{n=1}^{\infty} \frac{\operatorname{sh}\left(a_{+}-b\right) n r \operatorname{sh}\left(a_{-}-b\right) n r}{n \operatorname{sh} a_{+} n r \operatorname{sh} a_{-} n r} \sin 2 n r z\right)
$$

The series converges absolutely if and only if (4.31) holds true. As before, regularity of $u(z)$ for parameters in $\mathscr{R}_{\delta}$ can be read off from (4.33). Furthermore, the series representation (4.87) is valid for real $z$ iff the parameters belong to the convergence region (4.34).

Choosing $\left(a_{+}, a_{-}, b\right) \in \mathscr{R}_{\delta}$ and introducing

$$
\phi_{\delta}(z) \equiv \ln \left(\frac{s_{-\delta}\left(z-i b+i a_{\delta} / 2\right) s_{-\delta}\left(z+i b-i a_{\delta} / 2\right)}{s_{-\delta}\left(z+i a_{\delta} / 2\right) s_{-\delta}\left(z-i a_{\delta} / 2\right)}\right)+2 r\left(a_{\delta}-b\right)
$$

with $\ln$ real for $z$ real, we can combine (4.78) and (4.87) to deduce that $\phi_{\delta}(z)$ satisfies the assumptions (2.100)-(2.102) of Theorem II.5. Therefore, (2.107) yields

$$
E(z)=\frac{1}{2 i \pi} \int_{-\pi / 2 r}^{\pi / 2 r} d y \phi_{\delta}(y) K\left(r, a_{\delta} ; z-y\right), \quad\left(a_{+}, a_{-}, b\right) \in \mathscr{R}_{\delta}, \quad|\operatorname{Im} z|<\frac{a_{\delta}}{2} .
$$

This representation amounts to the elliptic counterpart of (4.37). Once more, the restriction on the parameters is essential (though boundary points of $\mathscr{B}_{\delta}$ belonging to $\mathscr{H}$ (4.5) can be allowed, of course).

The product representation (3.117) for the elliptic $G$-function can be combined with (4.1) to yield

$$
\begin{aligned}
u\left(r, a_{+}, a_{-}, b ; z\right)= & \prod_{m, n=1}^{\infty} \frac{\left(1-2 q_{+}^{2 m-1} q_{-}^{2 n-1} e^{-2 i r z} \operatorname{ch}\left(b-\left(a_{+}+a_{-}\right) / 2\right)+q_{+}^{4 m-2} q_{-}^{4 n-2} e^{-4 i r z}\right)}{(z \rightarrow-z)} \\
& \cdot \frac{\left(1-2 q_{+}^{2 m-1} q_{-}^{2 n-1} e^{2 i r z} \operatorname{ch}\left(a_{+}-a_{-}\right) / 2+q_{+}^{4 m-2} q_{-}^{4 n-2} e^{4 i r z}\right)}{(z \rightarrow-z)}, \quad q_{\delta} \equiv e^{-a_{\delta} r}
\end{aligned}
$$

From this product representation one can read off meromorphy and pole/zero properties of $u(z)$. Notice that it is manifestly symmetric in $a_{+}, a_{-}$, in contradistinction to the product representation (4.45) for the hyperbolic $u$-function.

We proceed by deriving four limits of the $u$-function. First, we observe that

$$
\lim _{r \downarrow 0} u\left(r, a_{+}, a_{-}, b ; z\right)=u_{\mathrm{hyp}}\left(a_{+}, a_{-}, b ; z\right) \quad\left(\mathrm{II}_{\mathrm{rel}} \text { limit }\right),
$$


where the limit is mero-uniform. (Here, $u_{\text {hyp }}$ denotes the $u$-function from Subsection IV A.) Indeed, in the definition (4.1) of the elliptic $u$-function we may replace the elliptic $G$-functions by $G_{\text {ren }}$-functions, cf. (3.123). Then (4.91) is a consequence of Prop. III.12.

Second, we assert that the limit

$$
\lim _{A \uparrow \infty} u(r, a, A, b ; z)=u_{\text {trig }}(r, a, b ; z) \quad\left(\hat{\mathrm{III}}_{\mathrm{rel}} \text { limit }\right)
$$

exists mero-uniformly. (Here, $u_{\text {trig }}$ denotes the $u$-function studied in the next subsection.) To prove this, we use (4.1) and (3.22) to write

$$
u(r, a, A, b ; z)=\frac{G(r, a, A ; z+i b-i a / 2-i A / 2) G(r, a, A ;-z+i a / 2-i A / 2)}{G(r, a, A ;-z+i b-i a / 2-i A / 2) G(r, a, A ; z+i a / 2-i A / 2)} .
$$

Invoking now (3.139), we obtain the mero-uniform limit

$$
\lim _{A \uparrow \infty} u(r, a, A, b ; z)=\frac{G(r, a ; z+i b-i a / 2) G(r, a ;-z+i a / 2)}{G(r, a ;-z+i b-i a / 2) G(r, a ; z+i a / 2)},
$$

which amounts to (4.92), cf. (4.100) below.

Third, fixing $g \in \mathbb{R}$, we claim that

$$
\lim _{a \downarrow 0} u(r, A, a, a g ; z)=\exp ((1-g)(i \pi-2 i r z)), \quad z \in \mathscr{R}_{r} \quad\left(\hat{I} V_{\mathrm{nr}} \text { limit }\right)
$$

uniformly on compacts in the period strip $\mathscr{R}_{r}$ (2.113). Indeed, from (4.93) and (3.138) we obtain

$$
\lim _{a \downarrow 0} u(r, A, a, a g ; z)=\exp ((1-g) \ln (R(r, A ;-z-i A / 2) / R(r, A ; z-i A / 2))
$$

uniformly on compacts of $\mathscr{R}_{r}$. Now the limit (4.95) easily results from (3.93).

We continue by examining this result in the setting of Subsection II C. Taking $g \in[1,2]$ and $a \in(0, A / 4]$, it entails that Theorem II.7 applies to $f_{a}(z) \equiv \ln u(r, A, a, a g ; z)$. In this case $f_{a}^{\prime}(z)$ converges to the constant $2 i r(g-1)$, uniformly on compacts $K \subset \mathscr{R}_{r}$, but $f_{a}^{\prime}(z)$ diverges near $z=0$ as $a \rightarrow 0$. Indeed, the $\pi / r$-periodic function $f_{a}(x), x \in \mathbb{R}$, converges pointwise to a $\pi / r$-periodic function $f(x)$ that has unequal limits for $x \downarrow 0$ and $x \uparrow \pi / r$ (unless $g=1$, of course). Notice in this connection that it does not follow from the above that $f_{a}(z)$ remains bounded in the strip $|\operatorname{Im} z| \leqslant a / 2$ as $a \rightarrow 0$; we do not know whether this holds true.

We conclude this subsection by deriving the generalization of the classical limit (4.73). Thus we define

$$
L_{\hbar}(z) \equiv i \hbar \ln u(r, A, \hbar / \lambda, b ; z), \quad(r, \lambda, b, z) \in(0, \infty)^{2} \times(0, A) \times \mathscr{B}_{r},
$$

with $\ln u \rightarrow 0$ for $z \rightarrow 0$ and $\hbar>0$ Planck's constant. Then we have

$$
\lim _{\hbar \rightarrow 0} \partial_{z} L_{\hbar}(z)=\lambda \ln \left(e^{-2 r b} \frac{s(r, A ; z+i b) s(r, A ; z-i b)}{s(r, A ; z)^{2}}\right) \quad \text { (classical limit) }
$$

uniformly on an arbitrary compact $K \subset \mathscr{B}_{r}$, with In real for $z \in(0, \pi / r)$. infer

To prove this assertion, we exploit the obvious generalization of (4.74) and Prop. III.13 to 


$$
\lim _{a \downarrow 0} i a \partial_{z} \ln u(r, A, a, b ; z)=\ln \left(\frac{R(r, A ; z+i A / 2-i b) R(r, A ; z-i A / 2+i b)}{R(r, A ; z+i A / 2) R(r, A ; z-i A / 2)}\right)
$$

uniformly on $K$. Using (3.93) and (2.91), we see that this limit amounts to (4.98). Notice that the limit can be understood from Theorem II.7 and (4.78), with $a \ln u(z)$ playing the role of $f_{a}(z)$.

\section{The trigonometric case}

The trigonometric scattering function is defined by

$$
u(r, a, b ; z) \equiv \frac{G(z+i b-i a / 2) G(-z+i a / 2)}{G(-z+i b-i a / 2) G(z+i a / 2)}
$$

with $G(z) \equiv G(r, a ; z)$ denoting the trigonometric $G$-function (3.140). From the corresponding product representation

$$
u(r, a, b ; z)=\prod_{m=1}^{\infty} \frac{\left(1-q^{2 m-2} e^{-2 r b-2 i r z}\right)\left(1-q^{2 m} e^{2 i r z}\right)}{\left(1-q^{2 m-2} e^{-2 r b+2 i r z}\right)\left(1-q^{2 m} e^{-2 i r z}\right)}, \quad q \equiv e^{-a r},
$$

we read off that $u$ admits analytic continuation to a function that is meromorphic in $r, a, b$ and $z$, provided $a r$ stays in the right half plane. However, in the sequel we restrict the parameters to

$$
\mathscr{T} \equiv\{(r, a, b) \mid r>0, a>0, b \in \mathbb{R}\} .
$$

As before, this restriction entails $|u(z)|=1$ for real $z$.

Obviously, $u$ is periodic in $z$ with primitive period $\pi / r$; it also satisfies (4.3) and the relations

$$
\begin{gathered}
u(2 r, a, b ; z)=u(r, a, b ; z) u(r, a, b ; z-\pi / 2 r), \\
u\left(\lambda^{-1} r, \lambda a, \lambda b ; \lambda z\right)=u(r, a, b ; z), \quad \lambda \in(0, \infty) .
\end{gathered}
$$

From (2.90) and (4.78) [or directly from (4.100) and (3.142)] we deduce that $u$ satisfies the $\mathrm{A} \Delta \mathrm{E}$

$$
\frac{u(z+i a / 2)}{u(z-i a / 2)}=\exp (2 r(a-b)) \frac{\sin r(z-i b+i a / 2) \sin r(z+i b-i a / 2)}{\sin r(z+i a / 2) \sin r(z-i a / 2)} .
$$

Clearly, this $\mathrm{A} \Delta \mathrm{E}$ is regular unless $b=a / 2$. Now from the product representation (4.101) we see that $u(r, a, b ; z)$ may be viewed as the unique minimal solution to (4.105) that obeys (4.11), provided the parameters belong to the regularity region

$$
\mathscr{R} \equiv\{(r, a, b) \in \mathscr{T} \mid b \in(a / 2, \infty)\} .
$$

Next, we use (4.101) to conclude

$$
\frac{u(b+a ; z)}{u(b ; z)}=-e^{2 i r z} \frac{\sin r(z+i b)}{\sin r(z-i b)} .
$$

(Alternatively, this follows from (4.79) by taking a limit.) By iteration this gives rise to (taking $k \in \mathbb{Z})$

$$
\frac{u(b+k a ; z)}{u(b ; z)}=e^{2 i r k z} \prod_{j=1}^{|k|} \frac{\sin r(z+i(k /|k|)(b-a / 2)+i a(j-1 / 2))}{(z \rightarrow-z)} .
$$


Now from the product representation (4.101) we read off

$$
\begin{gathered}
u(r, a, a ; z)=1, \\
u(r, a, 0 ; z)=-e^{-2 i r z},
\end{gathered}
$$

and so (4.108) entails

$$
u(r, a, k a ; z)=(-)^{k+1} e^{2 i r(k-1) z} \prod_{j=1}^{|k|} \frac{\sin r(z+i a(j-\theta(k))}{(i \rightarrow-i)}, \quad k \in \mathbb{Z},
$$

with $\theta(k)$ defined by (4.19).

The trigonometric specializations of the relations (4.84) and (4.85) read

$$
\begin{aligned}
u\left(r, \frac{a}{M}, b ; z\right)= & (-)^{M-1} \exp (\operatorname{ir}(M-1)(2 M z+i a-i b)) \\
& \cdot \prod_{k=0}^{M-1} u\left(r, a, b ; z+i k \frac{a}{M}\right) \prod_{j=1}^{M-1} \frac{\sin r(z+i j a / M)}{\sin r(z-i b+i j a / M)}
\end{aligned}
$$

and

$$
\begin{aligned}
u\left(r, \frac{a}{M}, b ; z\right)= & (-)^{M-1} \exp (\operatorname{ir}(M-1)(2 M z-i a+i b)) \\
& \cdot \prod_{k=0}^{M-1} u\left(r, a, b ; z-i k \frac{a}{M}\right) \prod_{j=1}^{M-1} \frac{\sin r(z+i b-i j a / M)}{\sin r(z-i j a / M)}
\end{aligned}
$$

Of course, these formulas can also be verified directly from (4.100) and the multiplication formula (3.145).

We proceed by obtaining series and integral representations for the (logarithm of the) $u$-function. From (4.100) and (3.141) we obtain (formally at first)

$$
u(z)=\exp (E(z))=\exp \left(2 i \sum_{n=1}^{\infty} \frac{e^{-b n r} \operatorname{sh}(a-b) n r}{n \operatorname{sh} a n r} \sin 2 n r z\right)
$$

(Alternatively, this can be deduced from (4.87) and (4.92).) The series converges absolutely provided

$$
|\operatorname{Im} z|<d(a, b) / 2
$$

with

$$
d(a, b) \equiv a+b-|a-b|
$$

Thus one has

$$
d(a, b)>a \Leftrightarrow b>a / 2
$$

in agreement with the fact that $u$ is a minimal solution to the $\mathrm{A} \Delta \mathrm{E}$ (4.105) for parameters in $\mathscr{R}$ (4.106). More generally, the series representation (4.114) makes sense and holds true in a strip around the real $z$-axis iff the parameter $b$ is positive.

Next, we take $(r, a, b) \in \mathscr{R}$ and set 


$$
\phi(z) \equiv \ln \left(\frac{\sin r(z-i b+i a / 2) \sin r(z+i b-i a / 2)}{\sin r(z+i a / 2) \sin r(z-i a / 2)}\right)+2 r(a-b)
$$

with $\ln$ real-valued for $z \in \mathbb{R}$. Obviously, $\phi$ satisfies the assumptions $(2.100)$ and $(2.101)$ of Theorem II.5, and comparing (4.105) and (4.114) it follows that $\phi$ satisfies (2.102), too. Thus, (2.107) applies, yielding the integral representation

$$
E(z)=\frac{1}{2 i \pi} \int_{-\pi / 2 r}^{\pi / 2 r} d y \phi(y) K(r, a ; z-y), \quad(r, a, b) \in \mathscr{B}, \quad|\operatorname{Im} z|<\frac{a}{2} .
$$

By continuity, the representation still holds for $b=a / 2$, but it is false in general for $b<a / 2$.

To conclude this subsection, we obtain three limits of the trigonometric scattering function. First, we use (3.155) to write

$$
u(r, 1, b ; z)=\frac{T(r ; z-i b+i / 2) T(r ;-z-i / 2)}{T(r ;-z-i b+i / 2) T(r ; z-i / 2)} \exp (2 i r(b-1) z) .
$$

Then it follows from Prop. III.20 that we have

$$
\lim _{r \downarrow 0} u(r, 1, g ; z)=\frac{\Gamma(-i z+g) \Gamma(i z+1)}{(z \rightarrow-z)} \quad\left(\mathrm{I}_{\mathrm{nr}} \text { limit }\right)
$$

mero-uniformly in $z$. (Compare this to (4.48).)

Second, we observe that

$$
\lim _{a \downarrow 0} u(r, a, a g ; z)=\exp ((1-g)(i \pi-2 i r z)), \quad z \in \mathscr{R}_{r} \quad\left(\hat{\mathrm{III}} \mathrm{n}_{\mathrm{nr}} \text { limit }\right),
$$

uniformly on compact subsets of the period strip $\mathscr{R}_{r}$ (2.113). Indeed, this readily follows from (3.180), cf. also (4.95) and (4.96). The remark below (4.96) applies to the case at hand as well.

Third, we introduce

$$
L_{\hbar}(z) \equiv i \hbar \ln u(r, \hbar / \lambda, b ; z), \quad(r, \lambda, b, z) \in(0, \infty)^{3} \times \mathscr{R}_{r},
$$

with $\ln u \rightarrow 0$ for $z \rightarrow 0$ and $\hbar>0$ Planck's constant. Then we claim that

$$
\lim _{\hbar \rightarrow 0} \partial_{z} L_{\hbar}(z)=\lambda \ln \left(e^{-2 r b} \frac{\sin r(z+i b) \sin r(z-i b)}{\sin ^{2} r z}\right) \quad \text { (classical limit) }
$$

uniformly on compacts of $\mathscr{B}_{r}$, with $\ln$ real-valued for $z \in(0, \pi / r)$. To prove this claim, we use (4.100) and (3.176), (3.177) to write

$$
\begin{aligned}
i a \ln u(r, a, b ; z)= & -D_{a}(z+i b)+D_{a}(-z+i b)-D_{a}(-z)+D_{a}(z)-a d_{a}(r,-1 / 2,0 ; z+i b) \\
& +a d_{a}(r,-1 / 2,0 ;-z+i b)-a d_{a}(r, 1 / 2,0 ;-z)+a d_{a}(r, 1 / 2,0 ; z),
\end{aligned}
$$

where we take $z \in \mathscr{R}_{r}$. Invoking now Prop. III.21, the limit (4.124) readily follows.

Comparing the rhs of (4.124) to the classical phase shift obtained in Ref. 17, p. 336, we get agreement when we take $\lambda \rightarrow \beta^{-1}, r \rightarrow|\mu| / 2, b \rightarrow|\beta g|$, save for a constant shift $-2 \lambda r b \rightarrow-|\mu g|$. The latter shift can be understood from the fact that the distance between the classical actions of the $\mathrm{III}_{\text {rel }}$ system is bounded below by $|\mu \mathrm{g}|$ (cf. Ref. 17, p. 256); by contrast, the minimal distance between successive indices $n_{i}, n_{i+1}$ of the multivariable polynomials occurring at the quantum level equals 0 . (See also Ref. 2, Subsection 6.2.) 


\section{WEIGHT FUNCTIONS}

\section{A. The hyperbolic case}

Our study of the hyperbolic weight function $w\left(a_{+}, a_{-}, b ; z\right)$ runs largely parallel to our study of the $u$-function in Subsection IV A. The $w$-function is defined by

$$
w(z) \equiv \frac{G\left(z+i b-i\left(a_{+}+a_{-}\right) / 2\right) G\left(z+i\left(a_{+}+a_{-}\right) / 2\right)}{G\left(z-i b+i\left(a_{+}+a_{-}\right) / 2\right) G\left(z-i\left(a_{+}+a_{-}\right) / 2\right)},
$$

so it satisfies

$$
w\left(a_{+}, a_{-} ; z\right)=w\left(a_{-}, a_{+} ; z\right)
$$

just as $G(z)$ and $u(z)$, cf. (4.1) and (4.2). The analogs of (4.3) and (4.4) are

$$
\begin{gathered}
w(-z)=w(z), \\
w\left(\lambda a_{+}, \lambda a_{-}, \lambda b ; \lambda z\right)=w\left(a_{+}, a_{-}, b ; z\right), \quad \lambda \in(0, \infty) .
\end{gathered}
$$

For several purposes it is convenient to introduce a reduced weight function

$$
w_{r}(z) \equiv \frac{G\left(z+i b-i\left(a_{+}+a_{-}\right) / 2\right)}{G\left(z-i b+i\left(a_{+}+a_{-}\right) / 2\right)} .
$$

Using the $\mathrm{A} \Delta \mathrm{Es}$ (3.4), one infers that $w$ and $w_{r}$ are related by

$$
w(z)=4 \operatorname{sh}\left(\pi z / a_{+}\right) \operatorname{sh}\left(\pi z / a_{-}\right) w_{r}(z) .
$$

Obviously, $w_{r}$ also satisfies (5.2)-(5.4).

Just as the $u$-function, the functions $w$ and $w_{r}$ are meromorphic in $a_{+}, a_{-}, b$ and $z$, as long as $a_{-} / a_{+}$stays away from $(-\infty, 0]$, cf. Prop. III.5. In particular, both $u$ and $w_{r}$ are well defined for $b, z \in$ C. Using (4.1) and (3.4), one readily verifies that the latter functions are related by

$$
u(i z ; i b)=w_{r}(b ; z) \frac{4 \operatorname{sh} \frac{\pi}{a_{+}}(z+i b) \operatorname{sh} \frac{\pi}{a_{-}}(z+i b)}{G\left(i b-i\left(a_{+}-a_{-}\right) / 2\right) G\left(i b+i\left(a_{+}-a_{-}\right) / 2\right)} .
$$

This relation can be used to translate various features of $w_{r}$ in terms of $u$ and vice versa.

From now on we take $\left(a_{+}, a_{-}, b\right) \in \mathscr{H}(4.5)$. We proceed by studying $w$ and $w_{r}$ with regard to the A $\Delta$ Es they satisfy, namely

$$
\frac{w\left(z+i a_{\delta} / 2\right)}{w\left(z-i a_{\delta} / 2\right)}=\frac{s_{-\delta}\left(z+i b-i a_{\delta} / 2\right)}{s_{-\delta}\left(z-i b+i a_{\delta} / 2\right)} \cdot \frac{s_{-\delta}\left(z+i a_{\delta} / 2\right)}{s_{-\delta}\left(z-i a_{\delta} / 2\right)}
$$

and

$$
\frac{w_{r}\left(z+i a_{\delta} / 2\right)}{w_{r}\left(z-i a_{\delta} / 2\right)}=-\frac{s_{-\delta}\left(z+i b-i a_{\delta} / 2\right)}{s_{-\delta}\left(z-i b+i a_{\delta} / 2\right)},
$$

resp. (To check this, recall the definition (4.7) and the A $\Delta \mathrm{Es}$ (3.4).)

Consider first $w_{r}$. The planes (4.9) separate the region $\mathscr{H}$ (4.5) into infinitely many strip-like components, one of which reads

$$
\mathscr{F}_{\delta} \equiv\left\{\left(a_{+}, a_{-}, b\right) \in \mathscr{H} \mid b \in\left(a_{\delta} / 2, a_{-\delta}+a_{\delta} / 2\right)\right\} .
$$


The pole/zero properties of $G(z)$ given by Prop. III.3 entail that $w_{r}$ is free of zeros and poles in the strip $|\operatorname{Im} z| \leqslant a_{\delta} / 2$ if and only if $\left(a_{+}, a_{-}, b\right) \in \mathscr{S}_{\delta}$. Now from Prop. III.4 we deduce that for all $\left(a_{+}, a_{-}, b\right) \in \mathscr{H}$ one has

$$
w_{r}(z)=\exp \left( \pm \frac{\pi z}{a_{+} a_{-}}\left(2 b-a_{+}-a_{-}\right)\right)\left(1+O\left(\exp \left( \pm\left(\epsilon-2 \pi / a_{m}\right) z\right)\right)\right), \quad \operatorname{Re} z \rightarrow \pm \infty,
$$

uniformly for $\operatorname{Im} z$ in $\mathbb{R}$-compacts. Thus, choosing parameters in $\mathscr{S}_{\delta}$, one may characterize $w_{r}$ as a minimal solution to the $A \Delta \mathrm{E}$ (5.9) that is even and positive for $z \in \mathbb{R}$; these properties determine the solution up to a positive constant, cf. Theorem II.1. Next, we note that the rhs of (5.9) is $a_{-\delta}$-periodic in $b$, and identically equal to -1 for parameters satisfying (4.9). (As such, the $\mathrm{A} \Delta \mathrm{E}$ is regular for all $\left(a_{+}, a_{-}, b\right) \in \mathscr{H}$, by contrast to (4.6).) But $w_{r}$ is neither $a_{-\delta}$-periodic in $b$, nor an exponential when (4.9) holds true. We shall presently obtain the corresponding $i a_{\delta}$-periodic multiplier, after considering $w$ in relation to the $\mathrm{A} \Delta \mathrm{E}(5.8)$ it obeys.

We begin by noting that the $w$-function has asymptotics

$$
w(z)=\exp \left( \pm \frac{2 \pi b z}{a_{+} a_{-}}\right)\left(1+O\left(\exp \left( \pm\left(\epsilon-2 \pi / a_{m}\right) z\right)\right)\right), \quad \operatorname{Re} z \rightarrow \pm \infty .
$$

Thus, it is a minimal solution to (5.8) whenever it has no poles and zeros for $|\operatorname{Im} z| \leqslant a_{\delta} / 2$. In view of (5.6), for this to happen it is necessary that $w_{r}(z)$ have a double pole at $z=0$. For $a_{+}, a_{-}$fixed, this necessary condition is satisfied only for a discrete set of $b$, so $w$ is generically not a regular solution-in contrast to $w_{r}$, which is regular for parameters in $\mathscr{S}_{\delta}$.

It should be pointed out, though, that both of the A $\Delta$ Es (5.8) do admit minimal solutions for all $\left(a_{+}, a_{-}, b\right) \in \mathscr{H}$. (Indeed, this readily follows from Theorem II.3.) In particular, let us introduce the asymmetric weight function

$$
w_{\delta}\left(a_{+}, a_{-}, b ; z\right) \equiv \frac{G\left(z+i b-i\left(a_{+}+a_{-}\right) / 2\right) G\left(z+i\left(a_{\delta}-a_{-\delta}\right) / 2\right)}{G\left(z-i b+i\left(a_{+}+a_{-}\right) / 2\right) G\left(z-i\left(a_{\delta}-a_{-\delta}\right) / 2\right)} .
$$

This function is related to $w_{r}$ and $w$ via

$$
w_{\delta}(z)=w_{r}(z) \frac{\operatorname{sh}\left(\pi z / a_{-\delta}\right)}{\operatorname{sh}\left(\pi z / a_{\delta}\right)}=w(z) / 4 \operatorname{sh}^{2}\left(\pi z / a_{\delta}\right)
$$

on account of (3.5), (5.8) and (5.6). Since $w$ solves (5.8), so does $w_{\delta}$. Choosing the parameters in $\mathscr{R}_{\delta}(4.10), w_{\delta}$ is a minimal solution, as is easily verified. Multiplying and/or dividing $w_{\delta}$ by finitely many factors of the form $s_{\delta}(z-c)$, one can construct explicit minimal solutions for arbitrary parameters.

We continue by obtaining analogs of the formulas (4.13)-(4.20). First, we use the A $\Delta$ Es (3.4) to obtain

$$
\frac{W\left(b+a_{-\delta} ; z\right)}{W(b ; z)}=4 \operatorname{sh} \frac{\pi}{a_{\delta}}(z+i b) \operatorname{sh} \frac{\pi}{a_{\delta}}(z-i b), \quad W=w, w_{r}, w_{+}, w_{-} .
$$

Taking $k_{+}, k_{-} \in \mathbb{Z}$, these $\mathrm{A} \Delta$ Es can be iterated to yield 


$$
\begin{aligned}
& \frac{W\left(b+k_{+} a_{+}+k_{-} a_{-} ; z\right)}{W(b ; z)} \\
& =\prod_{\delta=+,-} \prod_{j_{\delta}=1}^{\left|k_{\delta}\right|}\left(4 \left(\operatorname { s h } \frac { \pi } { a _ { - \delta } | } \left(z+i \frac{k_{\delta}}{\left|k_{\delta}\right|}\left(b-\frac{a_{\delta}}{2}\right)\right.\right.\right. \\
& \left.\left.\left.\quad+i a_{\delta}\left(j_{\delta}-\frac{1}{2}\right)\right)\right)(i \rightarrow-i)\right)^{k_{\delta} /\left|k_{\delta}\right|} .
\end{aligned}
$$

Next, we note that (5.5) and (3.4) entail

$$
\begin{gathered}
w\left(a_{+}, a_{-}, 0 ; z\right)=1, \\
w\left(a_{+}, a_{-}, a_{\delta} / 2 ; z\right)=2 \operatorname{th}\left(\pi z / a_{\delta}\right) \operatorname{sh}\left(\pi z / a_{-\delta}\right), \\
w\left(a_{+}, a_{-},\left(a_{+}+a_{-}\right) / 2 ; z\right)=4 \operatorname{sh}\left(\pi z / a_{+}\right) \operatorname{sh}\left(\pi z / a_{-}\right) .
\end{gathered}
$$

Therefore, the weight functions are elementary functions for parameters in the dense subset

$$
\mathscr{D}_{w} \equiv\left\{\left(a_{+}, a_{-}, b\right) \in \mathscr{H} \mid b=l_{+} a_{+}+l_{-} a_{-}, l_{+}, l_{-} \in \mathbb{Z} / 2\right\}
$$

of $\mathscr{H}$ (4.5). Specifically, one readily obtains from (5.16)-(5.19) (using the notation (4.19) and taking $\left.k_{+}, k_{-} \in \mathbb{Z}\right)$

$$
\begin{aligned}
& w\left(a_{+}, a_{-}, k_{+} a_{+}+k_{-} a_{-} ; z\right)=\prod_{\delta=+,-} \prod_{j_{\delta}=1}^{\left|k_{\delta}\right|}\left(4\left(\operatorname{sh} \frac{\pi}{a_{-\delta}}\left(z+i a_{\delta}\left(j_{\delta}-\theta\left(k_{\delta}\right)\right)\right)\right)(i \rightarrow-i)\right)^{k_{\delta} \prime\left|k_{\delta}\right|}, \\
& w\left(a_{+}, a_{-}, a_{\delta} / 2+k_{+} a_{+}+k_{-} a_{-} ; z\right) \\
& =2 \operatorname{th}\left(\frac{\pi z}{a_{\delta}}\right) \operatorname{sh}\left(\frac{\pi z}{a_{-\delta}}\right) \prod_{j_{\delta}=1}^{\left|k_{\delta}\right|}\left(4\left(\operatorname{sh} \frac{\pi}{a_{-\delta}}\left(z+i a_{\delta}\left(j_{\delta}-\frac{1}{2}\right)\right)\right)(i \rightarrow-i)\right)^{k_{\delta} \prime\left|k_{\delta}\right|} \\
& \cdot \prod_{j=1}^{\left|k_{-\delta}\right|}\left(4\left(\operatorname{ch} \frac{\pi}{a_{\delta}}\left(z+i a_{-\delta}\left(j_{-\delta}-\theta\left(k_{-\delta}\right)\right)\right)\right)(i \rightarrow-i)\right)^{k_{-\delta}|| k_{-\delta} \mid} \text {, } \\
& w\left(a_{+}, a_{-},\left(a_{+}+a_{-}\right) / 2+k_{+} a_{+}+k_{-} a_{-} ; z\right) \\
& =4 \operatorname{sh}\left(\frac{\pi z}{a_{+}}\right) \operatorname{sh}\left(\frac{\pi z}{a_{-}}\right) \prod_{\delta=+,-} \prod_{j_{\delta}=1}^{\left|k_{\delta}\right|}\left(4\left(\operatorname{ch} \frac{\pi}{a_{-\delta}}\left(z+i a_{\delta}\left(j_{\delta}-\frac{1}{2}\right)\right)\right)(i \rightarrow-i)\right)^{k_{\delta}\left|k_{\delta}\right|} .
\end{aligned}
$$

We proceed by noting that none of the weight functions has the reflection symmetry (4.21) of the scattering function. Instead, one gets from (5.5) the relation

$$
w_{r}\left(a_{+}+a_{-}-b ; z\right)=1 / w_{r}(b ; z) .
$$

Combining this with (5.16), one obtains

$$
w_{r}(-b ; z) w_{r}(b ; z)=\prod_{\delta=+,-}\left(4 \operatorname{sh} \frac{\pi}{a_{\delta}}(z+i b) \operatorname{sh} \frac{\pi}{a_{\delta}}(z-i b)\right)^{-1} .
$$


Using the multiplication formula (3.25), one can work out analogs of the relations (4.23)(4.26) for the weight functions. We shall not do so, however. We do point out that $w_{r}$ satisfies an additional relation involving shifts of $b$-as opposed to shifts of $z$ :

$$
w_{r}\left(\frac{a_{+}}{M}, \frac{a_{-}}{N}, b ; z\right)=\prod_{j=0}^{M-1} \prod_{k=0}^{N-1} w_{r}\left(a_{+}, a_{-}, b+\frac{a_{+}}{M} j+\frac{a_{-}}{N} k ; z\right) .
$$

(Indeed, this formula readily follows from (5.5) and (3.25).)

By contrast to the scattering function, the weight functions are elementary functions on all of the sine-Gordon lines. In particular, from (5.6) and (5.18) we have

$$
w(\pi, \alpha, \pi / 2 ; z)=2 \operatorname{th} z \operatorname{sh}\left(\pi \alpha^{-1} z\right) \quad(\mathrm{sG})
$$

for all $\alpha>0$. (Compare this to (4.28).)

Next, we obtain an integral representation for $w_{r}$ : From (3.1), (3.3) and (5.5) we have

$$
w_{r}(z)=\exp (I(z))
$$

where

$$
I(z) \equiv \int_{0}^{\infty} \frac{d y}{y}\left(\frac{\operatorname{sh}\left(a_{+}+a_{-}-2 b\right) y}{\operatorname{sh} a_{+} y \operatorname{sh} a_{-} y} \cos 2 y z-\frac{a_{+}+a_{-}-2 b}{a_{+} a_{-} y}\right) .
$$

This integral converges absolutely provided

$$
|\operatorname{Im} z|<e\left(a_{+}, a_{-}, b\right) / 2
$$

where

$$
e\left(a_{+}, a_{-}, b\right) \equiv a_{+}+a_{-}-\left|2 b-a_{+}-a_{-}\right| .
$$

Thus we have in particular

$$
e\left(a_{+}, a_{-}, b\right)>a_{\delta} \Leftrightarrow\left(a_{+}, a_{-}, b\right) \in \mathscr{S}_{\delta},
$$

which says once more that $w_{r}$ is regular for parameters in $\mathscr{S}_{\delta}$.

More generally, the integral representation (5.28) sense and holds true in a strip around the real $z$-axis iff the parameters belong to $\mathscr{C}$ (4.34). Indeed, one clearly has

$$
e\left(a_{+}, a_{-}, b\right)>0 \Leftrightarrow\left(a_{+}, a_{-}, b\right) \in \mathscr{C} \text {. }
$$

Combining the representation with (5.6), (5.14) and (5.15), we obtain the positivity property

$$
W\left(a_{+}, a_{-}, b ; x\right)>0, \quad \forall\left(a_{+}, a_{-}, b, x\right) \in \mathscr{H} \times \mathbb{R}^{*}, \quad W=w, w_{r}, w_{+}, w_{-} .
$$

From (3.1) and (3.3) we also obtain an integral representation for the asymmetric weight function $w_{\delta}(5.13)$, viz.,

$$
w_{\delta}(z)=\exp \left(I_{\delta}(z)\right)
$$

with

$$
I_{\delta}(z) \equiv 2 \int_{0}^{\infty} \frac{d y}{y}\left(\frac{\operatorname{sh}\left(a_{-\delta}-b\right) y \operatorname{ch}\left(a_{\delta}-b\right) y}{\operatorname{sh} a_{+} y \operatorname{sh} a_{-} y} \cos 2 y z-\frac{a_{-\delta}-b}{a_{+} a_{-} y}\right) .
$$


Obviously, this integral has the same convergence properties as the integral (4.30), so the analysis embodied in (4.31)-(4.35) applies once again.

We have not found illuminating analogs of the representations (4.38) and (4.45), so we conclude this subsection by deriving two limits of the weight function $w$. (Corresponding limits for $w_{r}, w_{+}$and $w_{-}$readily follow, so they will not be spelled out.) Once again, we switch to parameters (4.47).

First, we use (5.1), (3.22), (3.24) and (3.69) to obtain

$$
w(\pi, \beta \nu, \beta \nu g ; \beta p)=\exp (2 g \ln (2 \beta \nu)) \frac{H(\rho ; p / \nu+i / 2) H(\rho ;-p / \nu+i / 2)}{H(\rho ; p / \nu-i g+i / 2) H(\rho ;-p / \nu-i g+i / 2)}, \quad \rho \equiv \beta \nu / \pi .
$$

Therefore, Prop. III.6 entails

$$
\lim _{\beta \downarrow 0}(2 \beta \nu)^{-2 g} w(\pi, \beta \nu, \beta \nu g ; \beta p)=\frac{\Gamma(i p / \nu+g) \Gamma(-i p / \nu+g)}{\Gamma(i p / \nu) \Gamma(-i p / \nu)} \quad\left(\mathrm{I}_{\mathrm{rel}} \text { limit }\right),
$$

where the limit is mero-uniform. (The limiting weight function is associated to the analytic difference operators of the $I_{\text {rel }}$ regime, cf. Refs. 1 and 2.)

Second, we may write

$$
w(\pi, a, a g ; z)=\frac{G(\pi, a ; z-i \pi / 2+i a(g-1 / 2))}{G(\pi, a ; z-i \pi / 2+i a(-1 / 2))} \cdot \frac{G(\pi, a ; z+i \pi / 2+i a(1 / 2))}{G(\pi, a ; z+i \pi / 2+i a(1 / 2-g))} .
$$

Therefore, we deduce from (3.91)

$$
\lim _{\beta \downarrow 0} w(\pi, \beta \nu, \beta \nu g ; \nu x)=\exp (2 g \ln (2 \operatorname{sh} \nu x)), \quad x \in \mathscr{\cap}_{0} \quad\left(\mathrm{II}_{\mathrm{nr}} \text { limit }\right)
$$

(with In real-valued for $x>0$ ), uniformly on compacts of $\varkappa_{0}$. (The limit is the weight function of the $\mathrm{II}_{\mathrm{nr}}$ regime, cf. Refs. 1 and 2)

\section{B. The elliptic case}

The elliptic $w$-function is defined by replacing in (5.1) the hyperbolic $G$-functions by their elliptic counterparts. Obviously, this yields a function that is periodic in $z$. with primitive period $\pi / r$, and which satisfies (5.2), (5.3), and (4.76), (4.77) with $u$ replaced by $w$.

Just as in the hyperbolic case, we introduce a reduced weight function by (5.5). Then we obtain via (3.100) and (3.96)-(3.99)

$$
w(z)=4 r^{2} \prod_{k=1}^{\infty}\left(1-q_{+}^{2 k}\right)^{2}\left(1-q_{-}^{2 k}\right)^{2} \cdot s_{+}(z) s_{-}(z) w_{r}(z) .
$$

Evidently, $w_{r}$ shares the automorphy properties of $w$ mentioned above.

From Prop. III. 11 we deduce that $w$ and $w_{r}$ are meromorphic in $r, a_{+}, a_{-}, b$ and $z$, provided $a_{+} r$ and $a_{-} r$ stay in the right half plane. As the analog of (5.7) we then obtain

$$
u(i z ; i b)=w_{r}(b ; z) \frac{4 r^{2} \Pi_{k=1}^{\infty}\left(1-q_{+}^{2 k}\right)^{2}\left(1-q_{-}^{2 k}\right)^{2} \cdot s_{+}(z+i b) s_{-}(z+i b)}{G\left(i b-i\left(a_{+}-a_{-}\right) / 2\right) G\left(i b+i\left(a_{+}-a_{-}\right) / 2\right)} .
$$

From now on we take the parameters in $\zeta(4.75)$. Turning to the A Es satisfied by $w$ and $w_{r}$, we obtain once more 


$$
\frac{w\left(z+i a_{\delta} / 2\right)}{w\left(z-i a_{\delta} / 2\right)}=\frac{s_{-\delta}\left(z+i b-i a_{\delta} / 2\right)}{s_{-\delta}\left(z-i b+i a_{\delta} / 2\right)} \cdot \frac{s_{-\delta}\left(z+i a_{\delta} / 2\right)}{s_{-\delta}\left(z-i a_{\delta} / 2\right)},
$$

whereas (5.9) is replaced by

$$
\frac{w_{r}\left(z+i a_{\delta} / 2\right)}{w_{r}\left(z-i a_{\delta} / 2\right)}=-\exp (2 i r z) \frac{s_{-\delta}\left(z+i b-i a_{\delta} / 2\right)}{s_{-\delta}\left(z-i b+i a_{\delta} / 2\right)}
$$

Considering first $w_{r}$, we reach the same conclusion as in the hyperbolic case-Prop. III.10 and $\pi / r$-periodicity in $z$ play the role of Prop. III.3 and the asymptotics (5.11). Turning to $w(z)$, one readily sees that it generically has double zeros at $z=k \pi / r, k \in \mathbb{Z}$, and hence is not regular. The asymmetric function $w_{\delta}$ defined by (5.13) is now related to $w_{r}$ and $w$ via

$$
w_{\delta}(z)=w_{r}(z) \prod_{k=1}^{\infty}\left(\frac{1-q_{-\delta}^{2 k}}{1-q_{\delta}^{2 k}}\right)^{2} \cdot \frac{s_{-\delta}(z)}{s_{\delta}(z)}=\frac{w(z)}{4 r^{2} \Pi_{k=1}^{\infty}\left(1-q_{\delta}^{2 k}\right)^{4} \cdot s_{\delta}(z)^{2}} .
$$

Since $s_{\delta}(z)^{2}$ is not $i a_{\delta}$-periodic, $w_{\delta}$ does not satisfy the $\mathrm{A} \Delta \mathrm{E}$ (5.43), however. To obtain minimal periodic solutions to (5.43), one should rather multiply $w(z)$ by an elliptic function with periods $\pi / r$ and $i a_{\delta}$. We shall neither embark on this nor on a study of the A $\Delta$ Es solved by the functions $w_{+}$and $w_{-}$.

We continue by obtaining the counterparts of (5.15)-(5.19). First, from (5.1), (5.45) and (3.100) we readily get

$$
\frac{W\left(b+a_{-\delta} ; z\right)}{W(b ; z)}=4 r^{2} e^{-2 r b} \prod_{k=1}^{\infty}\left(1-q_{\delta}^{2 k}\right)^{4} \cdot s_{\delta}(z+i b) s_{\delta}(z-i b), \quad W=w, w_{r}, w_{+}, w_{-} .
$$

To obtain the analog of (5.16), we employ the relation

$$
s\left(r, a ; z_{+}+i n a\right) s\left(r, a ; z_{-}-i n a\right)=e^{-2 i r n\left(z_{+}-z_{-}\right)} e^{2 a r n^{2}} s\left(r, a ; z_{+}\right) s\left(r, a ; z_{-}\right), \quad n \in \mathbb{N},
$$

which is easily derived from (2.91). (This formula plays the same role as (4.80) in simplifying the iterated A $\Delta$ Es.) A straightforward calculation now yields (with $k_{+}, k_{-} \in \mathbb{Z}$ )

$$
\begin{aligned}
\frac{W\left(b+k_{+} a_{+}+k_{-} a_{-} ; z\right)}{W(b ; z)}= & \exp \left(2 r b\left(2 k_{+} k_{-}-k_{+}-k_{-}\right)\right) \prod_{\delta=+,-} \exp \left(r a _ { \delta } k _ { \delta } ( k _ { \delta } - 1 ) \left(2 k_{-\delta}\right.\right. \\
& -1)) \prod_{j_{\delta}=1}^{\left|k_{\delta}\right|}\left(4 r ^ { 2 } \prod _ { k = 1 } ^ { \infty } ( 1 - q _ { - \delta } ^ { 2 k ^ { 2 } } ) ^ { 4 } \left(s _ { - \delta } \left(z+i \frac{k_{\delta}}{\left|k_{\delta}\right|}\left(b-\frac{a_{\delta}}{2}\right)\right.\right.\right. \\
& \left.\left.\left.+i a_{\delta}\left(j_{\delta}-\frac{1}{2}\right)\right)\right)(i \rightarrow-i)\right)^{k_{\delta} /\left|k_{\delta}\right|} .
\end{aligned}
$$

Next, we use (5.1) and (3.100) to obtain

$$
\begin{gathered}
w\left(r, a_{+}, a_{-}, 0 ; z\right)=1, \\
w\left(r, a_{+}, a_{-}, a_{\delta} / 2 ; z\right)=4 r^{2} \prod_{k=1}^{\infty}\left(1-q_{+}^{2 k}\right)^{2}\left(1-q_{-}^{2 k}\right)^{2} \cdot \frac{s_{\delta}(z)}{R_{\delta}(z)} s_{-\delta}(z),
\end{gathered}
$$




$$
w\left(r, a_{+}, a_{-},\left(a_{+}+a_{-}\right) / 2 ; z\right)=4 r^{2} \prod_{\delta=+,-} \prod_{k=1}^{\infty}\left(1-q_{\delta}^{2 k}\right)^{2} \cdot s_{\delta}(z) .
$$

If we now combine these formulas with the quotient formula (5.48), we obtain obvious analogs of (5.21)-(5.23) - which we do not spell out.

We proceed by observing that (5.24) holds true for the elliptic $w_{r}$, too. In tandem with (5.48), this entails

$$
w_{r}(-b ; z) w_{r}(b ; z)=\prod_{\delta=+,-}\left(4 r^{2} \prod_{k=1}^{\infty}\left(1-q_{\delta}^{2 k}\right)^{4} \cdot s_{\delta}(z+i b) s_{\delta}(z-i b)\right)^{-1} .
$$

Analogs of (4.23)-(4.26) for the elliptic weight functions are readily derived from the multiplication formula (3.25), so they will be skipped. The latter formula also entails that the elliptic $w_{r}$-function obeys (5.26).

As the elliptic counterpart of (5.27) we obtain from (5.50) and (5.41)

$$
w(r, \pi, \alpha, \pi / 2 ; z)=4 r^{2} \prod_{k=1}^{\infty}\left(1-e^{-2 k \pi r}\right)^{2}\left(1-e^{-2 k \alpha r}\right)^{2} \cdot \frac{s(r, \pi ; z)}{R(r, \pi ; z)} s(r, \alpha ; z) \quad(\mathrm{XYZ}) .
$$

This holds true for all $\alpha>0$, as opposed to the explicit formula (4.86), which holds for the dense set (4.27).

We now turn to deriving and studying a series representation for $w_{r}$. Recalling (3.3) and (3.92), the definition (5.5) entails

$$
w_{r}(z)=\exp (S(z))=\exp \left(\sum_{n=1}^{\infty} \frac{\operatorname{sh}\left(a_{+}+a_{-}-2 b\right) n r}{n \operatorname{sh} a_{+} n r \operatorname{sh} a_{-} n r} \cos 2 n r z\right) .
$$

The convergence properties of the infinite series $S(z)$ occurring here are the same as those of the integral $I(z)$ (5.29), so the analysis encoded in (5.30)-(5.33) applies verbatim. Using this representation, (5.46) and (5.45), we now deduce the positivity property

$$
W\left(r, a_{+}, a_{-}, b ; x\right)>0, \quad \forall\left(r, a_{+}, a_{-}, b, x\right) \in \mathscr{E} \times(0, \pi / r), \quad W=w, w_{r}, w_{+}, w_{-} .
$$

It is of interest to compare the series representation (5.54) to Theorem II.5. Choosing parameters in $\mathscr{Y}_{\delta}$, one deduces that Theorem II.5 applies to the additive version of (5.44), and that $w_{r}$ corresponds to the unique minimal solution (2.106). Via (2.107) one can now obtain an integral representation for $w_{r}$-as an analog of the representation (4.89) for the elliptic $u$-function.

To conclude this subsection, we derive three limits of the $w$-function. First, we use Prop. III.12 to infer

$$
\lim _{r \downarrow 0} \exp \left(\frac{\pi^{2} b}{3 r a_{+} a_{-}}\right) w\left(r, a_{+}, a_{-}, b ; z\right)=w_{\text {hyp }}\left(a_{+}, a_{-}, b ; z\right) \quad\left(\mathrm{II}_{\mathrm{rel}} \text { limit }\right),
$$

where the limit is mero-uniform. (Here, $w_{\text {hyp }}$ denotes the $w$-function from Subsection V A.) Note that the renormalizing exponential is necessary, and that no such factor occurs in the $u$-function counterpart (4.91).

Next, we claim that the limit

$$
\lim _{A \uparrow \infty} w(r, a, A, b ; z)=w_{\text {trig }}(r, a, b ; z) \quad\left(\mathrm{III}_{\mathrm{rel}} \text { limit }\right)
$$


exists mero-uniformly. (Here, $w_{\text {trig }}$ denotes the $w$-function studied in the next subsection.) Indeed, we may rewrite (5.1) as

$$
w(r, a, A, b ; z)=\frac{G(r, a, A ; z+i b-i a / 2-i A / 2) G(r, a, A ;-z+i b-i a / 2-i A / 2)}{G(r, a, A ; z-i a / 2-i A / 2) G(r, a, A ;-z-i a / 2-i A / 2)},
$$

so (3.139) yields the mero-uniform limit

$$
\lim _{A \uparrow \infty} w(r, a, A, b ; z)=\frac{G(r, a ; z+i b-i a / 2) G(r, a ;-z+i b-i a / 2)}{G(r, a ; z-i a / 2) G(r, a ;-z-i a / 2)} .
$$

In view of (5.61) below, this entails (5.57).

Finally, fixing $g \in \mathbb{R}$, one has

$$
\lim _{a \downarrow 0} w(r, A, a, a g ; z)=\exp \left(2 g \ln \left(2 r \prod_{k=1}^{\infty}\left(1-e^{-2 k A r}\right)^{2} \cdot s(r, A ; z)\right)\right), \quad z \in \mathscr{B}_{r} \quad\left(\mathrm{IV}_{\mathrm{nr}} \text { limit }\right)
$$

(with ln real for $z \in(0, \pi / r)$ ), uniformly on compacts of $\mathscr{B}_{r}(2.113)$. To check this, one need only substitute $b=a g$ in (5.58), invoke the limit (3.138), and recall (3.96)-(3.99).

\section{The trigonometric case}

The trigonometric $w$-function is defined by

$$
w(r, a, b ; z) \equiv \frac{G(z+i b-i a / 2) G(-z+i b-i a / 2)}{G(z-i a / 2) G(-z-i a / 2)}
$$

with $G$ given by (3.140). Thus, it can be written

$$
w(r, a, b ; z)=\prod_{n=0}^{\infty}\left(\frac{1-q^{2 n} e^{2 i r z}}{1-q^{2 n} e^{-2 r b+2 i r z}}\right)(z \rightarrow-z), \quad q \equiv e^{-a r} .
$$

We note that $w$ is $\pi / r$-periodic and even in $z$, and satisfies (4.103) and (4.104) with $u$ replaced by $w$.

Next, we introduce the reduced weight function

$$
w_{r}(z) \equiv G(z+i b-i a / 2) G(-z+i b-i a / 2),
$$

which has the same automorphy properties as $w$. Recalling the functional equation (3.154) and $\mathrm{A} \triangle \mathrm{E}$ (3.142) satisfied by the trigonometric $G$-function, one readily verifies that $w_{r}$ and $w$ are related by

$$
w(z)=4 r \prod_{l=1}^{\infty}\left(1-q^{2 l}\right)^{2} \cdot s(r, a ; z) \sin (r z) w_{r}(z)
$$

Obviously, $w_{r}$ and $w$ are meromorphic in $r, a, b$ and $z$, as long as $a r$ stays in the right half plane. As the counterpart of (5.42) one easily gets

$$
u(i z ; i b)=4 r \prod_{l=1}^{\infty}\left(1-q^{2 l}\right)^{2} \cdot s(r, a ; z+i b) \sin r(z+i b) \frac{G(-i b+i a / 2)}{G(i b+i a / 2)} w_{r}(b ; z) .
$$


Taking from now on parameters in $\mathscr{T}(4.102)$, we turn to the A $\Delta$ Es solved by $w$ and $w_{r}$, viz.,

$$
\frac{w(z+i a / 2)}{w(z-i a / 2)}=\frac{\sin r(z+i b-i a / 2)}{\sin r(z-i b+i a / 2)} \cdot \frac{\sin r(z+i a / 2)}{\sin r(z-i a / 2)}
$$

and

$$
\frac{w_{r}(z+i a / 2)}{w_{r}(z-i a / 2)}=-\exp (2 i r z) \frac{\sin r(z+i b-i a / 2)}{\sin r(z-i b+i a / 2)}
$$

Clearly, both $\mathrm{A} \Delta \mathrm{Es}$ are regular for arbitrary parameters. Choosing parameters in $\mathscr{R}$ (4.106), one readily verifies that the reduced weight function is a minimal solution to (5.67) that is even and positive for $z \in \mathbb{R}$. As such, it is uniquely determined up to a positive constant, cf. Theorem II.1. For $b \leqslant a / 2$, however, it has poles in the strip $|\operatorname{Im} z| \leqslant a / 2$, so it is not regular. The weight function $w(z)$ has double zeros for $z=k \pi / r, k \in \mathbb{Z}$, unless $b=-n a, n \in \mathbb{N}$; in the latter case one easily sees that $w$ is a minimal solution to (5.66).

To proceed, we note that $w$ and $w_{r}$ satisfy the $b-\mathrm{A} \Delta \mathrm{E}$

$$
\frac{W(b+a ; z)}{W(b ; z)}=4 e^{-2 r b} \sin r(z+i b) \sin r(z-i b), \quad W=w, w_{r}
$$

Hence, iteration yields (with $k \in \mathbb{Z}$ )

$$
\frac{W(b+k a ; z)}{W(b ; z)}=e^{-2 r b k-\operatorname{ark}(k-1)} \prod_{j=1}^{|k|}\left(4\left(\sin r\left(z+i \frac{k}{|k|}\left(b-\frac{a}{2}\right)+i a\left(j-\frac{1}{2}\right)\right)\right)(i \rightarrow-i)\right)^{k /|k|} .
$$

Now from (5.61) we read off

$$
w(r, a, 0 ; z)=1,
$$

so we deduce

$$
w(r, a, k a ; z)=e^{-\operatorname{ark}(k-1)} \prod_{j=1}^{|k|}(4[\sin r(z+i a(j-\theta(k)))][i \rightarrow-i])^{k /|k|},
$$

where $k \in \mathbb{Z}$ and the notation (4.19) is used. Moreover, from (3.154) we have

$$
w_{r}(r, a, a / 2 ; z)=R(r, a ; z)^{-1},
$$

so recalling (5.64) we obtain (with $k \in \mathbb{Z}$ )

$$
\begin{aligned}
w(r, a, a / 2+k a ; z)= & 4 r \prod_{l=1}^{\infty}\left(1-q^{2 l}\right)^{2} \cdot \frac{s(r, a ; z)}{R(r, a ; z)} \sin r z \\
& \cdot \prod_{j=1}^{|k|}\left(4\left(\sin r\left(z+i a\left(j-\frac{1}{2}\right)\right)\right)(i \rightarrow-i)\right)^{k /|k|} .
\end{aligned}
$$

Using the multiplication formula (3.145), one easily derives analogs of (4.112) and (4.113) for the weight functions. In addition, (3.145) entails that $w_{r}$ satisfies 


$$
w_{r}\left(r, \frac{a}{M}, b ; z\right)=\prod_{k=0}^{M-1} w_{r}\left(r, a, b+\frac{a}{M} k ; z\right) .
$$

Next, we use (3.141) to obtain a series representation for $w_{r}$, namely

$$
w_{r}(z)=\exp \left(\sum_{n=1}^{\infty} \frac{e^{n r(a-2 b)}}{n \operatorname{sh} n r a} \cos 2 n r z\right) .
$$

Provided $b>0$, this representation makes sense and holds true for $|\operatorname{Im} z|<b$. In particular, this entails once more that $w_{r}$ is a minimal solution to (5.67) when the parameters belong to $\mathscr{R}$ (4.106). (More specifically, $w_{r}$ amounts to the unique minimal solution given by (2.106).) Furthermore, using (5.68) and (5.64) one deduces

$$
W(r, a, b ; x)>0, \quad \forall(r, a, b, x) \in \mathscr{T} \times(0, \pi / r), \quad W=w, w_{r} .
$$

We finish this subsection by obtaining two limits of the trigonometric weight function $w$. Recalling (3.155), we rewrite (5.61) with $a=1$ as

$$
w(r, 1, b ; z)=\frac{T(r ;-z+i / 2) T(r ; z+i / 2)}{T(r ;-z-i b+i / 2) T(r ; z-i b+i / 2)} \exp (r b(1-b)+2 b \ln (2 r)) .
$$

From Prop. III.20 we now infer

$$
\lim _{r \downarrow 0}(2 r)^{-2 g} w(r, 1, g ; z)=\frac{\Gamma(-i z+g) \Gamma(i z+g)}{\Gamma(-i z) \Gamma(i z)} \quad\left(\mathrm{I}_{\mathrm{rel}} \operatorname{limit}\right),
$$

where the limit is mero-uniform. (Compare this to (5.38).)

Next, we substitute $b=a g$, with $g \in \mathbb{R}$ fixed, in (5.61). Recalling then the limit (3.180), we deduce

$$
\lim _{a \downarrow 0} w(r, a, a g ; z)=\exp (2 g \ln (2 \sin r z)), \quad z \in \mathscr{B}_{r} \quad\left(\mathrm{III}_{\mathrm{nr}} \quad \text { limit }\right)
$$

(with $\ln$ real-valued for $z \in(0, \pi / r)$ ), where the limit is uniform on compact subsets of the period strip $\mathscr{R}_{r}(2.113)$.

\section{ACKNOWLEDGMENT}

This work was partially supported by the Netherlands Organisation for the Advancement of Research (NWO).

\section{APPENDIX A: THE GAMMA FUNCTION AND RELATED INTEGRALS}

This appendix serves a twofold purpose. First of all, it is included to render this paper more self-contained. Indeed, most of the Laplace, sine and cosine transforms we derive below can be found-without proof-in standard sources such as Refs. 18 and 19; moreover, all of the properties of the psi and gamma functions we need can be found-with detailed proofs-in various sources, for instance Ref. 16. Our second purpose, however, is to demonstrate how these properties can be very quickly derived via the minimal solution (2.26) to a suitable $\mathrm{A} \Delta \mathrm{E}$ (2.22); this yields a paradigm for the study of generalized psi and gamma functions undertaken in Section III.

Specifically, our starting point is the $\mathrm{A} \Delta \mathrm{E}$ 


$$
F(z+i / 2)-F(z-i / 2)=\frac{i}{z-i / 2} \equiv \chi(z)
$$

A contour integration yields

$$
\hat{\chi}(y)=\frac{1}{2 \pi} \int_{-\infty}^{\infty} d x \frac{i}{x-i / 2} e^{i x y}=-e^{-y / 2} \theta(y),
$$

so this $A \Delta E$ is of the type considered in the proof of Theorem II.3. Indeed, (A2) entails

$$
\hat{\phi}(y)=i y e^{-y / 2} \theta(y), \quad \phi(z) \equiv \chi^{\prime}(z)=-i(z-i / 2)^{-2},
$$

and therefore $\phi(z)$ has all of the properties (2.18)-(2.21). From Theorem II.2 we now obtain a solution

$$
f(z)=2 i \int_{0}^{\infty} d y \frac{y e^{-y}}{\operatorname{sh} y} e^{-2 i y z}, \quad \operatorname{Im} z<1
$$

to the $\mathrm{A} \Delta \mathrm{E}$ (2.22), which is the uniquely determined solution with properties (2.23)-(2.25).

As a consequence, the function

$$
F_{1}(z)=F_{1}(0)+c_{1} z+\int_{0}^{\infty} d y \frac{e^{-y}}{\operatorname{shy}}\left(1-e^{-2 i y z}\right)
$$

is a solution to (A1) for a certain $c_{1} \in \mathrm{C}$. Now we have

$$
F_{1}(i / 2)-F_{1}(-i / 2)=i c_{1}+\int_{0}^{\infty} d y \frac{e^{-y}}{\operatorname{sh} y}\left(-e^{y}+e^{-y}\right)=i c_{1}-2 .
$$

Hence, noting $\chi(0)=-2$, we need $c_{1}=0$ to solve (A1). Of course, we are free to choose $F_{1}(0)$, and we shall set

$$
F_{1}(0)=\int_{0}^{\infty} d y\left(\frac{e^{-2 y}}{y}-\frac{e^{-y}}{\text { shy }}\right) \equiv-\gamma
$$

(As will soon become clear, $\gamma$ is Euler's constant.) The upshot is that we obtain a solution

$$
F_{1}(z) \equiv \int_{0}^{\infty} d y\left(\frac{e^{-2 y}}{y}-\frac{e^{-y(1+2 i z)}}{\operatorname{sh} y}\right), \quad \operatorname{Im} z<1,
$$

to the $\mathrm{A} \Delta \mathrm{E}(\mathrm{A} 1)$. Note that the function $F_{2}(z) \equiv F_{1}(-z+i)$ yields a second solution to (A1), so that $F_{1}(z)-F_{1}(-z+i)$ is an $i$-periodic meromorphic function (determined explicitly below).

Next, we observe that the $\mathrm{A} \Delta \mathrm{E}(2.22)$, with $\phi(z)$ given by (A3), can also be solved by downward iteration, yielding the solution

$$
\widetilde{f}(z)=-i \sum_{k=1}^{\infty}(z-i k)^{-2}
$$

Now this solution clearly has the properties $(2.23)-(2.25)$, so we must have $\widetilde{f}(z)=f(z)$. From this we readily deduce 


$$
F_{1}(z)=-\gamma+i \sum_{k=1}^{\infty}\left(\frac{1}{z-i k}+\frac{1}{i k}\right)
$$

(Indeed, the function on the rhs has derivative $\widetilde{f}(z)=f(z)$ and value $-\gamma$ for $z=0$, just as $F_{1}(z)$, (A7) and (A8).) As a consequence, we obtain the functional equation

$$
F_{1}(z+i / 2)-F_{1}(-z+i / 2)=-\sum_{n=0}^{\infty}\left(\frac{1}{i z+n+1 / 2}+\frac{1}{i z-n-1 / 2}\right)=i \pi \operatorname{th} \pi z .
$$

Note that the rhs amounts to the $i$-periodic meromorphic function mentioned below (A8).

We are now prepared to make contact with the psi and gamma functions. First, we introduce

$$
\psi(z) \equiv F_{1}(-i z+i)=\int_{0}^{\infty} d y\left(\frac{e^{-2 y}}{y}-\frac{e^{y(1-2 z)}}{\operatorname{sh} y}\right), \quad \operatorname{Re} z>0 .
$$

Then we obtain from (A1) and (A11) the $\mathrm{A} \Delta \mathrm{E}$

$$
\psi(z+1)-\psi(z)=1 / z
$$

and functional equation

$$
\psi(z+1 / 2)-\psi(-z+1 / 2)=\pi \operatorname{tg} \pi z
$$

Moreover, we have $\psi(1)=-\gamma$ and $\psi(z)$ has simple poles at $z=0,-1,-2, \ldots$, cf. (A10).

Next, consider any primitive $\Psi(z)$ of $\psi(z)$, restricted to the cut plane

$$
\mathrm{C}^{-} \equiv\{z \in \mathbb{C} \mid z \notin(-\infty, 0]\} .
$$

Clearly, $\Psi(z)$ is analytic in $\mathrm{C}^{-}$and satisfies

$$
\begin{gathered}
\Psi(z+1)-\Psi(z)=\ln z+c_{1}, \quad z \in \mathbb{C}^{-}, \\
\Psi(z+1 / 2)+\Psi(-z+1 / 2)=-\ln (\cos \pi z)+c_{2}, \quad \pm z \notin[1 / 2, \infty),
\end{gathered}
$$

in view of (A13) and (A14). Now from (A12) we have

$$
\Psi(2)-\Psi(1)=\int_{1}^{2} d w \psi(w)=\int_{0}^{\infty} d y\left(\frac{e^{-2 y}}{y}+\frac{e^{y}}{2 y \operatorname{sh} y}\left(e^{-4 y}-e^{-2 y}\right)\right)=0,
$$

so that $c_{1}=0$ in (A16). Clearly, $c_{2}$ in (A17) depends on the arbitrary constant in $\Psi(z)$; we render $\Psi$ unique by requiring $2 \Psi(1 / 2)=\ln \pi$ and then we get $c_{2}=\ln \pi$ by taking $z=0$ in (A17).

The upshot is that we obtain a primitive $\Psi(z)$ of $\psi(z)$ satisfying

$$
\begin{gathered}
\Psi(z+1)-\Psi(z)=\ln z \\
\Psi(z+1 / 2)+\Psi(-z+1 / 2)=\ln (\pi / \cos \pi z) .
\end{gathered}
$$

Introducing the function

$$
\Gamma(z) \equiv \exp (\Psi(z))
$$


(defined at first in $\mathrm{C}^{-}$), it readily follows that $\Gamma(z)$ extends to a meromorphic function without zeros and with simple poles at $z=0,-1,-2, \ldots$. Indeed, from (A10) and (A12) we deduce that we have

$$
\Psi(z)=\alpha-\gamma z-\ln z-\sum_{n=1}^{\infty}\left(\ln \left(1+\frac{z}{n}\right)-\frac{z}{n}\right)
$$

for some $\alpha \in \mathbb{C}$ (with $\ln z$ real for $z>0$, of course). Therefore, we obtain

$$
\frac{1}{\Gamma(z)}=e^{-\alpha+\gamma z} z \prod_{n=1}^{\infty}\left(1+\frac{z}{n}\right) e^{-z / n}
$$

and from this the assertion is clear. From (A19) and (A20) we also obtain the $\mathrm{A} \Delta \mathrm{E}$

$$
\Gamma(1+z)=z \Gamma(z)
$$

and functional equation

$$
\Gamma(z+1 / 2) \Gamma(-z+1 / 2)=\pi / \cos \pi z
$$

In order to determine $\alpha$, we note that (A23) and (A24) entail

$$
e^{-\alpha}=\lim _{z \rightarrow 0} \frac{1}{z \Gamma(z)}=\frac{1}{\Gamma(1)}
$$

Now from (A24) and (A25) we have

$$
\Gamma(z+1 / 2) \Gamma(-z+3 / 2)=\frac{\pi(-z+1 / 2)}{\cos \pi z},
$$

which yields $\Gamma(1)^{2}=1$ for $z \rightarrow 1 / 2$. Thus we conclude

$$
\Gamma(1)=1, \quad \alpha=0,
$$

since $\Gamma(z)$ is positive for $z>0$. (To see this, note that (A12) entails $\psi(z)$ is real for $z>0$. As $\Psi(1 / 2)$ is real, it follows that $\Psi(z)$ is real for $z>0$, so positivity is clear from (A21).)

Combining (A23) and (A28), we see that $\Gamma(z)$ is the customary gamma function in Weierstrass product form, as anticipated by our notation. Similarly, $\psi(z)$ is the usual psi function (the logarithmic derivative of the gamma function), and (A12) amounts to Gauss' formula, cf., e.g., Ref. 16.

We now derive a number of definite integrals by exploiting the properties of $\psi(z)$ and $\Gamma(z)$ established above. The order in which this is done is determined by the order in which these integrals are needed in the main text, except when logical necessity requires otherwise.

First, we use the well-known integral

$$
\int_{0}^{\infty} \frac{d y}{y}\left(e^{-q y}-e^{-p y}\right)=\int_{0}^{\infty} d y \int_{q}^{p} d s e^{-s y}=\int_{q}^{p} \frac{d s}{s}=\ln (p / q)
$$

and (A12) to obtain

$$
\psi(z+1 / 2)-\ln z=\int_{0}^{\infty} d y\left(\frac{1}{y}-\frac{1}{\operatorname{sh} y}\right) e^{-2 y z}, \quad \operatorname{Re} z>0 .
$$


Integrating this from 0 to $z$ and using $2 \Psi(1 / 2)=\ln \pi$, we arrive at

$$
\Psi\left(z+\frac{1}{2}\right)-\frac{1}{2} \ln \pi-z \ln z+z=\frac{1}{2} \int_{0}^{\infty} \frac{d y}{y}\left(\frac{1}{y}-\frac{1}{\operatorname{sh} y}\right)\left(1-e^{-2 y z}\right), \quad \operatorname{Re} z>0 .
$$

Now the function on the lhs is analytic in $\mathrm{C}^{-}$and the integral on the rhs converges absolutely for $\operatorname{Re} z \geqslant 0$. Thus, (A31) holds true for $\operatorname{Re} z=0$, too. Putting $z=i x$ and $z=-i x, x \in \mathbb{R}$, in (A31), and taking the sum of the resulting equations, we obtain using (A20)

$$
\ln (\pi / \operatorname{ch} \pi x)-\ln \pi+\pi x=C-\int_{0}^{\infty} \frac{d y}{y}\left(\frac{1}{y}-\frac{1}{\operatorname{sh} y}\right) \cos 2 y x,
$$

where we have set

$$
C \equiv \int_{0}^{\infty} \frac{d y}{y}\left(\frac{1}{y}-\frac{1}{\operatorname{sh} y}\right)
$$

If we now take $x \rightarrow \infty$ in (A32), then the integral has limit 0 (by virtue of the Riemann-Lebesgue lemma), so we must have

$$
C=\ln 2 \text {. }
$$

Combining this with (A31) and (A21), we obtain the integral representation

$$
\Gamma(z+1 / 2)=(2 \pi)^{1 / 2} \exp \left(z \ln z-z-\frac{1}{2} \int_{0}^{\infty} \frac{d y}{y}\left(\frac{1}{y}-\frac{1}{\operatorname{sh} y}\right) e^{-2 y z}\right),
$$

which holds true for $\operatorname{Re} z \geqslant 0$.

Next, we put $q=2, p=2 w$ in (A29) and integrate w.r.t. $w$ from 0 to $z$ to obtain the identity

$$
z \ln z-z=\int_{0}^{\infty} \frac{d y}{y}\left(e^{-2 y} z+\frac{e^{-2 y z}-1}{2 y}\right) .
$$

Inserting this in (A35), we get the representation

$$
\Gamma(z+1 / 2)=(2 \pi)^{1 / 2} \exp \left(\int_{0}^{\infty} \frac{d y}{y}\left(z e^{-2 y}-\frac{1}{2 y}+\frac{e^{-2 y z}}{2 \operatorname{shy}}\right)\right),
$$

which is valid for $\operatorname{Re} z>-1 / 2$. A routine calculation using (A37) and (A29) (with $q=2, p=2 w$ ) now yields

$$
\frac{\Gamma(w+\lambda)}{\Gamma(w+\mu)} e^{(\mu-\lambda) \ln w}=\exp \left(\int_{0}^{\infty} \frac{d t}{t} e^{-w t}\left(\lambda-\mu+\frac{e^{-\lambda t}-e^{-\mu t}}{1-e^{-t}}\right)\right),
$$

which holds true for $\operatorname{Re} w>\max (0,-\operatorname{Re} \lambda,-\operatorname{Re} \mu)$. Therefore, the function

$$
F(w, \lambda) \equiv \frac{w+\lambda}{w-\lambda}\left(\frac{\Gamma(w+\lambda)}{\Gamma(w-\lambda)} e^{-2 \lambda \ln w}\right)^{2}
$$

admits the representation 


$$
F(w, \lambda)=\exp \left(2 \int_{0}^{\infty} \frac{d t}{t} e^{-w t}(2 \lambda-\operatorname{sh} \lambda t \operatorname{cth} t / 2)\right),
$$

provided $\operatorname{Re} w>|\operatorname{Re} \lambda|$. (To check this, use (A38) and (A29) with $q=w-\lambda$ and $p=w+\lambda$.)

The function $F(w, \lambda)$ will reappear in Appendix B; it is crucial for obtaining Prop. III.5 in Subsection III A. We conclude by deriving some formulas that are used towards the end of Subsection IV A. First, (A12) entails the cosine transform

$$
\begin{aligned}
& \psi((p+1+i x) / 2)-\psi((q+1+i x) / 2)+(x \rightarrow-x) \\
& \quad=2 \int_{0}^{\infty} \frac{d y}{\operatorname{sh} y}\left(e^{-q y}-e^{-p y}\right) \cos x y, \quad \operatorname{Re} p, \operatorname{Re} q>-1, \quad x \in \mathbb{R}
\end{aligned}
$$

Now we take $\operatorname{Re} p \in(-1,1)$ and put $q=-p$. Using (A14) and the elementary identity

$$
\operatorname{tg}(\sigma+i \tau)+\operatorname{tg}(\sigma-i \tau)=\frac{2 \sin 2 \sigma}{\cos 2 \sigma+\operatorname{ch} 2 \tau}, \quad \sigma, \tau \in \mathrm{C},
$$

we obtain

$$
\int_{0}^{\infty} d y \frac{\operatorname{sh} p y}{\operatorname{sh} y} \cos x y=\frac{\pi}{2} \frac{\sin \pi p}{\cos \pi p+\operatorname{ch} \pi x}, \quad|\operatorname{Re} p|<1, \quad x \in \mathbb{R} .
$$

Integrating this with respect to $p$ from $s$ to $t$ yields

$$
2 \int_{0}^{\infty} \frac{d y}{y} \frac{(\operatorname{cht} y-\operatorname{ch} s y)}{\operatorname{sh} y} \cos x y=\ln \left(\frac{\operatorname{ch} \pi x+\cos \pi s}{\operatorname{ch} \pi x+\cos \pi t}\right), \quad|\operatorname{Re} s|,|\operatorname{Re} t|<1 .
$$

Finally, we integrate (A41) w.r.t. $x$ from 0 to $-2 i s$ and put $p=t+\lambda, q=t-\lambda$. The resulting formula entails the identity

$$
\begin{gathered}
\frac{\Gamma(s+(1+\lambda+t) / 2) \Gamma(-s+(1-\lambda+t) / 2)}{(s \rightarrow-s)}=\exp \left(2 \int_{0}^{\infty} \frac{d y}{y} \frac{\operatorname{sh} \lambda y \operatorname{sh} 2 s y}{\operatorname{sh} y} e^{-t y}\right), \\
\operatorname{Re} t-|\operatorname{Re} \lambda|>-1, \quad s \in i \mathbb{R} .
\end{gathered}
$$

\section{APPENDIX B: UNIFORM ESTIMATES}

The main goal of this appendix consists in deriving bounds that are sufficiently strong to control the convergence and meromorphy properties of infinite products involving gamma functions, which occur in the main text. Our tool for doing so is Theorem B.1, which deals with Laplace transforms $L(w), w \in \mathbb{C}$, of a certain type. More generally, this theorem can be used to obtain estimates on remainders in asymptotic expansions that hold uniformly in sectorial regions $|\operatorname{Arg} w| \leqslant \pi-\epsilon,|w| \geqslant K=K(\epsilon)$ for any $\epsilon>0$. As such, it is inspired by, but simpler than, the methods that can be found in Ref. 20, Sections 21-25, and Ref. 16, Section 13.6.

Assume $h(z)$ is a function that is analytic in the right half plane $\operatorname{Re} z>0$ and at $z=0$. Moreover, assume $h(z)$ satisfies the bound

$$
\left|h\left(t e^{i \phi}\right)\right| \leqslant C(\chi) e^{r t}, \quad \forall(t, \phi) \in[0, \infty) \times[-\chi, \chi],
$$

where $\chi \in[0, \pi / 2)$ and $r \in[0, \infty)$, and where $C(\chi)$ is a positive non-decreasing function on $[0, \pi / 2)$.

Theorem B.1: The function 


$$
L(w) \equiv \int_{0}^{\infty} e^{-w t} h(t) d t
$$

is well defined and analytic in $\{\operatorname{Re} w>r\}$. Furthermore, $L(w)$ can be continued to a function that is analytic in

$$
U_{r} \equiv\{\operatorname{Re} w \geqslant 0,|w|>r\} \cup\{\operatorname{Re} w<0,|\operatorname{Im} w|>r\} .
$$

Finally, fixing $\chi \in[0, \pi / 2)$ and $R>r$ one has

$$
|L(w)| \leqslant C(\chi)(R-r)^{-1}, \quad \forall w \in S_{R, \chi}
$$

where

$$
S_{R, \chi} \equiv \cup_{|\phi| \leqslant \chi}\left\{\operatorname{Re}\left(e^{i \phi} w\right) \geqslant R\right\} .
$$

Proof: The first assertion is obvious. To prove the second one, consider the integral

$$
e^{i \chi} \int_{0}^{\infty} \exp \left(-w t e^{i \chi}\right) h\left(t e^{i \chi}\right) d t, \quad \chi \in(-\pi / 2, \pi / 2) .
$$

Due to the bound (B1) this defines a function $L_{\chi}(w)$ that is analytic in the region

$$
U_{r, \chi} \equiv\left\{\operatorname{Re}\left(e^{i \chi} w\right)>r\right\} .
$$

We claim that $L_{\chi}(w)$ equals $L(w)$ in $U_{r, 0} \cap U_{r, \chi}$. Taking this for granted, the second assertion follows, since we have

$$
U_{r}=\cup_{|\chi|<\pi / 2} U_{r, \chi}
$$

To prove the claim we first take $\chi \in[0, \pi / 2)$. Fixing $w \in U_{r, 0} \cap U_{r, \chi}$, we then have

$$
\inf _{\phi \in[0, \chi]}\left\{\operatorname{Re}\left(e^{i \phi} w\right)\right\}=\min \left(\operatorname{Re} w, \operatorname{Re}\left(e^{i \chi_{w}}\right)\right)=r+\epsilon
$$

with $\epsilon=\epsilon(w)>0$. Using (B1) we now obtain

$$
\left|\exp \left(-w t e^{i \phi}\right) h\left(t e^{i \phi}\right)\right| \leqslant C(\chi) e^{-\epsilon t}, \quad \forall(\phi, t) \in[0, \chi] \times[0, \infty) .
$$

This bound entails that the integral of $e^{-w z} h(z)$ over the contour $z=K e^{i \phi}, \phi \in[0, \chi]$, vanishes for $K \rightarrow \infty$. Thus we may replace the contour $t e^{i \chi}, t \in[0, \infty)$, in the $z$-plane by the positive real axis, yielding $L_{\chi}(w)=L(w)$. This proves our claim for non-negative $\chi$, and the same reasoning applies to negative $\chi$.

It remains to prove (B4). To this end we fix $w \in S_{R, \chi}$. In view of (B5) we can find $\phi$ $\in[-\chi, \chi]$ such that $\operatorname{Re}\left(w e^{i \phi}\right) \geqslant R$. Then we get

$$
|L(w)|=\left|L_{\phi}(w)\right| \leqslant \int_{0}^{\infty}\left|\exp \left(-w t e^{i \phi}\right) h\left(t e^{i \phi}\right)\right| d t \leqslant C(\chi) \int_{0}^{\infty} e^{-R t} e^{r t} d t=C(\chi)(R-r)^{-1},
$$

where we used (B1). Thus (B4) holds true.

To illustrate how this result can be applied, we consider the Laplace transform 


$$
\begin{gathered}
\mathscr{L}(w) \equiv \int_{0}^{\infty} e^{-w t} f(t) d t, \\
f(t) \equiv \frac{1}{t}-\frac{1}{\operatorname{sh} t},
\end{gathered}
$$

occurring on the rhs of (A30). Integrating by parts $n$ times, we obtain

$$
\mathscr{L}(w)-\sum_{l=1}^{n} w^{-l} f^{(l-1)}(0)=w^{-n} \int_{0}^{\infty} e^{-w t} f^{(n)}(t) d t .
$$

Now the function $h(t) \equiv f^{(n)}(t)$ satisfies the assumptions of Theorem B.1 with $r=0$, so (B4) yields a bound on the remainder integral that is uniform in $S_{R, \chi}$; fixing $\delta>0$, the sectorial region $|\operatorname{Arg} w| \leqslant \pi / 2+\chi-\delta,|w| \geqslant K$, belongs to $S_{R, \chi}$ for $K=K(\delta, R, \chi)$ large enough, cf. Fig. 1 .

The Laplace transform in (A35) can be handled in the same way. This yields an asymptotic expansion that is substantially equivalent to the Stirling series, valid uniformly in sectorial regions of the above type.

For applications in the main text, however, we shall exploit Theorem B.1 to obtain uniform estimates pertaining to the Laplace transforms

$$
\mathscr{L}_{j}(w)=\int_{0}^{\infty} e^{-w t} f_{j}(t) d t, \quad j=1,2,3
$$

with

$$
\begin{gathered}
f_{1} \equiv \frac{1}{t}(2 \lambda-\operatorname{sh} \lambda t \operatorname{cth} t / 2) \Rightarrow f_{1}(0)=0, \quad f_{1}^{\prime}(0)=-\lambda\left(2 \lambda^{2}+1\right) / 6, \\
f_{2} \equiv \frac{1}{t}\left(\lambda-\mu+\frac{e^{-\lambda t}-e^{-\mu t}}{1-e^{-t}}\right) \Rightarrow f_{2}(0)=(\lambda-\mu)(\lambda+\mu-1) / 2, \\
f_{3} \equiv \frac{\operatorname{sh} \lambda t \operatorname{sh} \mu t \operatorname{sh} \kappa t}{t \operatorname{sh} t} \Rightarrow f_{3}(0)=0, \quad f_{3}^{\prime}(0)=\lambda \mu \kappa .
\end{gathered}
$$

Then the functions $h_{1} \equiv f_{1}^{\prime \prime}, h_{2} \equiv f_{2}^{\prime}$ and $h_{3} \equiv f_{3}^{\prime \prime}$ satisfy the hypotheses of Theorem B.1. Correspondingly, we deduce the bounds

$$
\begin{gathered}
\left|\mathscr{L}_{1}(w, \lambda)+\frac{\lambda\left(2 \lambda^{2}+1\right)}{6 w^{2}}\right| \leqslant \frac{C_{1}(\chi, \lambda)}{\left|w^{2}\right|\left(R-r_{1}\right)}, \quad r_{1} \equiv|\lambda|, \\
\left|\mathscr{L}_{2}(w, \lambda, \mu)-\frac{(\lambda-\mu)(\lambda+\mu+1)}{2 w}\right| \leqslant \frac{C_{2}(\chi, \lambda, \mu)}{|w|\left(R-r_{2}\right)}, \quad r_{2} \equiv \max (|\lambda|,|\mu|), \\
\left|\mathscr{L}_{3}(w, \lambda, \mu, \kappa)-\frac{\lambda \mu \kappa}{w^{2}}\right| \leqslant \frac{C_{3}(\chi, \lambda, \mu, \kappa)}{\left|w^{2}\right|\left(R-r_{3}\right)}, \quad r_{3} \equiv|\lambda|+|\mu|+|\kappa|,
\end{gathered}
$$

which hold true for $R>r_{j}$ and all $w \in S_{R, \chi}$. The functions $C_{j}$ are positive and non-decreasing in $\chi$ for fixed values of the parameters, and they are continuous in the parameters for fixed $\chi$.

Recalling (A40), one easily obtains a corresponding bound on

$$
F(w, \lambda)=\exp \left(2 \mathscr{L}_{1}(w, \lambda)\right) .
$$




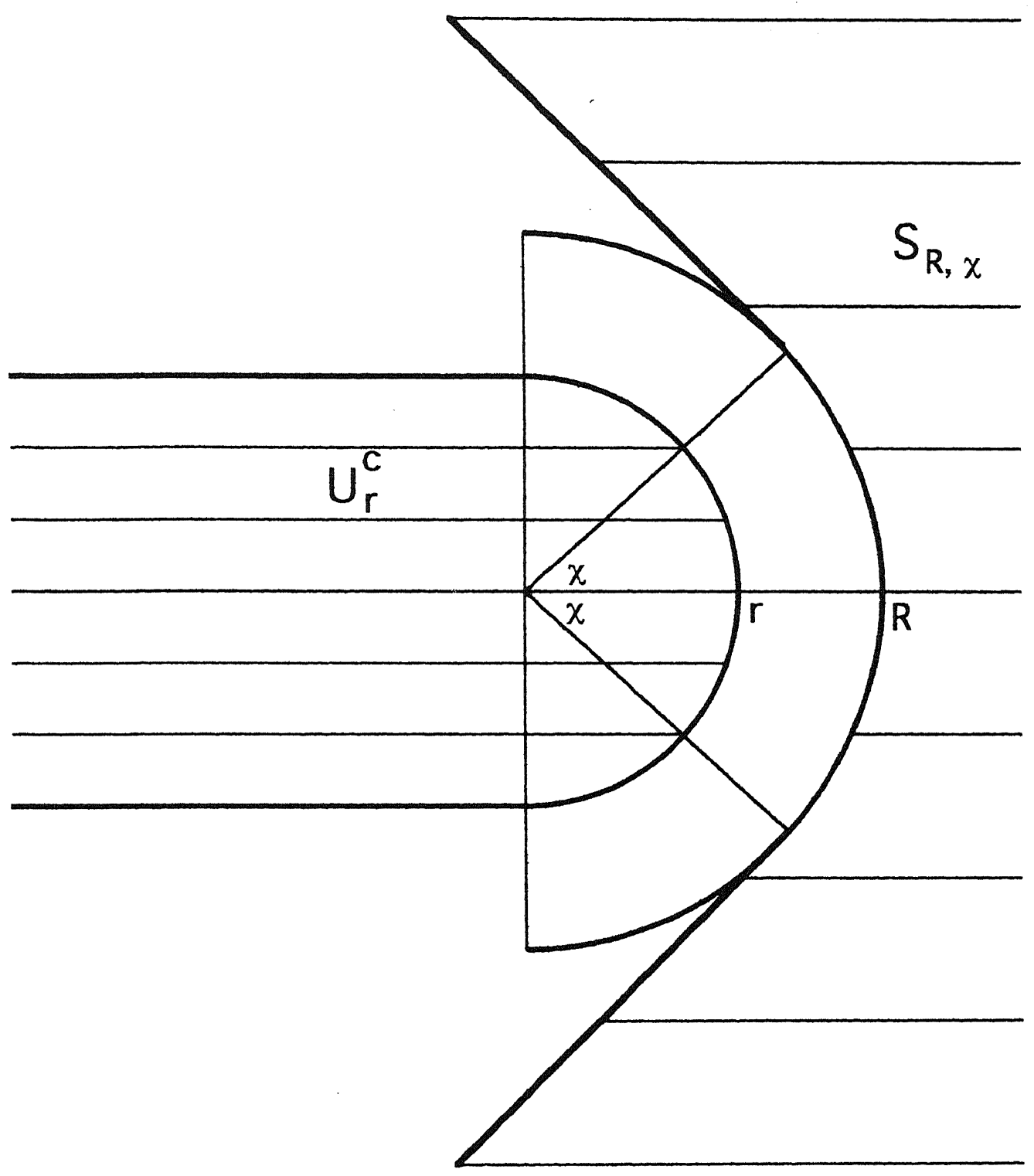

FIG. 1. The region $S_{R, \chi}$ and the complement of the region $U_{r}$.

We will need this bound in Subsection III A. Similarly, from (A38) one has

$$
\frac{\Gamma(w+\lambda)}{\Gamma(w+\mu)} e^{(\mu-\lambda) \ln w}=\exp \left(\mathscr{E}_{2}(w, \lambda, \mu)\right),
$$

and the bound on the lhs following from (B20) will be used several times in Subsection IV A. For the applications of (B19) and (B20) we do not need a bound on the parameter dependence of $C_{1}$ and $C_{2}$; continuity in the parameters suffices. As concerns (B21), however, it is important to 
have more information on $C_{3}$. Indeed, in Subsection IV A we shall use (B21) on four occasions; in two cases the parameters vary over C-compacts, but in the remaining applications one or two parameters go to infinity.

In order to control this divergence, we first note that the function

$$
h(t, p) \equiv \frac{\operatorname{sh} p t}{t}
$$

satisfies the bounds

$$
\left.\left|\partial_{t}^{j} h\left(t e^{i \phi}, p\right) \leqslant d_{j}(\chi)\right| p\right|^{j+1} \exp (|p| t), \quad \forall(t, \phi, p) \in[0, \infty) \times[-\chi, \chi] \times \mathbb{C},
$$

with $d_{j}$ positive non-decreasing functions on $[0, \pi / 2$ ), and $j=0,1,2$. (Write $h$ as $p f(p t), f(x) \equiv \operatorname{sh} x / x$, to verify this.) Factorizing $f_{3}$ accordingly, we deduce that the function $C_{3}$ in the bound (B1) on $f_{3}^{\prime \prime}$ satisfies

$$
\left|C_{3}(\chi, \lambda, \mu, \kappa)\right| \leqslant d(\chi)|\lambda \mu|\left(|\lambda|^{2}+|\mu|^{2}+|\kappa|^{2}+|\lambda \mu|+|\lambda \kappa|+|\mu \kappa|\right)
$$

with $d$ positive and non-decreasing on $[0, \pi / 2)$. This bound on the parameter dependence is sufficient for our purposes.

${ }^{1}$ S. N. M. Ruijsenaars, "Finite-dimensional soliton systems," in Integrable and superintegrable systems, edited by B. Kupershmidt (World Scientific, Singapore, 1990), pp. 165-206.

${ }^{2}$ S. N. M. Ruijsenaars, "Systems of Calogero-Moser type," to be published in Proceedings of the 1994 Banff summer school 'Particles and fields', edited by G. Semenoff and L. Vinet.

${ }^{3}$ J. Thomae, "Beiträge zur Theorie der durch die Heinesche Reihe darstellbaren Funktionen," J. Reine Angew. Math. 70, 258-281 (1869).

${ }^{4}$ G. Gasper and M. Rahman, Basic hypergeometric series (Cambridge U.P., Cambridge, 1990).

${ }^{5}$ N. E. Nörlund, Vorlesungen über Differenzenrechnung (Springer, Berlin, 1924).

${ }^{6} \mathrm{~L}$. M. Milne-Thomson, The calculus of finite differences (Macmillan, London, 1933).

${ }^{7} \mathrm{H}$. Meschkowski, Differenzengleichungen (Vandenhoeck \& Ruprecht, Göttingen, 1959).

${ }^{8}$ P. Schroth, "Zur Definition der Nörlundschen Hauptlösung von Differenzengleichungen," Manuscr. Math. 24, 239-251 (1978).

${ }^{9}$ L. Hörmander, An introduction to complex analysis in several variables (Van Nostrand, London, 1966).

${ }^{10}$ S. N. M. Ruijsenaars and H. Schneider, "A new class of integrable systems and its relation to solitons," Ann. Phys. (N.Y.) 170, 370-405 (1986).

${ }^{11}$ S. N. M. Ruijsenaars, "Complete integrability of relativistic Calogero-Moser systems and elliptic function identities," Commun. Math. Phys. 110, 191-213 (1987).

${ }^{12}$ M. A. Olshanetsky and A. M. Perelomov, "Quantum integrable systems related to Lie algebras," Phys. Reps. 94, 313-404 (1983).

${ }^{13}$ S. N. M. Ruijsenaars, "Generalized Lamé functions," to be published.

${ }^{14}$ A. B. Zamolodchikov and A. B. Zamolodchikov, "Factorized $S$-matrices in two dimensions as the exact solutions of certain relativistic quantum field theory models," Ann. Phys. (N.Y.) 120, 253-291 (1979).

${ }^{15}$ R. J. Baxter, Exactly solved models in statistical mechanics (Academic, New York, 1982).

${ }^{16}$ E. T. Whittaker and G. N. Watson, A course of modern analysis (Cambridge U.P., Cambridge, 1973).

${ }^{17}$ S. N. M. Ruijsenaars, "Action-angle maps and scattering theory for some finite-dimensional integrable systems. III. Sutherland type systems and their duals," Publ. RIMS, Kyoto Univ. 31, 247-353 (1995).

${ }^{18}$ A. Erdélyi, Tables of integral transforms, Vol. I (McGraw-Hill, New York, 1954).

${ }^{19}$ I. S. Gradshteyn and I. M. Ryzhik, Table of integrals, series, and products (Academic, New York, 1965).

${ }^{20}$ E. T. Copson, Asymptotic expansions (Cambridge U.P., Cambridge, 1965). 